\title{
Operators and higher genus mirror curves
}

\section{Santiago Codesido, ${ }^{a}$ Jie $\mathbf{G u}^{b}$ and Marcos Mariño ${ }^{a}$}

${ }^{a}$ Département de Physique Théorique et section de Mathématiques, Université de Genève, Genève, CH-1211 Switzerland

${ }^{b}$ Laboratoire de Physique Théorique de l'École Normale Supérieure, CNRS, PSL Research University,

Sorbonne Universités, UPMC, 75005 Paris, France

E-mail: santiago.codesido@unige.ch, jie.gu@lpt.ens.fr, marcos.marino@unige.ch

ABSTRACT: We perform further tests of the correspondence between spectral theory and topological strings, focusing on mirror curves of genus greater than one with nontrivial mass parameters. In particular, we analyze the geometry relevant to the $\mathrm{SU}(3)$ relativistic Toda lattice, and the resolved $\mathbb{C}^{3} / \mathbb{Z}_{6}$ orbifold. Furthermore, we give evidence that the correspondence holds for arbitrary values of the mass parameters, where the quantization problem leads to resonant states. We also explore the relation between this correspondence and cluster integrable systems.

KEYWORDs: Nonperturbative Effects, Topological Strings, Lattice Integrable Models

ARXIV EPRINT: 1609.00708 


\section{Contents}

1 Introduction 1

2 Spectral theory and topological strings 3

2.1 Mirror curves and spectral theory 3

2.2 Spectral determinants and topological strings 8

$\begin{array}{lll}2.3 & \text { Perturbed } \mathrm{O}_{m, n} \text { operators } & 13\end{array}$

3 The $Y^{3,0}$ geometry 16

$\begin{array}{ll}3.1 \text { Mirror curve and operator content } & 16\end{array}$

$\begin{array}{ll}3.2 \text { 't Hooft expansion of the fermionic traces } & 18\end{array}$

$\begin{array}{lll}3.3 & \text { Topological string calculations } & 22\end{array}$

3.4 Spectral traces from Airy functions 26

4 The resolved $\mathbb{C}^{3} / \mathbb{Z}_{6}$ orbifold $\quad 30$

4.1 Mirror curve and operator content 30

$\begin{array}{lll}4.2 & \text { Topological string calculations } & 32\end{array}$

$\begin{array}{lll}4.3 & \text { Spectral traces from Airy functions } & 35\end{array}$

$\begin{array}{lll}5 & \text { Resonances and quantum spectral curves } & 37\end{array}$

6 Quantum mirror curves and cluster integrable systems 42

7 Conclusions and discussion $\quad 46$

\section{Introduction}

Recently, a detailed, conjectural correspondence has been proposed between topological strings on toric Calabi-Yau (CY) manifolds, and the spectral theory of certain quantummechanical operators on the real line [1] (see [2] for a review). The operators arise by the quantization of the mirror curve to the toric CY, as suggested originally in [3]. This correspondence builds upon previous work on quantization and mirror symmetry [4-8], and on the exact solution of the ABJM matrix model [9-15] (reviewed in [16, 17].) It leads to exact formulae for the spectral traces and the Fredholm determinants of these operators, in terms of BPS invariants of the CY. Conversely, the genus expansion of the topological string free energy arises as an asymptotic expansion of their spectral traces, in a certain 't Hooft-like regime. In this way, the correspondence provides a non-perturbative completion of the topological string free energy. Although the general correspondence of [1] is still conjectural, it has passed many tests. In the last two years, techniques have been developed to calculate the corresponding quantities in spectral theory and shown to be in perfect agreement with the predictions of the conjecture [18-22]. Other aspects of the correspondence have been discussed in [23-26]. 
The conjectural correspondence of [1] was formulated for mirror curves of genus one. The generalization to curves of higher genus $g_{\Sigma}>1$ was proposed in [27]. In this case, the quantization of the curve leads naturally to $g_{\Sigma}$ different operators, and one can define a generalized Fredholm determinant which encodes their spectral properties. In [27], the higher genus version of the correspondence was verified in detail in the example of the resolved $\mathbb{C}^{3} / \mathbb{Z}_{5}$ orbifold, arguably the simplest toric $\mathrm{CY}$ with a genus two mirror curve. It would be desirable to have more examples and tests of the conjecture in the higher genus case, which has been comparatively less explored. The first goal of this paper is to start filling this gap by analyzing in detail two important genus two geometries. After briefly reviewing the spectral theory of the operators associated to toric CY's in section 2, we study in section 3 the CY geometry that leads to the $\mathrm{SU}(3)$ relativistic periodic Toda lattice (recently studied in [28]), and the resolved $\mathbb{C}^{3} / \mathbb{Z}_{6}$ orbifold. Both geometries have a mass parameter, which was absent in the example analyzed in [27]. Geometrically, they both engineer SU(3) super Yang-Mills theory [29], and they correspond to 5d, SU(3) gauge theories on $S^{1}$ with different values of the 5 d Chern-Simons coupling (see for example [30]). Our focus is on the spectral traces of the operators obtained by quantization of their mirror curves. In all cases, the conjectural formulae of [27] pass the tests with flying colors. Along the way, we present in section 2.3 the exact integral kernel for the inverse of a fourterm operator which is relevant for the relativistic Toda case. This generalizes the results in $[18,20]$.

The operators arising from the quantization of mirror curves depend on the so-called mass parameters of the geometry $[35,36]$. They are only compact and of trace class when these parameters satisfy some positivity conditions. This is similar to what happens in ordinary quantum mechanics. A simple example is provided by the quartic oscillator, defined by the following Schrödinger operator on $L^{2}(\mathbb{R})$ :

$$
\mathrm{H}=\frac{\mathrm{p}^{2}}{2}+\frac{\mathrm{q}^{2}}{2}+g \mathrm{q}^{4} .
$$

The inverse operator $\mathrm{H}^{-1}$ is trace class provided $g>0$ (see for example [37]). For $g<0$, the potential becomes unstable and there are no longer bound states. However, there are resonant states with a discrete set of complex eigenvalues that can be calculated by using complex dilatation techniques (see for example $[38,39]$ ). In section 5 of this paper, we point out that the spectral theory of the operators arising from mirror curves displays a very similar phenomenon. Namely, for general values of the mass parameters, these operators are no longer compact (this is easily seen by using semiclassical estimates). However, their spectral traces admit an analytic continuation to the complex planes of the mass parameters, with branch cuts, and they can be still exactly computed from topological string data by a natural extension of the conjecture in [1, 27]. In particular, we can construct an analytic continuation of the Fredholm determinant for arbitrary, complex values of the mass parameters. The vanishing locus of this analytically-continued function leads to discrete, complex values of the energy, which we interpret as resonances.

As explained in [27], since the $g_{\Sigma}$ operators arising from the mirror curve are closely related among themselves, the conjectural correspondence of [27] leads to a single quantiza- 
tion condition, given by the vanishing of the generalized Fredholm determinant. However, by a construction of Goncharov and Kenyon [31], it is possible to construct a quantum integrable system, called the cluster integrable system, for any toric $\mathrm{CY}$, leading to $g_{\Sigma}$ commuting Hamiltonians. An exact quantization condition for this integrable system was proposed in [28, 32], based on the general philosophy of [4]. The result of [32] generalizes the quantization condition of [1] in the form presented in [33]. Therefore, there are two different quantum problems arising from toric CY manifolds: on one hand we have the problem associated to the quantization of the mirror curve, which can be formulated in terms of non-commuting operators on $\mathbb{R}$. On the other hand, we have the cluster integrable system of Goncharov and Kenyon, which leads to commuting Hamiltonians on $\mathbb{R}^{g_{\Sigma}}$. In the genus one case, the two problems coincide, and in the higher genus case they should be closely related: as in the case of the standard Toda lattice, we expect that the quantization of the mirror curve leads to the quantum Baxter equation of the cluster integrable system. By requiring additional constraints on the solution of this equation, one should recover the $g_{\Sigma}$ quantization conditions. This program has not been pursued in detail. However, it was noticed in [32], in a genus two example, that an appropriate rotation of the variables in the generalized spectral determinant leads to two different functions on moduli space. The intersection of the vanishing loci of these functions turns out to coincide with the spectrum of the cluster integrable system. Recently, this observation has been generalized in [34], and there is now empirical evidence that the generalized spectral determinant of [27], after appropriate rotations of the phases of the variables, leads to at least $g_{\Sigma}$ different functions whose zero loci intersect precisely on the spectrum of the cluster integrable system. ${ }^{1}$ Another goal of this paper is to further clarify the relation between the quantization conditions of [27] and of [32]. In particular, we will show in section 5 that in some genus two geometries, reality and positivity conditions allow us to deduce the spectrum of the cluster integrable system from the generalized Fredholm determinant.

\section{Spectral theory and topological strings}

In this section, we review the construction of $g_{\Sigma}$ operators from the mirror curve of a toric $\mathrm{CY}$, their spectral theory, and the connection to the topological string theory compactified on the toric CY. We mainly follow $[1,19,20,27]$. The material on toric CYs and mirror symmetry is standard, see for example [40-43].

\subsection{Mirror curves and spectral theory}

Toric CY threefolds can be specified by a matrix of charges $Q_{i}^{\alpha}, i=0, \cdots, k+2, \alpha=$ $1, \cdots, k$ satisfying the condition,

$$
\sum_{i=0}^{k+2} Q_{i}^{\alpha}=0, \quad \alpha=1, \ldots, k .
$$

\footnotetext{
${ }^{1}$ As is obvious from this discussion, the quantization condition of [27] is more general than the one in [32], as all existing results indicate that one can recover the latter from the former.
} 
Their mirrors can be written in terms of $3+k$ complex coordinates $Y^{i} \in \mathbb{C}^{*}, i=0, \cdots, k+2$, which satisfy the constraint

$$
\sum_{i=0}^{k+2} Q_{i}^{\alpha} Y^{i}=0, \quad \alpha=1, \ldots, k .
$$

The mirror CY manifold $\widehat{X}$ is then given by

$$
w^{+} w^{-}=W_{X}
$$

where

$$
W_{X}=\sum_{i=0}^{k+2} x_{i} \mathrm{e}^{Y_{i}} .
$$

It is possible to solve the constraints (2.2), modulo a global translation, in terms of two variables which we will denote by $x, y$, and we then obtain a function $W_{X}\left(\mathrm{e}^{x}, \mathrm{e}^{y}\right)$ from (2.4). The equation

$$
W_{X}\left(\mathrm{e}^{x}, \mathrm{e}^{y}\right)=0
$$

defines a Riemann surface $\Sigma$ embedded in $\mathbb{C}^{*} \times \mathbb{C}^{*}$, which we will call the mirror curve to the toric CY threefold $X$. We note that there is a group of reparametrization symmetries of the mirror curve given by [44],

$$
\left(\begin{array}{l}
x \\
y
\end{array}\right) \rightarrow G\left(\begin{array}{l}
x \\
y
\end{array}\right), \quad G \in \operatorname{SL}(2, \mathbb{Z}) .
$$

The moduli space of the mirror curve can be parametrized by the $k+3$ coefficients $x_{i}$ of its equation (2.4), among which three can be set to 1 by the $\mathbb{C}^{*}$ scaling acting on $\mathrm{e}^{x}, \mathrm{e}^{y}$ and an overall $\mathbb{C}^{*}$ scaling. Equivalently, one can use the Batyrev coordinates

$$
z_{\alpha}=\prod_{i=0}^{k+2} x_{i}^{Q_{i}^{\alpha}}, \quad \alpha=1, \ldots, k
$$

which are invariant under the $\mathbb{C}^{*}$ actions. In order to write down the mirror curves, it is also useful to introduce a two-dimensional Newton polygon $\mathcal{N}$. The points of this polygon are given by

$$
\nu^{(i)}=\left(\nu_{1}^{(i)}, \nu_{2}^{(i)}\right)
$$

in such a way that the extended vectors

$$
\bar{\nu}^{(i)}=\left(1, \nu_{1}^{(i)}, \nu_{2}^{(i)}\right), \quad i=0, \cdots, k+2,
$$

satisfy the relations

$$
\sum_{i=0}^{k+2} Q_{i}^{\alpha} \bar{\nu}^{(i)}=0
$$


This Newton polygon is nothing else but the support of the $3 \mathrm{~d}$ toric fan of the toric CalabYau threefold on a hyperplane located at $(1, *, *)$. The function on the l.h.s. of $(2.5)$ is then given by the Newton polynomial of the polygon $\mathcal{N}$,

$$
W_{X}\left(\mathrm{e}^{x}, \mathrm{e}^{y}\right)=\sum_{i=0}^{k+2} x_{i} \exp \left(\nu_{1}^{(i)} x+\nu_{2}^{(i)} y\right) .
$$

Clearly, there are many sets of vectors satisfying the relations (2.10), but they lead to curves differing in a reparametrization or a global translation, which are therefore equivalent. It can be seen that the genus of $\Sigma, g_{\Sigma}$, is given by the number of inner points of $\mathcal{N}$. We also notice that among the coefficients $x_{i}$ of the mirror curve, $g_{\Sigma}$ of them are "true" moduli of the geometry, corresponding to the inner points of $\mathcal{N}$, while $r_{\Sigma}$ of them are the socalled "mass parameters", corresponding to the points on the boundary of the polygon (this distinction has been emphasized in $[35,36]$ ). In order to distinguish them, we will denote the former by $\kappa_{i}, i=1, \ldots, g_{\Sigma}$ and the latter by $\xi_{j}, j=1, \ldots, r_{\Sigma}$. It is obvious that we can translate the Newton polygon in such a way that the inner point associated to a given $\kappa_{i}$ is the origin. In this way we obtain what we will call the canonical forms of the mirror curve

$$
\mathcal{O}_{i}(x, y)+\kappa_{i}=0, \quad i=1, \cdots, g_{\Sigma},
$$

where $\mathcal{O}_{i}(x, y)$ is a polynomial in $\mathrm{e}^{x}$, $\mathrm{e}^{y}$. Note that, for $g_{\Sigma}=1$, there is a single canonical form. Different canonical forms are related by reparametrizations of the form (2.6) and by overall translations, which lead to overall monomials, so we will write

$$
\mathcal{O}_{i}+\kappa_{i}=\mathcal{P}_{i j}\left(\mathcal{O}_{j}+\kappa_{j}\right), \quad i, j=1, \cdots, g_{\Sigma}
$$

where $\mathcal{P}_{i j}$ is of the form $\mathrm{e}^{\lambda x+\mu y}, \lambda, \mu \in \mathbb{Z}$. Equivalently, we can write

$$
\mathcal{O}_{i}=\mathcal{O}_{i}^{(0)}+\sum_{j \neq i} \kappa_{j} \mathcal{P}_{i j}
$$

The functions $\mathcal{O}_{i}(x, y)$ appearing in the canonical forms of the mirror curves can be quantized $[1,27]$. To do this, we simply promote the variables $x, y$ to Heisenberg operators $\mathrm{x}, \mathrm{y}$ satisfying

$$
[\mathrm{x}, \mathrm{y}]=\mathrm{i} \hbar,
$$

and we use the Weyl quantization prescription. In this way we obtain $g_{\Sigma}$ different operators, which we will denote by $\mathrm{O}_{i}, i=1, \cdots, g_{\Sigma}$. These operators are self-adjoint. The equation of the mirror curve itself is promoted to an operator

$$
\mathrm{W}_{X, i} \equiv \mathrm{O}_{i}+\kappa_{i},
$$

which we call the quantum mirror curve. Different canonical forms are related by the quantum version of (2.13),

$$
\mathrm{O}_{i}+\kappa_{i}=\mathrm{P}_{i j}^{1 / 2}\left(\mathrm{O}_{j}+\kappa_{j}\right) \mathrm{P}_{i j}^{1 / 2}, \quad i, j=1, \cdots, g_{\Sigma},
$$


where $\mathrm{P}_{i j}$ is the operator corresponding to the monomial $\mathcal{P}_{i j}$. We will also denote by $\mathrm{O}_{i}^{(0)}$ the operator associated to the function $\mathcal{O}_{i}^{(0)}$ in $(2.14)$. This can be regarded as an "unperturbed" operator, while the moduli $\kappa_{j}$ encode different perturbations of it. We also define the inverse operators,

$$
\rho_{i}=\mathrm{O}_{i}^{-1}, \quad \rho_{i}^{(0)}=\left(\mathrm{O}_{i}^{(0)}\right)^{-1}, \quad i=1, \cdots, g_{\Sigma} .
$$

It is easy to see that [27]

$$
\mathrm{P}_{i j}=\mathrm{P}_{j i}^{-1}, \quad i \neq j,
$$

and

$$
\mathrm{P}_{i k}=\mathrm{P}_{i j}^{1 / 2} \mathrm{P}_{j k} \mathrm{P}_{i j}^{1 / 2}, \quad i \neq k .
$$

We want to study now the spectral theory of the operators $\mathrm{O}_{i}, i=1, \cdots, g_{\Sigma}$. The appropriate object to consider turns out to be the generalized spectral determinant introduced in [27]. Let us consider the following operators,

$$
\mathrm{A}_{j l}=\rho_{j}^{(0)} \mathrm{P}_{j l}, \quad j, l=1, \cdots, g_{\Sigma} .
$$

Let us suppose that these operators are of trace class (this turns out to be the case in all known examples, provided some positivity conditions on the mass parameters are satisfied). Then, the generalized spectral determinant associated to the CY $X$ is given by

$$
\Xi_{X}(\kappa ; \hbar)=\operatorname{det}\left(1+\kappa_{1} \mathrm{~A}_{j 1}+\cdots+\kappa_{g_{\Sigma}} \mathrm{A}_{j g_{\Sigma}}\right) .
$$

Due to the trace class property of the operators $\mathrm{A}_{j l}$, this quantity is well-defined, and its definition does not depend on the choice of the index $j$, due to the similarity transformation

$$
\mathrm{A}_{i l}=\mathrm{P}_{i j}^{-1 / 2} \mathrm{~A}_{j l} \mathrm{P}_{i j}^{1 / 2} .
$$

As shown in [45], (2.22) is an entire function of the moduli $\kappa_{1}, \cdots, \kappa_{g_{\Sigma}}$. In particular, it can be expanded around the origin $\boldsymbol{\kappa}=0$, as follows,

$$
\Xi_{X}(\boldsymbol{\kappa} ; \hbar)=\sum_{N_{1} \geq 0} \cdots \sum_{N_{g_{\Sigma}} \geq 0} Z_{X}(\boldsymbol{N} ; \hbar) \kappa_{1}^{N_{1}} \cdots \kappa_{g_{\Sigma}}^{N_{g_{\Sigma}}}
$$

with the convention that

$$
Z_{X}(0, \cdots, 0 ; \hbar)=1
$$

This expansion defines the (generalized) fermionic spectral traces $Z_{X}(N ; \hbar)$ of the toric CY $X$. Both $\Xi_{X}(\boldsymbol{\kappa} ; \hbar)$ and $Z_{X}(\boldsymbol{N} ; \hbar)$ depend in addition on the mass parameters, gathered in a vector $\boldsymbol{\xi}$. When needed, we will indicate this dependence explicitly and write $\Xi_{X}(\boldsymbol{\kappa} ; \boldsymbol{\xi}, \hbar)$, $Z_{X}(\boldsymbol{N} ; \boldsymbol{\xi}, \hbar)$. As shown in [27], one can use classical results in Fredholm theory to obtain determinant expressions for these fermionic traces. Let us consider the kernels $A_{j l}\left(x_{m}, x_{n}\right)$ of the operators defined in (2.21), and let us construct the following matrix:

$$
R_{j}\left(x_{m}, x_{n}\right)=A_{j l}\left(x_{m}, x_{n}\right) \quad \text { if } \quad \sum_{s=1}^{l-1} N_{s}<m \leq \sum_{s=1}^{l} N_{s} .
$$


Then, we have that ${ }^{2}$

$$
Z_{X}(\boldsymbol{N} ; \hbar)=\frac{1}{N_{1} ! \cdots N_{g_{\Sigma}} !} \int \operatorname{det}_{m, n}\left(R_{j}\left(x_{m}, x_{n}\right)\right) \mathrm{d}^{N} x,
$$

where

$$
N=\sum_{s=1}^{g_{\Sigma}} N_{s}
$$

In the case $g_{\Sigma}=2$, this formula becomes

$$
Z_{X}\left(N_{1}, N_{2} ; \hbar\right)=\frac{1}{N_{1} ! N_{2} !} \int \operatorname{det}\left(\begin{array}{ccc}
A_{j 1}\left(x_{1}, x_{1}\right) & \cdots & A_{j 1}\left(x_{1}, x_{N}\right) \\
\vdots & & \vdots \\
A_{j 1}\left(x_{N_{1}}, x_{1}\right) & \cdots & A_{j 1}\left(x_{N_{1}}, x_{N}\right) \\
A_{j 2}\left(x_{N_{1}+1}, x_{1}\right) & \cdots & A_{j 2}\left(x_{N_{1}+1}, x_{N}\right) \\
\vdots & & \vdots \\
A_{j 2}\left(x_{N}, x_{1}\right) & \cdots & A_{j 2}\left(x_{N}, x_{N}\right)
\end{array}\right) \mathrm{d} x_{1} \cdots \mathrm{d} x_{N} .
$$

One finds, for example

$$
\begin{aligned}
Z_{X}(1,1 ; \hbar) & =\operatorname{Tr} \mathrm{A}_{j 1} \operatorname{Tr} \mathrm{A}_{j 2}-\operatorname{Tr}\left(\mathrm{A}_{j 1} \mathrm{~A}_{j 2}\right) \\
& =\int \mathrm{d} x_{1} \mathrm{~d} x_{2}\left(A_{j 1}\left(x_{1}, x_{1}\right) A_{j 2}\left(x_{2}, x_{2}\right)-A_{j 1}\left(x_{1}, x_{2}\right) A_{j 2}\left(x_{2}, x_{1}\right)\right),
\end{aligned}
$$

as well as

$$
\begin{aligned}
& Z_{X}(2,1 ; \hbar)=\operatorname{Tr}\left(\mathrm{A}_{j 1}^{2} \mathrm{~A}_{j 2}\right)-\frac{1}{2} \operatorname{Tr}\left(\mathrm{A}_{j 1}^{2}\right) \operatorname{Tr} \mathrm{A}_{j 2}+\frac{1}{2}\left(\operatorname{Tr} \mathrm{A}_{j 1}\right)^{2} \operatorname{Tr} \mathrm{A}_{j 2}-\operatorname{Tr} \mathrm{A}_{j 1} \operatorname{Tr}\left(\mathrm{A}_{j 1} \mathrm{~A}_{j 2}\right) \\
& Z_{X}(1,2 ; \hbar)=\operatorname{Tr}\left(\mathrm{A}_{j 1} \mathrm{~A}_{j 2}^{2}\right)-\frac{1}{2} \operatorname{Tr} \mathrm{A}_{j 1} \operatorname{Tr}\left(\mathrm{A}_{j 2}^{2}\right)+\frac{1}{2} \operatorname{Tr} \mathrm{A}_{j 1}\left(\operatorname{Tr} \mathrm{A}_{j 2}\right)^{2}-\operatorname{Tr}\left(\mathrm{A}_{j 1} \mathrm{~A}_{j 2}\right) \operatorname{Tr} \mathrm{A}_{j 2}
\end{aligned}
$$

The generalized spectral determinant encodes the spectral properties of all the operators $\mathrm{O}_{i}$ in a single strike. Indeed, one has [27],

$$
\operatorname{det}\left(1+\kappa_{i} \rho_{i}\right)=\frac{\Xi_{X}(\kappa ; \hbar)}{\Xi_{X}\left(\kappa_{1}, \cdots, \kappa_{i-1}, 0, \kappa_{i+1}, \cdots, \kappa_{g_{\Sigma}} ; \hbar\right)}, \quad i=1, \cdots, g_{\Sigma} .
$$

In addition,

$$
\operatorname{det}\left(1+\kappa_{i} \rho_{i}^{(0)}\right)=\Xi_{X}\left(0, \cdots, 0, \kappa_{i}, 0, \cdots, 0 ; \hbar\right), \quad i=1, \cdots, g_{\Sigma},
$$

i.e. the generalized spectral determinant specializes to the spectral determinants of the unperturbed operators appearing in the different canonical forms of the mirror curve.

The standard spectral determinant of a single trace-class operator determines the spectrum of eigenvalues, through its zeros. The generalized spectral determinant (2.22) vanishes in a codimension one submanifold of the moduli space. It follows from (2.32) that this submanifold contains all the information about the spectrum of the operators $\rho_{i}$ appearing in the quantization of the mirror curve, as a function of the moduli $\kappa_{j}, j \neq i$.

\footnotetext{
${ }^{2}$ The determinant of the matrix $R_{j\left(x_{m}, x_{n}\right)}$ is independent of the label $j$, just like the Fredholm determinant.
} 


\subsection{Spectral determinants and topological strings}

The main conjectural result of $[1,27]$ is an explicit formula expressing the generalized spectral determinant and spectral traces in terms of of enumerative invariants of the CY $X$. To state this result, we need some basic geometric ingredients. As discussed in the previous section, the CY $X$ has $g_{\Sigma}$ moduli denoted by $\kappa_{i}, i=1, \cdots, g_{\Sigma}$. We will introduce the associated "chemical potentials" $\mu_{i}$ by

$$
\kappa_{i}=\mathrm{e}^{\mu_{i}}, \quad i=1, \cdots, g_{\Sigma} .
$$

The CY $X$ also has $r_{\Sigma}$ mass parameters, $\xi_{j}, j=1, \cdots, r_{\Sigma}$. Let $n_{\Sigma} \equiv g_{\Sigma}+r_{\Sigma}$. The Batyrev coordinates $z_{i}$ introduced in (2.7) can be written as

$$
-\log z_{i}=\sum_{j=1}^{g_{\Sigma}} C_{i j} \mu_{j}+\sum_{k=1}^{r_{\Sigma}} \alpha_{i k} \log \xi_{k}, \quad i=1, \cdots, n_{\Sigma}
$$

The coefficients $C_{i j}$ determine a $n_{\Sigma} \times g_{\Sigma}$ matrix which can be read off from the toric data of $X$. One can choose the Batyrev coordinates in such a way that, for $i=1, \cdots, g_{\Sigma}$, the $z_{i}$ 's correspond to true moduli, while for $i=g_{\Sigma}+1, \cdots, g_{\Sigma}+r_{\Sigma}$, they correspond to mass parameters. For such a choice, the non-vanishing coefficients

$$
C_{i j}, \quad i, j=1, \cdots, g_{\Sigma}
$$

form an invertible matrix, which agrees (up to an overall sign) with the charge matrix $C_{i j}$ appearing in [46]. We also recall that the standard mirror map expresses the Kähler moduli $t_{i}$ of the $\mathrm{CY}$ in terms of the Batyrev coordinates $z_{i}$

$$
-t_{i}=\log z_{i}+\tilde{\Pi}_{i}(\boldsymbol{z}), \quad i=1 \ldots, n_{\Sigma},
$$

where $\tilde{\Pi}_{i}(\boldsymbol{z})$ is a power series in $z_{i}$ with finite radius of convergence. Together with (2.35), this implies that

$$
t_{i}=\sum_{j=1}^{g_{\Sigma}} C_{i j} \mu_{j}+\sum_{k=1}^{r_{\Sigma}} \alpha_{i k} \log \xi_{k}+\mathcal{O}\left(\mathrm{e}^{-\mu}\right) .
$$

By using the quantized mirror curve, one can promote the classical mirror map to a quantum mirror map $t_{i}(\hbar)$ depending on $\hbar[6]$ (see $[36,47]$ for examples.)

$$
-t_{i}(\hbar)=\log z_{i}+\tilde{\Pi}_{i}(\boldsymbol{z} ; \hbar), \quad i=1 \ldots, n_{\Sigma} .
$$

This quantum mirror map will play an important rôle in our construction. In addition to the quantum mirror map, we need the following enumerative ingredients. First of all, we need the conventional genus $g$ free energies $F_{g}(\mathbf{t})$ of $X, g \geq 0$, in the so-called large radius frame (LRF), which encode the information about the Gromov-Witten invariants of 
$X$. They have the structure ${ }^{3}$

$$
\begin{aligned}
& F_{0}(\mathbf{t})=\frac{1}{6} \sum_{i, j, k=1}^{n_{\Sigma}} a_{i j k} t_{i} t_{j} t_{k}+4 \pi^{2} \sum_{i=1}^{n_{\Sigma}} b_{i}^{\mathrm{NS}} t_{i}+\sum_{\mathbf{d}} N_{0}^{\mathbf{d}} \mathrm{e}^{-\mathbf{d} \cdot \mathbf{t}}, \\
& F_{1}(\mathbf{t})=\sum_{i=1}^{n_{\Sigma}} b_{i} t_{i}+\sum_{\mathbf{d}} N_{1}^{\mathbf{d}} \mathrm{e}^{-\mathbf{d} \cdot \mathbf{t}}, \\
& F_{g}(\mathbf{t})=C_{g}+\sum_{\mathbf{d}} N_{g}^{\mathbf{d}} \mathrm{e}^{-\mathbf{d} \cdot \mathbf{t}}, \quad g \geq 2 .
\end{aligned}
$$

In these formulae, $N_{g}^{\mathbf{d}}$ are the Gromov-Witten invariants of $X$ at genus $g$ and multi-degree d. The coefficients $a_{i j k}, b_{i}$ are cubic and linear couplings characterizing the perturbative genus zero and genus one free energies, while $C_{g}$ is the so-called constant map contribution [48]. The constants $b_{i}^{\mathrm{NS}}$, which can be obtained from the refined holomorphic anomaly equation $[49,50]$, usually appear in the linear term of $F^{\mathrm{NS}}(\mathbf{t}, \hbar)$ (see below, $(2.47)$ ). The total free energy of the topological string is the formal series,

$$
F^{\mathrm{WS}}\left(\mathbf{t}, g_{s}\right)=\sum_{g \geq 0} g_{s}^{2 g-2} F_{g}(\mathbf{t})=F^{(\mathrm{p})}\left(\mathbf{t}, g_{s}\right)+\sum_{g \geq 0} \sum_{\mathbf{d}} N_{g}^{\mathbf{d}} \mathrm{e}^{-\mathbf{d} \cdot \mathbf{t}} g_{s}^{2 g-2},
$$

where

$$
F^{(\mathrm{p})}\left(\mathbf{t}, g_{s}\right)=\frac{1}{6 g_{s}^{2}} \sum_{i, j, k=1}^{n_{\Sigma}} a_{i j k} t_{i} t_{j} t_{k}+\sum_{i=1}^{n_{\Sigma}}\left(b_{i}+\frac{4 \pi^{2}}{g_{s}^{2}} b_{i}^{\mathrm{NS}}\right) t_{i}+\sum_{g \geq 2} C_{g} g_{s}^{2 g-2}
$$

and $g_{s}$ is the topological string coupling constant.

As found in [51], the sum over Gromov-Witten invariants in (2.41) can be resummed order by order in $\exp \left(-t_{i}\right)$, at all orders in $g_{s}$. This resummation involves the GopakumarVafa $(\mathrm{GV})$ invariants $n_{g}^{\mathbf{d}}$ of $X$, and it has the structure

$$
F^{\mathrm{GV}}\left(\mathbf{t}, g_{s}\right)=\sum_{g \geq 0} \sum_{\mathbf{d}} \sum_{w=1}^{\infty} \frac{1}{w} n_{g}^{\mathbf{d}}\left(2 \sin \frac{w g_{s}}{2}\right)^{2 g-2} \mathrm{e}^{-w \mathbf{d} \cdot \mathbf{t}} .
$$

Note that, as formal power series, we have

$$
F^{\mathrm{WS}}\left(\mathbf{t}, g_{s}\right)=F^{(\mathrm{p})}\left(\mathbf{t}, g_{s}\right)+F^{\mathrm{GV}}\left(\mathbf{t}, g_{s}\right) .
$$

In the case of toric CYs, the Gopakumar-Vafa invariants are special cases of the refined BPS invariants [52-54]. These refined invariants depend on the degrees $\mathbf{d}$ and on two non-negative half-integers or "spins", $j_{L}, j_{R}$. We will denote them by $N_{j_{L}, j_{R}}^{\mathbf{d}}$. We now define the NS free energy as

$$
F_{\mathrm{NS}}(\mathbf{t}, \hbar)=F_{\mathrm{NS}}^{\mathrm{pert}}(\mathbf{t}, \hbar)+F_{\mathrm{NS}}^{\mathrm{inst}}(\mathbf{t}, \hbar),
$$

\footnotetext{
${ }^{3}$ The formula of $F_{0}(\mathbf{t})$ differs from the one in the topological string literature by the linear term, which usually doesn't play a role in non-compact CY models. The addition of this term makes the formulae in the rest of the paper more compact.
} 
where

$$
F_{\mathrm{NS}}^{\mathrm{pert}}(\mathbf{t}, \hbar)=\frac{1}{6 \hbar} \sum_{i, j, k=1}^{n_{\Sigma}} a_{i j k} t_{i} t_{j} t_{k}+\left(\hbar+\frac{4 \pi^{2}}{\hbar}\right) \sum_{i=1}^{n_{\Sigma}} b_{i}^{\mathrm{NS}} t_{i},
$$

and

$$
F_{\mathrm{NS}}^{\mathrm{inst}}(\mathbf{t}, \hbar)=\sum_{j_{L}, j_{R}} \sum_{w, \mathbf{d}} N_{j_{L}, j_{R}}^{\mathbf{d}} \frac{\sin \frac{\hbar w}{2}\left(2 j_{L}+1\right) \sin \frac{\hbar w}{2}\left(2 j_{R}+1\right)}{2 w^{2} \sin ^{3} \frac{\hbar w}{2}} \mathrm{e}^{-w \mathbf{d} \cdot \mathbf{t}} .
$$

In this equation, the coefficients $a_{i j k}$ are the same ones that appear in (2.40). By expanding (2.45) in powers of $\hbar$, we find the NS free energies at order $n$,

$$
F^{\mathrm{NS}}(\mathbf{t}, \hbar)=\sum_{n=0}^{\infty} F_{n}^{\mathrm{NS}}(\mathbf{t}) \hbar^{2 n-1}
$$

The first term in this series, $F_{0}^{\mathrm{NS}}(\mathbf{t})$, is equal to $F_{0}(\mathbf{t})$, the standard genus zero free energy.

Following [15], we now define the modified grand potential of the CY $X$. It is the sum of two functions. The first one is

$$
\begin{aligned}
\mathrm{J}_{X}^{\mathrm{WKB}}(\boldsymbol{\mu}, \boldsymbol{\xi}, \hbar)= & \sum_{i=1}^{n_{\Sigma}} \frac{t_{i}(\hbar)}{2 \pi} \frac{\partial F^{\mathrm{NS}}(\mathbf{t}(\hbar), \hbar)}{\partial t_{i}}+\frac{\hbar^{2}}{2 \pi} \frac{\partial}{\partial \hbar}\left(\frac{F^{\mathrm{NS}}(\mathbf{t}(\hbar), \hbar)}{\hbar}\right) \\
& +\frac{2 \pi}{\hbar} \sum_{i=1}^{n_{\Sigma}}\left(b_{i}+b_{i}^{\mathrm{NS}}\right) t_{i}(\hbar)+A(\boldsymbol{\xi}, \hbar) .
\end{aligned}
$$

We note that the function $A(\boldsymbol{\xi}, \hbar)$ is only known in a closed form in some simple geometries. The second function is the "worldsheet" modified grand potential, which is obtained from the generating functional (2.43),

$$
\mathrm{J}_{X}^{\mathrm{WS}}(\boldsymbol{\mu}, \boldsymbol{\xi}, \hbar)=F^{\mathrm{GV}}\left(\frac{2 \pi}{\hbar} \mathbf{t}(\hbar)+\pi \mathrm{iB}, \frac{4 \pi^{2}}{\hbar}\right) .
$$

It involves a constant integer vector B (or "B-field") which depends on the geometry under consideration. This vector satisfies the following requirement: for all $\mathbf{d}, j_{L}$ and $j_{R}$ such that $N_{j_{L}, j_{R}}^{\mathbf{d}}$ is non-vanishing, we must have

$$
(-1)^{2 j_{L}+2 j_{R}+1}=(-1)^{\mathbf{B} \cdot \mathbf{d}} .
$$

We note that the characterization above only defines $\mathbf{B}$ up to $(2 \mathbb{Z})^{n_{\Sigma}}$. A difference choice of $\mathbf{B}$ does not change $\mathrm{J}_{X}^{\mathrm{WS}}(\boldsymbol{\mu}, \boldsymbol{\xi}, \hbar)$. The total, modified grand potential is the sum of the above two functions,

$$
\mathrm{J}_{X}(\boldsymbol{\mu}, \boldsymbol{\xi}, \hbar)=\mathrm{J}_{X}^{\mathrm{WKB}}(\boldsymbol{\mu}, \boldsymbol{\xi}, \hbar)+\mathrm{J}_{X}^{\mathrm{WS}}(\boldsymbol{\mu}, \boldsymbol{\xi}, \hbar)
$$

and it was introduced in [15]. Note that if we define

$$
F_{\mathrm{top}}\left(\mathbf{t}, g_{s}\right)=\frac{1}{6 g_{s}^{2}} \sum_{i, j, k=1}^{n_{\Sigma}} a_{i j k} t_{i} t_{j} t_{k}+\sum_{i=1}^{n_{\Sigma}}\left(b_{i}+\frac{4 \pi^{2}}{g_{s}^{2}} b_{i}^{\mathrm{NS}}\right) t_{i}+F^{\mathrm{GV}}\left(\mathbf{t}, g_{s}\right)
$$


which differs from $F\left(\mathbf{t}, g_{s}\right)$ by the terms proportional to $C_{g}, \mathbf{J}_{X}(\boldsymbol{\mu}, \boldsymbol{\xi}, \hbar)$ can be written in a slightly more compact form, i.e. ${ }^{4}$

$$
\begin{aligned}
J(\boldsymbol{\mu}, \boldsymbol{\xi}, \hbar)=\sum_{i=1}^{n_{\Sigma}} & \frac{t_{i}}{2 \pi} \frac{\partial}{\partial t_{i}} F_{\mathrm{NS}}^{\text {inst }}(\mathbf{t}(\hbar), \hbar)+\frac{\hbar^{2}}{2 \pi} \frac{\partial}{\partial \hbar}\left(\frac{F_{\mathrm{NS}}^{\text {inst }}(\mathbf{t}(\hbar), \hbar)}{\hbar}\right)+A(\boldsymbol{\xi}, \hbar) \\
& +\widehat{F}_{\text {top }}\left(\frac{2 \pi}{\hbar} \mathbf{t}(\hbar), \frac{4 \pi^{2}}{\hbar}\right)
\end{aligned}
$$

where all but the constant $A(\boldsymbol{\xi}, \hbar)$ are hidden in (refined) topological string free energies. Here we introduce the notation $\widehat{f}(\mathbf{t})$, meaning that $\mathbf{t}$ is shifted by $\pi \mathbf{i} \mathbf{B}$ in the terms of order $\exp \left(-t_{i}\right)$ (the instanton contributions). In particular,

$$
\widehat{F}_{\text {top }}\left(\mathbf{t}, g_{s}\right)=\frac{1}{6 g_{s}^{2}} \sum_{i, j, k=1}^{n_{\Sigma}} a_{i j k} t_{i} t_{j} t_{k}+\sum_{i=1}^{n_{\Sigma}}\left(b_{i}+\frac{4 \pi^{2}}{g_{s}^{2}} b_{i}^{\mathrm{NS}}\right) t_{i}+F^{\mathrm{GV}}\left(\mathbf{t}+\pi \mathbf{i} \mathbf{B}, g_{s}\right) \text {. }
$$

According to the conjecture in $[1,27]$, the spectral determinant $(2.22)$ is given by

$$
\Xi_{X}(\boldsymbol{\kappa} ; \hbar)=\sum_{\mathbf{n} \in \mathbb{Z}^{g_{\Sigma}}} \exp \left(\mathrm{J}_{X}(\boldsymbol{\mu}+2 \pi \mathbf{i n}, \boldsymbol{\xi}, \hbar)\right) .
$$

The right hand side of (2.56) defines a quantum-deformed (or generalized) Riemann theta function by

$$
\Xi_{X}(\boldsymbol{\kappa} ; \hbar)=\exp \left(\mathrm{J}_{X}(\boldsymbol{\mu}, \boldsymbol{\xi}, \hbar)\right) \Theta_{X}(\boldsymbol{\kappa} ; \hbar) .
$$

The r.h.s. of (2.56) can be computed as an expansion around the large radius point of moduli space. In the so-called "maximally supersymmetric case" $\hbar=2 \pi$, it can be written down in closed form in terms of a conventional theta function. There is an equivalent form of the conjecture which gives an integral formula for the fermionic spectral traces:

$$
Z_{X}(\boldsymbol{N} ; \hbar)=\frac{1}{(2 \pi \mathrm{i})^{g_{\Sigma}}} \int_{-\mathrm{i} \infty}^{\mathrm{i} \infty} \mathrm{d} \mu_{1} \cdots \int_{-\mathrm{i} \infty}^{\mathrm{i} \infty} \mathrm{d} \mu_{g_{\Sigma}} \exp \left\{\mathrm{J}_{X}(\boldsymbol{\mu}, \boldsymbol{\xi}, \hbar)-\sum_{i=1}^{g_{\Sigma}} N_{i} \mu_{i}\right\} .
$$

In practice, the contour integration along the imaginary axis can be deformed to a contour where the integral is convergent. For example, in the genus one case the integration contour is the one defining the Airy function (see $[1,14]$ ).

An important consequence of the representation (2.58) is the existence of a 't Hooft-like limit in which one can extract the genus expansion of the conventional topological string. The 't Hooft limit is defined by

$$
\hbar \rightarrow \infty, \quad N_{i} \rightarrow \infty, \quad \frac{N_{i}}{\hbar}=\lambda_{i} \text { fixed, } \quad i=1, \cdots, g_{\Sigma} .
$$

In this 't Hooft limit, the integral in (2.58) can be evaluated in the saddle-point approximation, and in order to have a non-trivial result, we have to consider the modified grand potential in the limit

$$
\hbar \rightarrow \infty, \quad \mu_{i} \rightarrow \infty, \quad \frac{\mu_{i}}{\hbar}=\zeta_{i} \text { fixed, } \quad i=1, \cdots, g_{\Sigma} .
$$

\footnotetext{
${ }^{4}$ Note that $F_{\mathrm{top}}\left(\mathbf{t}, g_{s}\right)$ is $F^{\mathrm{WS}}\left(\mathbf{t}, g_{s}\right)$ with the contributions from the constant maps removed. The latter can be understood as absorbed in $A(\boldsymbol{\xi}, \hbar)$.
} 
In this limit, the quantum mirror map appearing in the modified grand potential becomes trivial. We will assume that the mass parameters $\boldsymbol{\xi}$ scale in such a way that

$$
\log \hat{\xi}_{j}=\frac{2 \pi}{\hbar} \log \xi_{j}, \quad j=1, \cdots, r_{\Sigma}
$$

are fixed as $\hbar \rightarrow \infty$. In the regime (2.60), the modified grand potential has an asymptotic genus expansion of the form,

$$
\mathrm{J}_{X}^{\text {t Hooft }}(\boldsymbol{\zeta}, \hat{\boldsymbol{\xi}}, \hbar)=\sum_{g=0}^{\infty} \mathrm{J}_{g}^{X}(\boldsymbol{\zeta}, \hat{\boldsymbol{\xi}}) \hbar^{2-2 g}
$$

where

$$
\begin{aligned}
\mathrm{J}_{0}^{X}(\boldsymbol{\zeta}, \hat{\boldsymbol{\xi}}) & =\frac{1}{16 \pi^{4}} \widehat{F}_{0}(\mathbf{T})+A_{0}(\hat{\boldsymbol{\xi}}) \\
\mathrm{J}_{1}^{X}(\boldsymbol{\zeta}, \hat{\boldsymbol{\xi}}) & =A_{1}(\hat{\boldsymbol{\xi}})+\widehat{F}_{1}(\mathbf{T}), \\
\mathrm{J}_{g}^{X}(\boldsymbol{\zeta}, \hat{\boldsymbol{\xi}}) & =A_{g}(\hat{\boldsymbol{\xi}})+\left(4 \pi^{2}\right)^{2 g-2}\left(\widehat{F}_{g}(\mathbf{T})-C_{g}\right), \quad g \geq 2 .
\end{aligned}
$$

In these equations, we have introduced the rescaled Kähler parameter

$$
\mathbf{T}=\frac{2 \pi}{\hbar} \mathbf{t}
$$

The arguments $\boldsymbol{\zeta}$ and $\hat{\boldsymbol{\xi}}$ of the modified grand potential are related to the rescaled Kähler parameters $\mathbf{T}$ by

$$
T_{i}-\sum_{j=1}^{r_{\Sigma}} \alpha_{i j} \log \hat{\xi}_{j}=2 \pi \sum_{j=1}^{g_{\Sigma}} C_{i j} \zeta_{j}, \quad i=1, \cdots, g_{\Sigma}+r_{\Sigma}
$$

We have assumed that the function $A(\boldsymbol{\xi}, \hbar)$ has the expansion

$$
A(\boldsymbol{\xi}, \hbar)=\sum_{g=0}^{\infty} A_{g}(\hat{\boldsymbol{\xi}}) \hbar^{2-2 g} .
$$

The saddle point of the integral (2.58) as $\hbar \rightarrow \infty$ is then given by

$$
\lambda_{i}=\sum_{j=1}^{n_{\Sigma}} \frac{C_{j i}}{8 \pi^{3}}\left(\frac{\partial \widehat{F}_{0}}{\partial T_{j}}+4 \pi^{2} b_{j}^{\mathrm{NS}}\right), \quad i=1, \cdots, g_{\Sigma} .
$$

It follows from this equation that the 't Hooft parameters are flat coordinates on the moduli space. The frame defined by these coordinates will be called the maximal conifold frame (MCF). The submanifold in moduli space defined by

$$
\lambda_{i}=0, \quad i=1, \cdots, g_{\Sigma},
$$

has dimension $r_{\Sigma}$ (the number of mass parameters of the toric CY), and we will call it the maximal conifold locus (MCL). It is a submanifold of the conifold locus of the CY X. By 
evaluating the integral (2.58) in the saddle-point approximation, we find that the fermionic spectral traces have the following asymptotic expansion in the 't Hooft limit:

$$
\log Z(\boldsymbol{N} ; \hbar) \sim \sum_{g \geq 0} \mathcal{F}_{g}(\boldsymbol{\lambda}) \hbar^{2-2 g}
$$

The leading function in this expansion is given by a Legendre transform,

$$
\mathcal{F}_{0}(\boldsymbol{\lambda})=\mathrm{J}_{0}^{X}(\boldsymbol{\zeta}, \boldsymbol{\xi})-\boldsymbol{\lambda} \cdot \boldsymbol{\zeta}
$$

If we choose the Batyrev coordinates in such a way that the first $g_{\Sigma}$ correspond to true moduli, and the remaining $r_{\Sigma}$ correspond to mass parameters, we find

$$
\frac{\partial \mathcal{F}_{0}}{\partial \lambda_{i}}=-\zeta_{i}=-\sum_{j=1}^{g_{\Sigma}} \frac{C_{i j}^{-1}}{2 \pi}\left(T_{j}-\sum_{k=1}^{r_{\Sigma}} \alpha_{j k} \log \xi_{k}\right), \quad i=1, \cdots, g_{\Sigma},
$$

where $C^{-1}$ denotes the inverse of the truncated matrix (2.36). In view of the results of [55], the higher order corrections $\mathcal{F}_{g}(\boldsymbol{\lambda})$ can be computed in a very simple way: the integral (2.58) implements a symplectic transformation from the LRF to the MCF. The functions $\mathcal{F}_{g}(\boldsymbol{\lambda})$ are precisely the genus $g$ free energies of the topological string in the MCF.

\subsection{Perturbed $\mathrm{O}_{m, n}$ operators}

In order to test the conjectures of $[1,27]$, it is very useful to have explicit results on the spectral theory side. In particular, since we have a precise conjecture for the values of the fermionic spectral traces of the relevant operators in terms of enumerative invariants, we would like to have independent, analytic computations of these traces.

In many cases, the operators which appear in the quantization of mirror curves are perturbations of three-term operators of the form

$$
\mathrm{O}_{m, n}=\mathrm{e}^{\mathrm{x}}+\mathrm{e}^{\mathrm{y}}+\mathrm{e}^{-m \mathrm{x}-n \mathrm{y}}, \quad m, n \in \mathbb{Z}_{>0} .
$$

These operators were introduced and studied in [18]. It turns out that the integral kernel of their inverses $\rho_{m, n}=\mathrm{O}_{m, n}^{-1}$ can be explicitly computed in terms of Faddeev's quantum dilogarithm. This makes it possible to calculate the standard traces

$$
\operatorname{Tr} \rho_{m, n}^{\ell}, \quad \ell=1,2, \cdots,
$$

in terms of integrals over the real line. These integrals can be computed by using the techniques of [56], or by using the recursive methods developed in [12, 22, 57, 58]. Once the "bosonic" spectral traces (2.73) have been computed, the fermionic spectral traces follow by simple combinatorics. Mixed traces, as those appearing in (2.30), (2.31), can be also obtained in terms of integrals.

In this section, we will study a four-term operator which is a perturbation of (2.72). This operator reads,

$$
\mathrm{O}_{m, n, \xi}=\mathrm{e}^{\mathrm{x}}+\mathrm{e}^{\mathrm{y}}+\mathrm{e}^{-m \mathrm{x}-n \mathrm{y}}+\xi \mathrm{e}^{-(1+m) \mathrm{x}-(n-1) \mathrm{y}}, \quad m, n \in \mathbb{Z}_{>0} .
$$


Let us introduce the parameter $\zeta$ by $^{5}$

$$
\xi=\mathrm{e}^{2 \pi \mathrm{b} \zeta}
$$

We will now obtain an explicit expression for the integral kernel of

$$
\rho_{m, n, \xi}=\mathrm{O}_{m, n, \xi}^{-1},
$$

based on similar derivations in [18] and [20]. First, as in [18], we introduce the Heisenberg operators $\mathrm{q}$ and $\mathrm{p}$ satisfying the normalized commutation relations

$$
[\mathrm{p}, \mathrm{q}]=\frac{1}{2 \pi \mathrm{i}}
$$

They are related to the operators $\mathrm{x}, \mathrm{y}$ appearing in (2.74) by

$$
\mathrm{x} \equiv 2 \pi \mathrm{b} \frac{(n+1) \mathrm{p}+n \mathrm{q}}{m+n+1}, \quad \mathrm{y} \equiv-2 \pi \mathrm{b} \frac{m \mathrm{p}+(m+1) \mathrm{q}}{m+n+1},
$$

so that

$$
\hbar=\frac{2 \pi \mathrm{b}^{2}}{m+n+1}
$$

We then have,

$$
\begin{aligned}
\mathrm{e}^{-\mathrm{y} / 2} \mathrm{O}_{m, n, \xi} \mathrm{e}^{-\mathrm{y} / 2} & =\mathrm{e}^{\mathrm{x}-\mathrm{y}}+1+\mathrm{e}^{-m \mathrm{x}-(n+1) \mathrm{y}}+\xi r \mathrm{e}^{-(1+m) \mathrm{x}-n \mathrm{y}} \\
& =\mathrm{e}^{2 \pi \mathrm{b}(\mathrm{p}+\mathrm{q})}+1+\mathrm{e}^{2 \pi \mathrm{bq}}+\xi \mathrm{e}^{-2 \pi \mathrm{bp}} \\
& =\mathrm{e}^{-\pi \mathrm{bp}}\left(\mathrm{e}^{2 \pi \mathrm{b}(2 \mathrm{p}+\mathrm{q})}+\mathrm{e}^{2 \pi \mathrm{b} p}+\mathrm{e}^{2 \pi \mathrm{bq}}+\xi\right) \mathrm{e}^{-\pi \mathrm{bp}} .
\end{aligned}
$$

We now use Faddeev's quantum dilogarithm $\Phi_{\mathrm{b}}(x)$ [59-61] (our conventions for this function are as in [18]). It satisfies the following identity (a similar identity was already used in [18]):

$$
\Phi_{b}(p) \Phi_{b}^{*}(q) e^{2 \pi b p} \Phi_{b}(q) \Phi_{b}^{*}(p)=e^{2 \pi b(2 p+q)}+e^{2 \pi b p}+e^{2 \pi b(p+q)} .
$$

Its behaviour under complex conjugation is given by,

$$
\Phi_{\mathrm{b}}^{*}(z)=\frac{1}{\Phi_{\mathrm{b}}\left(z^{*}\right)} .
$$

Let us denote

$$
\widehat{\mathrm{O}}_{m, n, \xi}=\mathrm{e}^{\pi \mathrm{bp}-\mathrm{y} / 2} \mathrm{O}_{m, n, \xi} \mathrm{e}^{\pi \mathrm{bp}-\mathrm{y} / 2} .
$$

We now recall that Faddeev's quantum dilogarithm satisfies the following difference equation,

$$
\frac{\Phi_{\mathrm{b}}\left(x+c_{\mathrm{b}}+\mathrm{ib}\right)}{\Phi_{\mathrm{b}}\left(x+c_{\mathrm{b}}\right)}=\frac{1}{1-q \mathrm{e}^{2 \pi \mathrm{b} x}},
$$

where

$$
q=\mathrm{e}^{2 \pi \mathrm{i}^{2}}, \quad c_{\mathrm{b}}=\mathrm{i} \frac{\mathrm{b}+\mathrm{b}^{-1}}{2} .
$$

\footnotetext{
${ }^{5}$ Do not confuse the $\zeta$ introduced here with the 't Hooft variables defined in (2.60).
} 
By using this equation, we obtain

$$
\begin{aligned}
\Phi_{\mathrm{b}}(\mathrm{q}) \Phi_{\mathrm{b}}^{*}(\mathrm{p}) \widehat{\mathrm{O}}_{m, n, \xi} \Phi_{\mathrm{b}}(\mathrm{p}) \Phi_{\mathrm{b}}^{*}(\mathrm{q}) & =\xi+\mathrm{e}^{2 \pi \mathrm{bp}}=\xi\left(1+\mathrm{e}^{2 \pi \mathrm{b}(\mathrm{p}-\zeta)}\right) \\
& =\xi \frac{\Phi_{\mathrm{b}}(\mathrm{p}-\zeta-\mathrm{ib} / 2)}{\Phi_{\mathrm{b}}(\mathrm{p}-\zeta+\mathrm{ib} / 2)}
\end{aligned}
$$

By taking the inverse, we find,

$$
\Phi_{\mathrm{b}}(\mathrm{q}) \Phi_{\mathrm{b}}^{*}(\mathrm{p}) \mathrm{e}^{-\pi \mathrm{b} p+\mathrm{y} / 2} \xi \mathrm{O}_{m, n, \xi}^{-1} \mathrm{e}^{-\pi \mathrm{bp}+\mathrm{y} / 2} \Phi_{\mathrm{b}}(\mathrm{p}) \Phi_{\mathrm{b}}^{*}(\mathrm{q})=\frac{\Phi_{\mathrm{b}}(\mathrm{p}-\zeta+\mathrm{ib} / 2)}{\Phi_{\mathrm{b}}(\mathrm{p}-\zeta-\mathrm{ib} / 2)},
$$

and we can write,

$$
\xi \mathrm{O}_{m, n, \xi}^{-1}=\mathrm{e}^{\pi \mathrm{b} p-y / 2} \Phi_{\mathrm{b}}(\mathrm{p}) \Phi_{\mathrm{b}}^{*}(\mathrm{q}) \frac{1}{\Phi_{\mathrm{b}}(\mathrm{p}-\zeta-\mathrm{ib} / 2)} \Phi_{\mathrm{b}}(\mathrm{p}-\zeta+\mathrm{ib} / 2) \Phi_{\mathrm{b}}(\mathrm{q}) \Phi_{\mathrm{b}}^{*}(\mathrm{p}) \mathrm{e}^{\pi \mathrm{b} p-y / 2} .
$$

We now use the quantum pentagon identity [60]

$$
\Phi_{\mathrm{b}}\left(\mathrm{p}-p_{0}\right) \Phi_{\mathrm{b}}(\mathrm{q})=\Phi_{\mathrm{b}}(\mathrm{q}) \Phi_{\mathrm{b}}\left(\mathrm{p}-p_{0}+\mathrm{q}\right) \Phi_{\mathrm{b}}\left(\mathrm{p}-p_{0}\right),
$$

where $p_{0}=\zeta-\mathrm{ib} / 2$, to obtain

$$
\xi \mathrm{O}_{m, n, \xi}^{-1}=\mathrm{e}^{\pi \mathrm{bp}-\mathrm{y} / 2} \frac{\Phi_{\mathrm{b}}(\mathrm{p})}{\Phi_{\mathrm{b}}\left(\mathrm{p}-p_{0}^{*}\right)} \frac{1}{1+\mathrm{e}^{2 \pi \mathrm{b}(\mathrm{p}+\mathrm{q}-\zeta)}} \frac{\Phi_{\mathrm{b}}\left(\mathrm{p}-p_{0}\right)}{\Phi_{\mathrm{b}}(\mathrm{p})} \mathrm{e}^{\pi \mathrm{bp}-\mathrm{y} / 2}
$$

Let us now introduce the parameters [18]

$$
a=\frac{\mathrm{b} m}{2(m+n+1)}, \quad c=\frac{\mathrm{b}}{2(m+n+1)}, \quad h=a+c-n c,
$$

as well as the function

$$
\Psi_{a, c}(x) \equiv \frac{\mathrm{e}^{2 \pi a x}}{\Phi_{\mathrm{b}}(x-\mathrm{i}(a+c))} .
$$

We will also relabel

$$
p+q-\zeta \rightarrow q
$$

Then, we have

$$
\rho_{m, n, \xi}=\mathrm{O}_{m, n, \xi}^{-1}=\Psi_{a, c}^{*}(\mathrm{p}) \Psi_{n c, 0}(\mathrm{p}-\zeta)\left|\Psi_{a+c, c n}(\mathrm{q})\right|^{2} \Psi_{a, c}(\mathrm{p}) \Psi_{n c, 0}^{*}(\mathrm{p}-\zeta),
$$

where we used again the property (2.84). In particular, its kernel can be written, in the momentum representation

$$
\mathrm{p} \mid x)=x \mid x)
$$

as

$$
\left(x\left|\rho_{m, n, \xi}\right| y\right)=\rho_{m, n, \xi}(x, y)=\frac{\overline{f(x)} f(y)}{2 \mathrm{~b} \cosh \left(\pi \frac{x-y+\mathrm{i} h}{\mathrm{~b}}\right)},
$$

where

$$
f(x)=\Psi_{a, c}(x) \Psi_{n c, 0}^{*}(x-\zeta)=\frac{\Phi_{\mathrm{b}}(x-\zeta+\mathrm{i} n c)}{\Phi_{\mathrm{b}}(x-\mathrm{i}(a+c))} \mathrm{e}^{2 \pi(a+n c) x} \mathrm{e}^{-2 \pi c n \zeta}
$$


It can be easily checked, by using the properties of Faddeev's quantum dilogarithm, that as $\xi \rightarrow 0$ (which corresponds to $\zeta \rightarrow-\infty$ ), we recover the kernel of $\mathrm{O}_{m, n}$ determined in [18].

Using the results (2.96), (2.97), it is possible to compute explicitly the spectral traces of $\rho_{m, n, \xi}$. Let us consider an example which will be relevant for the conjectural relation with the topological string: we consider $m=n=1$, so that we consider a perturbation of the operator associated to local $\mathbb{P}^{2}[1]$. Let us focus on the maximally supersymmetric case $\hbar=2 \pi$, which corresponds to $b=\sqrt{3}$. The diagonal integral kernel is given by

$$
\begin{aligned}
\rho_{1,1, \xi}(x, x) & =\frac{1}{2 \mathrm{~b} \cos (\pi / 6)} \mathrm{e}^{4 \pi x / \mathrm{b}-2 \pi \zeta / \mathrm{b}} \frac{\Phi_{\mathrm{b}}(x+\mathrm{ib} / 3)}{\Phi_{\mathrm{b}}(x-\mathrm{ib} / 3)} \frac{\Phi_{\mathrm{b}}(x-\zeta+\mathrm{ib} / 6)}{\Phi_{\mathrm{b}}(x-\zeta-\mathrm{ib} / 6)} \\
& =\frac{1}{2 \mathrm{~b} \cos (\pi / 6)} \frac{\mathrm{e}^{4 \pi x / \mathrm{b}-2 \pi \zeta / \mathrm{b}}}{\left(1+\mathrm{e}^{2 \pi x / \mathrm{b}}+\mathrm{e}^{4 \pi x / \mathrm{b}}\right)\left(1+\mathrm{e}^{2 \pi(x-\zeta) / \mathrm{b}}\right)} .
\end{aligned}
$$

This can be integrated, to obtain

$$
\operatorname{Tr} \rho_{1,1, \xi}=\frac{\pi\left(2-\xi^{1 / 3}\right)+\sqrt{3} \xi^{1 / 3} \log (\xi)}{18 \pi\left(\xi^{2 / 3}-\xi^{1 / 3}+1\right)} .
$$

Similarly, one finds ${ }^{6}$

$$
\begin{aligned}
\operatorname{Tr} \rho_{1,1, \xi}^{2}= & -\frac{6 \sqrt{3}(1+\xi)+\pi\left(-4+4 \xi^{1 / 3}-13 \xi^{2 / 3}+6 \xi\right)}{108 \pi\left(1+\xi^{1 / 3}\right)\left(1-\xi^{1 / 3}+\xi^{2 / 3}\right)^{2}} \\
& +\frac{\xi^{2 / 3} \log \xi}{18 \sqrt{3} \pi\left(1-\xi^{1 / 3}+\xi^{2 / 3}\right)^{2}}+\frac{\xi^{2 / 3}(\log \xi)^{2}}{36 \pi^{2}\left(1+\xi^{1 / 3}\right)\left(1-\xi^{1 / 3}+\xi^{2 / 3}\right)^{2}}
\end{aligned}
$$

These traces are functions of $\xi$ with a branch cut at $\xi=0$. Their limit when $\xi \rightarrow 0$ exists and gives back the traces for the operator $\rho_{1,1} \equiv \rho_{1,1,0}$ calculated in for example [18]. The theory for $\xi=1$ is particularly simple and we find

$$
\operatorname{Tr} \rho_{1,1, \xi=1}=\frac{1}{18}, \quad \operatorname{Tr} \rho_{1,1, \xi=1}^{2}=\frac{7}{216}-\frac{1}{6 \sqrt{3} \pi} .
$$

\section{The $Y^{3,0}$ geometry}

In this section, we test the conjectural correspondence between spectral theory and topological strings in a genus two example with one mass parameter, namely the $Y^{3,0}$ geometry.

\subsection{Mirror curve and operator content}

The generic $Y^{N, q}$ geometry ${ }^{7}$ has been studied in some detail in [62]. The mirror curve is given by

$$
a_{1} \mathrm{e}^{p}+a_{2} \mathrm{e}^{-p+(N-q) x}+\sum_{i=0}^{N} b_{i} \mathrm{e}^{i x}=0,
$$

\footnotetext{
${ }^{6}$ We would like to thank Szabolcs Zakany for adapting the techniques of [22] to check this result.

${ }^{7}$ To be precise, we are talking about the resolution of the cone over the $Y^{N, q}$ singularity.
} 
where $p, x$ are variables. Here we are interested in the case $q=0$. In particular, when $N=3, q=0$, the mirror curve has the form

$$
a_{1} \mathrm{e}^{p}+a_{2} \mathrm{e}^{-p+3 x}+b_{3} \mathrm{e}^{3 x}+b_{2} \mathrm{e}^{2 x}+b_{1} \mathrm{e}^{x}+b_{0}=0 .
$$

The corresponding charge vectors are

$$
Q^{1}=(0,0,1,-2,1,0), \quad Q^{2}=(0,0,0,1,-2,1), \quad Q^{3}=(1,1,-1,0,0,-1),
$$

and the Batyrev coordinates are

$$
z_{1}=\frac{b_{3} b_{1}}{b_{2}^{2}}, \quad z_{2}=\frac{b_{0} b_{2}}{b_{1}^{2}}, \quad z_{3}=\frac{a_{1} a_{2}}{b_{3} b_{0}} .
$$

The canonical forms of the mirror curve (3.2) are

$$
\begin{aligned}
& b_{3} \mathrm{e}^{x}+\mathrm{e}^{y}+\mathrm{e}^{-x-y}+b_{0} \mathrm{e}^{-2 x}+b_{1} \mathrm{e}^{-x}+b_{2}=0, \\
& b_{0} \mathrm{e}^{u}+\mathrm{e}^{y}+\mathrm{e}^{-u-y}+b_{3} \mathrm{e}^{-2 u}+b_{2} \mathrm{e}^{-u}+b_{1}=0,
\end{aligned}
$$

where we set $a_{1}=a_{2}=1$. To obtain the first curve, start from (3.2) and set

$$
p=y+2 x .
$$

After multiplying the resulting equation by $\mathrm{e}^{-2 x}$, we obtain precisely the first curve in (3.5). To obtain the second curve, we multiply (3.2) by $\mathrm{e}^{-x}$ and we perform the symplectic transformation from $(p, x)$ to $(y, u)$

$$
y=2 x-p, \quad u=-x .
$$

Note that both curves are identical after exchanging $b_{1} \leftrightarrow b_{2}$ and $b_{0} \leftrightarrow b_{3}$. We regard $b_{1}, b_{2}$ as moduli of the curve, and $b_{3}, b_{0}$ as parameters. We can further set one of $b_{0}, b_{3}$ to one by using the remaining $\mathbb{C}^{*}$ rescaling freedom, but we refrain from doing it to make the symmetry between the two canonical forms in (3.5) apparent.

The operators $\mathrm{O}_{i}^{(0)}, i=1,2$, obtained by quantization of the canonical forms are

$$
\begin{aligned}
& \mathrm{O}_{1}^{(0)}=b_{3} \mathrm{e}^{\mathrm{x}}+\mathrm{e}^{\mathrm{y}}+\mathrm{e}^{-\mathrm{x}-\mathrm{y}}+b_{0} \mathrm{e}^{-2 \mathrm{x}}, \\
& \mathrm{O}_{2}^{(0)}=b_{0} \mathrm{e}^{\mathrm{u}}+\mathrm{e}^{\mathrm{y}}+\mathrm{e}^{-\mathrm{u}-\mathrm{y}}+b_{3} \mathrm{e}^{-2 \mathrm{u}} .
\end{aligned}
$$

Both of them can be regarded as perturbations of the operator $\mathrm{O}_{1,1}$ introduced in (2.72), up to an overall normalization. In the first case, we change the normalization of $x, y$ in such a way that

$$
\mathrm{e}^{\mathrm{x}} \rightarrow b_{3}^{-2 / 3} \mathrm{e}^{\mathrm{x}}, \quad \mathrm{e}^{\mathrm{y}} \rightarrow b_{3}^{1 / 3} \mathrm{e}^{\mathrm{y}},
$$

and then we find

$$
b_{3} \mathrm{e}^{\mathrm{x}}+\mathrm{e}^{\mathrm{y}}+\mathrm{e}^{-\mathrm{x}-\mathrm{y}}+b_{0} \mathrm{e}^{-2 \mathrm{x}}+b_{1} \mathrm{e}^{-\mathrm{x}} \rightarrow b_{3}^{1 / 3}\left(\mathrm{e}^{\mathrm{x}}+\mathrm{e}^{\mathrm{y}}+\mathrm{e}^{-\mathrm{x}-\mathrm{y}}+b_{3} b_{0} \mathrm{e}^{-2 \mathrm{x}}+b_{1} b_{3}^{1 / 3} \mathrm{e}^{-\mathrm{x}}\right),
$$

Note that, when $b_{1}=0$, the operator inside the parentheses is precisely the operator $O_{1,1, \xi}$ introduced in (2.74), where

$$
\xi=b_{3} b_{0}=\frac{1}{z_{3}}
$$


In the second operator the normalization is fixed by requiring

$$
\mathrm{e}^{\mathrm{u}} \rightarrow b_{0}^{-2 / 3} \mathrm{e}^{\mathrm{u}}, \quad \mathrm{e}^{\mathrm{y}} \rightarrow b_{0}^{1 / 3} \mathrm{e}^{\mathrm{y}},
$$

and then we find

$$
b_{0} \mathrm{e}^{\mathrm{u}}+\mathrm{e}^{\mathrm{y}}+\mathrm{e}^{-\mathrm{u}-\mathrm{y}}+b_{3} \mathrm{e}^{-2 \mathrm{u}}+b_{2} \mathrm{e}^{-\mathrm{u}} \rightarrow b_{0}^{1 / 3}\left(\mathrm{e}^{\mathrm{u}}+\mathrm{e}^{\mathrm{y}}+\mathrm{e}^{-\mathrm{u}-\mathrm{y}}+b_{3} b_{0} \mathrm{e}^{-2 \mathrm{u}}+b_{2} b_{0}^{1 / 3} \mathrm{e}^{-\mathrm{u}}\right),
$$

Note in particular that

$$
\begin{aligned}
& \operatorname{Tr}\left(\rho_{1}^{(0)}\right)^{N}=b_{3}^{-N / 3} \operatorname{Tr} \rho_{1,1, \xi}^{N}, \\
& \operatorname{Tr}\left(\rho_{2}^{(0)}\right)^{N}=b_{0}^{-N / 3} \operatorname{Tr} \rho_{1,1, \xi}^{N} .
\end{aligned}
$$

In the following, we will set

$$
\kappa_{1}=b_{2}, \quad \kappa_{2}=b_{1} .
$$

Note that the truncated matrix appearing in (2.36) is in this case the Cartan matrix of $\mathrm{SU}(3)$,

$$
C=\left(\begin{array}{cc}
2 & -1 \\
-1 & 2
\end{array}\right)
$$

with inverse

$$
C^{-1}=\frac{1}{3}\left(\begin{array}{ll}
2 & 1 \\
1 & 2
\end{array}\right) .
$$

\section{2 't Hooft expansion of the fermionic traces}

One of the main points of $[1,27]$, as we reviewed in section 2.2 , is that the topological string amplitudes in the MCF can be obtained from the 't Hooft limit (2.59) of the fermionic spectral traces (2.24). Therefore, we have the asymptotic expansion given in (2.69). When the genus of the mirror curve is two, we can write

$$
\log Z\left(N_{1}, N_{2} ; \hbar\right)=\mathcal{F}\left(\lambda_{1}, \lambda_{2} ; \hbar\right)=\sum_{g=0}^{\infty} \hbar^{2-2 g} \mathcal{F}_{g}\left(\lambda_{1}, \lambda_{2}\right)
$$

where $\mathcal{F}_{g}\left(\lambda_{1}, \lambda_{2}\right)$ is the topological string free energy at genus $g$ in the MCF, and the 't Hooft parameters $\lambda_{i}$ are identified with suitable flat coordinates in the MCF as specified in (2.67). The genus $g$ topological string free energy can be in turn expanded around the MCL $\lambda_{1}=\lambda_{2}=0$, and one finds

$$
\mathcal{F}_{g}\left(\lambda_{1}, \lambda_{2}\right)=\mathcal{F}^{\log }\left(\lambda_{1}, \lambda_{2}\right) \delta_{g, 0}+\sum_{\substack{i, j=0 \\(i, j) \neq(0,0)}}^{\infty} \mathcal{F}_{g ; i, j} \lambda_{1}^{i} \lambda_{2}^{j}
$$

where $\mathcal{F}^{\log }\left(\lambda_{1}, \lambda_{2}\right)$ contains $\log \lambda_{1}, \log \lambda_{2}$, and the remaining part is a power series in $\lambda_{1}, \lambda_{2}$. The numbers $\mathcal{F}_{g ; i, j}$ can be regarded as "conifold" Gromov-Witten invariants. In order to extract these invariants from the fermionic traces, there are various techniques that we can use. First of all, note that if $N_{1}=0$ or $N_{2}=0$, the fermionic traces can be computed 
from a matrix model with a single set of eigenvalues, as in $[19,20]$. The matrix integral is given by

$$
Z_{m, n, \xi}(N, \hbar)=\frac{1}{N !} \int_{\mathbb{R}^{N}} \frac{\mathrm{d}^{N} u}{(2 \pi)^{N}} \prod_{i=1}^{N} \mathrm{e}^{-\frac{1}{\mathrm{~g}} V_{m, n, \xi}\left(u_{i}, \mathrm{~g}\right)} \frac{\prod_{i<j} 4 \sinh \left(\frac{u_{i}-u_{j}}{2}\right)^{2}}{\prod_{i, j} 2 \cosh \left(\frac{u_{i}-u_{j}}{2}+\mathrm{i} \pi C_{m, n}\right)},
$$

where

$$
\mathrm{g}=\frac{m+n+1}{2 \pi \mathrm{b}^{2}}, \quad C_{m, n}=\frac{m-n+1}{2(m+n+1)} .
$$

The potential $V_{m, n, \xi}(u, \mathrm{~g})$ is given by

$$
V_{m, n, \xi}(u, \mathrm{~g})=-\mathrm{g}\left(\frac{(m+n) \mathrm{b}^{2}}{m+n+1} u-\frac{2 \pi \mathrm{b} n \zeta}{m+n+1}\right)-2 \mathrm{~g} \log \left|\frac{\Phi_{\mathrm{b}}(p+\mathrm{i}(a+c))}{\Phi_{\mathrm{b}}(p-\zeta-\mathrm{i} c n)}\right|,
$$

where $u$ is related to $p$ by

$$
u=\frac{2 \pi}{\mathrm{b}} p
$$

while $\xi$ is related to $\zeta$ by (2.75). The values of $a, c$ are given in (2.91). Then, due to the correction in (3.14), we find the following formulae for the fermionic traces:

$$
\begin{aligned}
& \log Z(N, 0 ; \hbar)=\log Z_{1,1, \xi}(N, \hbar)-\frac{\hbar^{2} \lambda}{2 \pi} \frac{1}{3} \log \widehat{b}_{3}, \\
& \log Z(0, N ; \hbar)=\log Z_{1,1, \xi}(N, \hbar)-\frac{\hbar^{2} \lambda}{2 \pi} \frac{1}{3} \log \widehat{b}_{0},
\end{aligned}
$$

where the hatted parameters are defined as in (2.61), i.e.

$$
\widehat{b}_{3}=b_{3}^{2 \pi / \hbar}, \quad \widehat{b}_{0}=b_{0}^{2 \pi / \hbar} .
$$

In practice, the expansion (3.19) can be computed as follows. Replacing $\lambda_{i}$ by $N_{i} / \hbar$, one gets

$$
\log Z\left(N_{1}, N_{2} ; \hbar\right)=\mathcal{F}^{\log }\left(N_{1} / \hbar, N_{2} / \hbar\right) \hbar^{-2}+\sum_{n \geqslant-1} \hbar^{-n} \sum_{\substack{i, j \geqslant 0,(i, j) \neq(0,0) \\ i+j \equiv n \bmod 2}}^{i+j \leqslant n+2} \mathcal{F}_{\frac{n-i-j}{2}+1 ; i, j} N_{1}^{i} N_{2}^{j}
$$

Note that since $\mathcal{F}^{\log }\left(\lambda_{1}, \lambda_{2}\right) \sim \log \left(\lambda_{i}\right) \lambda_{i}^{2}$, the first term $\mathcal{F}^{\log }\left(N_{1} / \hbar, N_{2} / \hbar\right) \hbar^{-2}$ is of the order $\mathcal{O}\left(\hbar^{0}\right)$. We find out that in the power series part of $\log Z\left(N_{1}, N_{2} ; \hbar\right)$, only a finite number of MCF Gromov-Witten invariants contributes at each order $\hbar^{-n}$. Therefore a simple linear algebra calculation allows us to extract the numbers $\mathcal{F}_{g ; i, j}$ from the large $\hbar$ expansion of $\log Z\left(N_{1}, N_{2} ; \hbar\right)$, evaluated with different values of $N_{1}, N_{2}$. For instance,

$$
\begin{aligned}
\mathcal{F}_{0 ; 3,0} & =\left.\frac{1}{6}(-2 \log Z(1,0)+\log Z(2,0))\right|_{\hbar^{-1}}, \\
\mathcal{F}_{1 ; 1,0} & =\left.\frac{1}{6}(8 \log Z(1,0)-\log Z(2,0))\right|_{\hbar^{-1}}, \\
\mathcal{F}_{0 ; 2,1} & =\left.\frac{1}{2}(\log Z(0,1)+2 \log Z(1,0)-2 \log Z(1,1)-\log Z(2,0)+\log Z(2,1))\right|_{\hbar^{-1}} .
\end{aligned}
$$


In the above expressions, we suppress "; $\hbar$ " in $Z\left(N_{1}, N_{2} ; \hbar\right)$ to simplify the notation. We use $\left.\right|_{\hbar^{-n}}$ to denote the contribution of the power series at the order $\hbar^{-n}$.

To evaluate $Z\left(N_{1}, N_{2} ; \hbar\right)$ directly, we should choose a canonical form of the quantum mirror curve, read off the operators $\mathrm{A}_{j 1}, \mathrm{~A}_{j 2}$, and compute the fermionic traces in terms of the spectral traces of $\mathrm{A}_{j 1}, \mathrm{~A}_{j 2}$ by (2.29). We will choose $j=1$, simplify notation by setting $\mathrm{A}_{1 i}=\mathrm{A}_{i}, i=1,2$, and put $b_{3}=1$ for simplicity. The mass parameter $\xi$ is then $b_{0}$. It is easy to see that

$$
\mathrm{A}_{1}=\rho_{1,1, \xi}, \quad \mathrm{A}_{2}=\rho_{1,1, \xi} \mathrm{e}^{-\mathrm{x}} .
$$

The operator $\rho_{1,1, \xi}$ is an operator of the type $\rho_{m, n, \xi}$ studied in detail in section 2.3. Its kernel in the $p$-space (recall the relation between the canonical variables $(x, y)$ and the canonical variables $(p, q)$ in (2.78) as well as the relabeling (2.93)) is given in (2.96), (2.97) with $m=n=1$. Working carefully in the space of the variable $p$, we find the kernel of the second operator

$$
A_{2}(x, y)=\mathrm{e}^{-\frac{\pi \mathrm{i}}{9} \mathrm{~b}^{2}} \mathrm{e}^{-\frac{2 \pi \mathrm{b} y}{3}} \rho_{1,1, \xi}\left(x, y+\frac{\mathrm{ib}}{3}\right) .
$$

Recall that $\hbar$ is proportional to $b$ by (2.79). To perform the large $\hbar$ expansion of the traces, we need the large $b$ expansion of the Faddeev's quantum dilogarithm

$$
\begin{aligned}
\log \Phi_{\mathrm{b}}(x+\delta \mathrm{b}) & =\frac{\mathrm{b}^{2}}{2 \pi \mathrm{i}} \sum_{k=0}^{\infty} \mathrm{b}^{-k} \sum_{j=0}^{\lfloor k / 4\rfloor} \operatorname{Li}_{2+2 j-k}\left(-\mathrm{e}^{2 \pi \delta}\right) \frac{(2 \pi)^{k-2 j}(-1)^{j} B_{2 j}(1 / 2)}{(k-4 j) !(2 j) !} x^{k-4 j} \\
& =-\mathrm{ib}^{2} \frac{\operatorname{Li}_{2}\left(-\mathrm{e}^{2 \pi \delta}\right)}{2 \pi}+\mathrm{ib} \log \left(1+\mathrm{e}^{2 \pi \delta}\right) x+\mathcal{O}\left(\mathrm{b}^{-1}\right),
\end{aligned}
$$

where $B_{2 n}(x)$ are Bernoulli polynomials, and $\delta$ an arbitrary constant. Then, for instance, $\operatorname{Tr} A_{1}$ has the following form

$$
\operatorname{Tr} \mathrm{A}_{1}=\int_{-\infty}^{\infty} \mathrm{d} x \mathrm{~A}_{1}(x, x)=\int_{-\infty}^{\infty} \mathrm{d} x \mathrm{e}^{\alpha \mathrm{b}^{2}+\mathrm{i} \beta \mathrm{b} x-\gamma x^{2}} \sum_{k=0}^{\infty} \mathrm{b}^{-k} p_{k}(x),
$$

where $p_{k}(x)$ is a polynomial in $x$. To facilitate the calculation of the integral, one can eliminate the linear term $\mathrm{i} \beta \mathrm{b} x$ in the exponential by giving an appropriate shift $\delta \mathrm{b}$ to $x$ before expanding the quantum dilogarithms, such that at each order of $\mathrm{b}^{-k}$, one has a Gaussian integral or a derivative thereof. The same trick can be applied when the other spectral traces are evaluated.

To simplify the calculation, we take $\xi=1$. Then we obtain the traces of $A_{1}$, for instance

$$
\begin{aligned}
& \operatorname{Tr} \mathrm{A}_{1}=o\left(\mathrm{~b}^{0}\right)+\frac{4 \pi^{2}}{75 \sqrt{3} \mathrm{~b}^{4}}-\frac{116 \pi^{3}}{3375 \sqrt{5} \mathrm{~b}^{6}}+\frac{452 \pi^{4}}{28125 \sqrt{3} \mathrm{~b}^{8}}+\mathcal{O}\left(\mathrm{b}^{-9}\right), \\
& \operatorname{Tr} \mathrm{A}_{1}^{2}=o\left(\mathrm{~b}^{0}\right)-\frac{8 \pi}{9 \sqrt{15} \mathrm{~b}^{2}}+\frac{152 \pi^{2}}{675 \mathrm{~b}^{4}}-\frac{12616 \pi^{3}}{10125 \sqrt{15} \mathrm{~b}^{6}}+\frac{1575688 \pi^{4}}{2278125 \mathrm{~b}^{8}}+\mathcal{O}\left(\mathrm{b}^{-9}\right),
\end{aligned}
$$

as well as the mixed traces of $A_{1}$ and $A_{2}$

$$
\begin{aligned}
& \operatorname{Tr} \mathrm{A}_{1} \mathrm{~A}_{2}=o\left(\mathrm{~b}^{0}\right)-\frac{2 \pi}{5 \sqrt{15} \mathrm{~b}^{2}}+\frac{26 \pi^{2}}{375 \mathrm{~b}^{4}}-\frac{1468 \pi^{3}}{5625 \sqrt{15} \mathrm{~b}^{6}}+\frac{47242 \pi^{4}}{421875 \mathrm{~b}^{8}}+\mathcal{O}\left(\mathrm{b}^{-9}\right) \\
& \operatorname{Tr} \mathrm{A}_{1}^{2} \mathrm{~A}_{2}=o\left(\mathrm{~b}^{0}\right)-\frac{58 \pi}{225 \sqrt{5} \mathrm{~b}^{2}}+\frac{1138 \pi^{2}}{5625 \sqrt{3} \mathrm{~b}^{4}}-\frac{17968 \pi^{3}}{50625 \sqrt{5} \mathrm{~b}^{6}}+\frac{10393358 \pi^{4}}{18984375 \sqrt{3} \mathrm{~b}^{8}}+\mathcal{O}\left(\mathrm{b}^{-9}\right) .
\end{aligned}
$$


Here $o\left(\mathrm{~b}^{0}\right)$ means only terms of the order $\mathrm{b}^{0}$. In addition, (3.14) implies that the traces of $\mathrm{A}_{2}$ are straightforwardly related to those of $\mathrm{A}_{1}$. In fact when $\xi=1$, one can show that

$$
\operatorname{Tr} \mathrm{A}_{1}^{r} \mathrm{~A}_{2}^{s}=\operatorname{Tr}_{1}^{s} \mathrm{~A}_{2}^{r}
$$

Once we have the large $b$ expansion of the traces of $A_{1}, A_{2}$, we can easily extract the MCF Gromov-Witten invariants. When $\xi=1$, we get for instance

$$
\begin{array}{lll}
\mathcal{F}_{0 ; 3,0}=-\frac{16 \pi^{2}}{45 \sqrt{15}}, & \mathcal{F}_{0 ; 4,0}=\frac{866 \pi^{4}}{30375}, & \mathcal{F}_{0 ; 5,0}=-\frac{102848 \pi^{6}}{1366875 \sqrt{15}}, \\
\mathcal{F}_{1 ; 1,0}=\frac{16 \pi^{2}}{45 \sqrt{15}}, & \mathcal{F}_{1 ; 2,0}=-\frac{146 \pi^{4}}{30375}, & \mathcal{F}_{1 ; 3,0}=-\frac{9472 \pi^{6}}{1366875 \sqrt{15}}, \\
\mathcal{F}_{0 ; 2,1}=\mathcal{F}_{0 ; 1,2}=\frac{\pi^{2}}{\sqrt{15}} . &
\end{array}
$$

We will check these numbers against the computation in the topological string theory.

Another set of quantities that can be easily computed from operator theory are the derivatives of the genus zero free energy at the MCL. To do this computation, we first note that, as shown in [19], the linear term in $\lambda$ of the planar free energy of the matrix integral (3.20) is given by the "classical" limit (as $\mathrm{g} \rightarrow 0$ ) of the potential, evaluated at its minimum. Using the expression of the potential (3.22) and the expansion of the quantum dilogarithm (3.30), the classical limit is found to be

$$
V_{m, n, \xi}^{(0)}(u)=-\frac{m+n}{2 \pi} u-\frac{m+n+1}{2 \pi^{2}} \operatorname{Im}\left\{\operatorname{Li}_{2}\left(-\mathrm{e}^{u+\frac{\pi \mathrm{i}(m+1)}{m+n+1}}\right)+\operatorname{Li}_{2}\left(-\mathrm{e}^{u-\widehat{\zeta}+\frac{\pi i n}{m+n+1}}\right)\right\}+\frac{n \widehat{\zeta}}{2 \pi},
$$

where

$$
\widehat{\zeta}=\frac{2 \pi \zeta}{b} .
$$

One can check that, for $m=n=1$, the minimum of this potential occurs at

$$
\mathrm{e}^{u_{\star}}=\frac{1}{2}\left(1+\sqrt{1+4 \mathrm{e}^{\widehat{\zeta}}}\right)
$$

If we now use (3.24), we find that

$$
\begin{aligned}
& \left.\frac{\partial \mathcal{F}_{0}}{\partial \lambda_{1}}\right|_{\lambda_{j}=0}=-V_{1,1, \xi}^{(0)}\left(u_{\star}\right)-\frac{1}{6 \pi} \log \widehat{b}_{3}, \\
& \left.\frac{\partial \mathcal{F}_{0}}{\partial \lambda_{2}}\right|_{\lambda_{j}=0}=-V_{1,1, \xi}^{(0)}\left(u_{\star}\right)-\frac{1}{6 \pi} \log \widehat{b}_{0} .
\end{aligned}
$$

Thanks to (2.71), this gives a prediction for the value of the Kähler moduli at the MCL, which we will denote by $t_{i}^{(c)}, i=1,2$. In terms of

$$
\widehat{z}_{3}=\frac{1}{\widehat{b}_{3} \widehat{b}_{0}}=\mathrm{e}^{-3 \widehat{\zeta}}
$$


we find that

$$
\begin{aligned}
t_{1}^{(c)}= & t_{2}^{(c)}=-2 \log \left[\frac{1}{2}\left(1+\sqrt{\frac{4}{\widehat{z}_{3}^{1 / 3}}+1}\right)\right] \\
& -\frac{3}{\pi} \operatorname{Im}\left\{\operatorname{Li}_{2}\left(-\frac{\mathrm{e}^{2 \pi \mathrm{i} / 3}}{2}\left(1+\sqrt{\frac{4}{\widehat{z}_{3}^{1 / 3}}+1}\right)\right)+\operatorname{Li}_{2}\left(-\frac{\mathrm{e}^{\pi \mathrm{i} / 3} \widehat{z}_{3}^{1 / 3}}{2}\left(1+\sqrt{\frac{4}{\widehat{z}_{3}^{1 / 3}}+1}\right)\right)\right\} .
\end{aligned}
$$

The fact that the value of the periods at the MCL can be computed in terms of classical dilogarithms was first pointed out in [63], and further developed in [64]. As explained in $[2,19,27]$, the conjectural correspondence of $[1,27]$ allows us to explain this numbertheoretic property of the periods in many examples. The classical dilogarithm enters as a classical limit of the quantum dilogarithm involved in the kernel of the relevant operator. It would be very interesting to prove (3.41) by using the powerful techniques of [64].

\subsection{Topological string calculations}

In this section, we perform various tests of the general conjectures put forward in $[1,27]$, in the $Y^{3,0}$ geometry. In particular, we will test the validity of the conjecture (2.65), (2.69): we will check the prediction for the GW invariants (3.35) in the MCF, as well as the values of the Kähler moduli at the MCL (3.41). To do this, we review the special geometry of this model in some detail.

From the charge vectors (3.3), we can write down the Picard-Fuchs operators

$$
\begin{aligned}
& L_{1}=\theta_{21}\left(\theta_{3}-\theta_{1}\right)-z_{1} \theta_{12}\left(1+\theta_{12}\right), \\
& L_{2}=L_{1}(1 \leftrightarrow 2), \\
& L_{3}=\theta_{3}^{2}-z_{3}\left(\theta_{3}-\theta_{1}\right)\left(\theta_{3}-\theta_{2}\right) .
\end{aligned}
$$

where $\theta_{i}=z_{i} \frac{\partial}{\partial_{z_{i}}}$, and we have defined

$$
\theta_{12} \equiv 2 \theta_{2}-\theta_{1}, \theta_{21} \equiv 2 \theta_{1}-\theta_{2} .
$$

Furthermore, using the sum of the charge vectors $Q_{1}+Q_{2}+Q_{3}$, we can get an additional Picard-Fuchs operator

$$
L_{0}=\theta_{3}^{2}-z_{1} z_{2} z_{3} \theta_{12} \theta_{21},
$$

which helps eliminate the false flat coordinates. Using these Picard-Fuchs operators, we obtain in the LRF the A-periods

$$
\begin{aligned}
t_{1}= & -\log \left(z_{1}\right)+\left(-2 z_{1}+z_{2}\right)+\left(-3 z_{1}^{2}+\frac{3 z_{2}^{2}}{2}\right) \\
& +\left(-\frac{20 z_{1}^{3}}{3}+2 z_{1}^{2} z_{2}-z_{1} z_{2}^{2}+\frac{10 z_{2}^{3}}{3}\right) \\
& +\left(-\frac{35 z_{1}^{4}}{2}+8 z_{1}^{3} z_{2}-4 z_{1} z_{2}^{3}+\frac{35 z_{2}^{4}}{4}+4 z_{1}^{2} z_{2} z_{3}-2 z_{1} z_{2}^{2} z_{3}\right) \\
& +\left(-\frac{252 z_{1}^{5}}{5}+30 z_{1}^{4} z_{2}-15 z_{1} z_{2}^{4}+\frac{126 z_{2}^{5}}{5}+24 z_{1}^{3} z_{2} z_{3}-12 z_{1} z_{2}^{3} z_{3}\right)+\mathcal{O}\left(z^{6}\right), \\
t_{2}= & t_{1}\left(z_{1} \leftrightarrow z_{2}\right), \\
t_{\xi}= & t_{3}=-\log \left(z_{3}\right),
\end{aligned}
$$


as well as the B-periods

$$
\begin{aligned}
\frac{\partial F_{0}}{\partial t_{1}}= & \log ^{2}\left(z_{1}\right)+\log \left(z_{1}\right) \log \left(z_{2}\right)+\frac{1}{2} \log ^{2}\left(z_{2}\right)+\frac{2}{3} \log \left(z_{1}\right) \log \left(z_{3}\right)+\frac{1}{3} \log \left(z_{2}\right) \log \left(z_{3}\right) \\
& +\log \left(z_{1}\right)\left(3 z_{1}+\frac{9}{2} z_{1}^{2}\right)+\log \left(z_{2}\right)\left(z_{1}+z_{2}+\frac{3}{2} z_{1}^{2}+\frac{3}{2} z_{2}^{2}\right)+\log \left(z_{3}\right)\left(z_{1}+\frac{3}{2} z_{1}^{2}\right) \\
& +2 z_{1}+7 z_{1}^{2}-z_{1} z_{2}+z_{2}^{2}+\mathcal{O}\left(z^{3}\right), \\
\frac{\partial F_{0}}{\partial t_{2}}= & \frac{\partial F_{0}}{\partial t_{1}}\left(z_{1} \leftrightarrow z_{2}\right) .
\end{aligned}
$$

The discriminant of the Picard-Fuchs system is

$$
\begin{aligned}
\Delta= & -729 z_{1}^{4} z_{2}^{4} z_{3}^{3}+2187 z_{1}^{4} z_{2}^{4} z_{3}^{2}-2187 z_{1}^{4} z_{2}^{4} z_{3}+729 z_{1}^{4} z_{2}^{4}+1215 z_{1}^{3} z_{2}^{3} z_{3}^{2}-243 z_{1}^{3} z_{2}^{3} z_{3}-972 z_{1}^{3} z_{2}^{3} \\
& -27 z_{1}^{3} z_{2}^{2} z_{3}^{2}+540 z_{1}^{3} z_{2}^{2} z_{3}+216 z_{1}^{3} z_{2}^{2}-27 z_{1}^{2} z_{2}^{3} z_{3}^{2}+540 z_{1}^{2} z_{2}^{3} z_{3}+216 z_{1}^{2} z_{2}^{3}-513 z_{1}^{2} z_{2}^{2} z_{3} \\
& +270 z_{1}^{2} z_{2}^{2}+36 z_{1}^{2} z_{2} z_{3}-144 z_{1}^{2} z_{2}+16 z_{1}^{2}+36 z_{1} z_{2}^{2} z_{3}-144 z_{1} z_{2}^{2}-z_{1} z_{2} z_{3} \\
& +68 z_{1} z_{2}-8 z_{1}+16 z_{2}^{2}-8 z_{2}+1
\end{aligned}
$$

The zero locus of this discriminant defines the conifold locus. The MCL is the submanifold of the conifold locus where $\lambda_{1}=\lambda_{2}=0$. In this case, since we have a mass parameter, this is a one-dimensional complex manifold. If we fix the value of $z_{3}$, we obtain a point in the MCL which we call a maximal conifold point (MCP). As in [27], the MCP for a fixed value of $z_{3}$ corresponds to the point where the components of the conifold locus intersect transversally, see figure 1.

One ingredient we need for our calculation is the B-field. In this geometry it is given by $[28]$

$$
\boldsymbol{B}=(0,0,1)
$$

This means that in the instanton part of the periods computed above (but not in the log terms) we have to change $z_{3} \rightarrow-z_{3}$. After this change of sign, the parameter $z_{3}$ should be identified with the parameters in the operator as in (3.40). To simplify the complicated algebraic manipulations, in most of the subsequent analysis we will set $z_{3}=1$, so that $\xi=1$. The discriminant locus is plotted in figure 1. One finds out that the MCP is in that case at

$$
\left(z_{1}, z_{2}\right)=(1 / 6,1 / 6) .
$$

We can already test (3.41) by evaluating numerically the LRF A-period $t_{1}$ at this point $\left(t_{2}\right.$ has the same value as $t_{1}$ since at the MCP $z_{1}=z_{2}$ ). One finds,

$$
t_{1}(1 / 6,1 / 6,1)=1.49858 \ldots,
$$

which agrees with the prediction of (3.41). One can perform the same calculation for other values of $\xi$, and the LRF A-periods evaluated at the corresponding MCP also agree with (3.41). This is a first, highly non-trivial test of the conjectures put forward in $[1,27]$.

Next, let us compute the appropriate periods in the MCF, and then the free energies $\mathcal{F}_{0}, \mathcal{F}_{1}$, which can be compared with the predictions from the large $\hbar$ expansion of the 


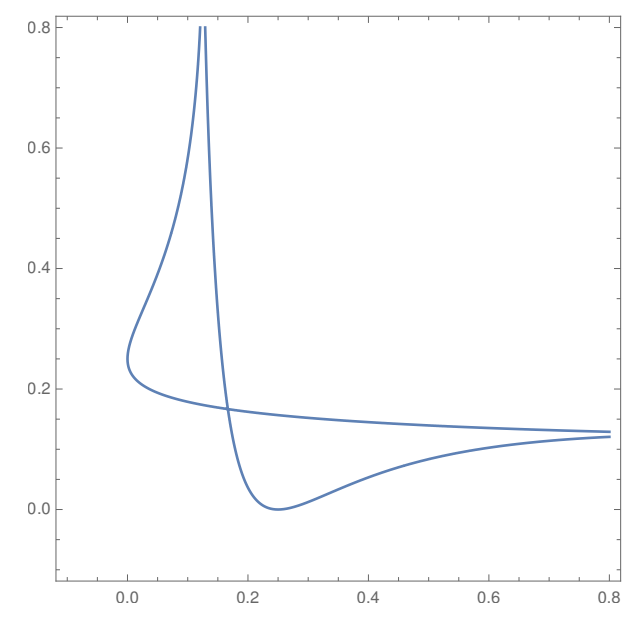

Figure 1. The discriminant locus of the geometry $Y^{3,0}$ with $z_{3}=1$. The two axes are $z_{1}$ and $z_{2}$.

fermionic traces analyzed in the previous section. Since in those computations $\xi$ is set to 1 , we fix the mass parameter $z_{3}=1$ throughout the rest of this section.

First, we look for good coordinates in the MCF, the coordinates that parametrize the neighborhood of the moduli space near the MCP. The discriminant should be factorized at first order in terms of these coordinates. We find

$$
\begin{aligned}
& z_{1}=\frac{1}{2}(3-\sqrt{5}) u_{1}+\frac{1}{2}(-3-\sqrt{5}) u_{2}+\frac{1}{6}, \\
& z_{2}=\frac{1}{2}(-3-\sqrt{5}) u_{1}+\frac{1}{2}(3-\sqrt{5}) u_{2}+\frac{1}{6} .
\end{aligned}
$$

Indeed with the new coordinates $u_{1}, u_{2}$, the discriminant is

$$
\Delta=-\frac{1125}{8} u_{1} u_{2}+\left(-\frac{135}{2} \sqrt{5} u_{1}^{3}+\frac{405}{2} \sqrt{5} u_{1}^{2} u_{2}+\frac{405}{2} \sqrt{5} u_{1} u_{2}^{2}-\frac{135}{2} \sqrt{5} u_{2}^{3}\right)+\mathcal{O}\left(u^{4}\right) .
$$

Note that the MCF coordinates $u_{1}, u_{2}$ are only defined up to scaling.

Next, we use the Picard-Fuchs equations (3.42) to solve the MCF periods. The two A-periods $\sigma_{1}, \sigma_{2}$ should be power series in $u_{1}, u_{2}$, and each of them should have leading contributions $u_{1}$ and $u_{2}$ respectively. The equations (3.42) allow three linearly independent power series solutions. Two of them have linear leading contributions, while the third one has quadratic leading contributions. The correct combinations are found by the requirement that the A-periods should vanish over the zero locus of the discriminant. In other words, expressed in terms of the MCF A-periods, the discriminant should factorize at every order. We find

$$
\begin{aligned}
\sigma_{1}= & u_{1}+\left(\frac{112 u_{1}^{2}}{5 \sqrt{5}}-\frac{12 u_{1} u_{2}}{5 \sqrt{5}}+\frac{12 u_{2}^{2}}{5 \sqrt{5}}\right)+\left(\frac{8984 u_{1}^{3}}{75}-\frac{1212}{25} u_{1}^{2} u_{2}+\frac{588}{25} u_{1} u_{2}^{2}+\frac{228 u_{2}^{3}}{25}\right) \\
& +\left(\frac{3812896 u_{1}^{4}}{1125 \sqrt{5}}-\frac{221888 u_{1}^{3} u_{2}}{125 \sqrt{5}}+\frac{1824 u_{1}^{2} u_{2}^{2}}{5 \sqrt{5}}+\frac{38112 u_{1} u_{2}^{3}}{125 \sqrt{5}}+\frac{32544 u_{2}^{4}}{125 \sqrt{5}}\right)+\mathcal{O}\left(u^{5}\right), \\
\sigma_{2}= & \sigma_{1}\left(u_{1} \leftrightarrow u_{2}\right) .
\end{aligned}
$$


The mirror map follows

$$
\begin{aligned}
u_{1}= & \sigma_{1}+\left(-\frac{112 \sigma_{1}^{2}}{5 \sqrt{5}}+\frac{12 \sigma_{1} \sigma_{2}}{5 \sqrt{5}}-\frac{12 \sigma_{2}^{2}}{5 \sqrt{5}}\right)+\left(\frac{29912 \sigma_{1}^{3}}{375}+\frac{492}{25} \sigma_{1}^{2} \sigma_{2}-\frac{348}{25} \sigma_{1} \sigma_{2}^{2}+\frac{1404 \sigma_{2}^{3}}{125}\right) \\
& +\left(-\frac{6903584 \sigma_{1}^{4}}{5625 \sqrt{5}}-\frac{843072 \sigma_{1}^{3} \sigma_{2}}{625 \sqrt{5}}+\frac{370896 \sigma_{1}^{2} \sigma_{2}^{2}}{625 \sqrt{5}}+\frac{18128 \sigma_{1} \sigma_{2}^{3}}{625 \sqrt{5}}-\frac{117376 \sigma_{2}^{4}}{625 \sqrt{5}}\right)+\mathcal{O}\left(\sigma^{5}\right), \\
u_{2}= & u_{1}\left(\sigma_{1} \leftrightarrow \sigma_{2}\right),
\end{aligned}
$$

Similar to the coordinates $u_{i}$, we have the freedom to rescale the conifold frame Aperiods $\sigma_{i}$. On the other hand, the conifold frame flat coordinates $\lambda_{i}$ that are identified with the 't Hooft parameters in the large $\hbar$ expansion of the fermionic traces can be expressed in terms of the LRF B-periods according to (2.67). The constants $b_{j}^{\mathrm{NS}}$ that appear in (2.67) are $[28]$

$$
b_{1}^{\mathrm{NS}}=b_{2}^{\mathrm{NS}}=-\frac{1}{6} .
$$

By evaluating $\lambda_{i}$ and $\sigma_{i}$ at several points in the moduli space between the large radius point (LRP) and the MCP, we find that

$$
\sigma_{i}=r_{i} \lambda_{i}, \quad i=1,2,
$$

where

$$
r_{1}=r_{2}=\frac{\pi^{2}}{3 \sqrt{3}} .
$$

This fixes the scaling of the MCF periods.

Next, we wish to compute the two B-periods, which have the form

$$
s_{i}=\lambda_{i} \log \lambda_{i}+\text { power series }\left(\lambda_{1}, \lambda_{2}\right), \quad i=1,2 .
$$

The B-periods can be solved by plugging the ansatz into the Picard-Fuchs equation, and also demanding that the two B-periods are the derivatives of a single function, namely the MCF prepotential. Expressed in terms of the MCF A-periods $\lambda_{1}, \lambda_{2}$ we find

$$
\begin{aligned}
s_{1}= & \lambda_{1} \log \left(\lambda_{1}\right)+\left(-\frac{16 \pi^{2} \lambda_{1}^{2}}{15 \sqrt{15}}+\frac{2 \pi^{2} \lambda_{1} \lambda_{2}}{\sqrt{15}}+\frac{\pi^{2} \lambda_{2}^{2}}{\sqrt{15}}\right) \\
& +\left(\frac{3464 \pi^{4} \lambda_{1}^{3}}{30375}-\frac{682 \pi^{4} \lambda_{1}^{2} \lambda_{2}}{1125}-\frac{106 \pi^{4} \lambda_{1} \lambda_{2}^{2}}{1125}-\frac{682 \pi^{4} \lambda_{2}^{3}}{3375}\right) \\
& +\left(-\frac{102848 \pi^{6} \lambda_{1}^{4}}{273375 \sqrt{15}}+\frac{131744 \pi^{6} \lambda_{1}^{3} \lambda_{2}}{50625 \sqrt{15}}-\frac{2624 \pi^{6} \lambda_{1}^{2} \lambda_{2}^{2}}{16875 \sqrt{15}}-\frac{5248 \pi^{6} \lambda_{1} \lambda_{2}^{3}}{50625 \sqrt{15}}+\frac{123808 \pi^{6} \lambda_{2}^{4}}{151875 \sqrt{15}}\right) \\
& +\mathcal{O}\left(\lambda_{i}^{5}\right) . \\
s_{2}= & s_{1}\left(\lambda_{1} \leftrightarrow \lambda_{2}\right) .
\end{aligned}
$$

The conifold frame periods also satisfy the special geometry relations; in other words

$$
\frac{\partial \mathcal{F}_{0}}{\partial \lambda_{1}}=s_{1}+\left(\alpha+\frac{1}{2}\right) \lambda_{1}+\beta \lambda_{2}-\gamma, \quad \frac{\partial \mathcal{F}_{0}}{\partial \lambda_{2}}=s_{2}+\left(\alpha+\frac{1}{2}\right) \lambda_{2}+\beta \lambda_{1}-\gamma .
$$


The coefficients $\alpha, \beta$ cannot be obtained from this method, but they are determined by the relationship (2.71) (the value of $\gamma$ is fixed by (3.39)). They involve the analytic continuation of the B-periods from the LRF to the MCF. The conjecture of $[1,27]$ gives a prediction for the values of these coefficients in terms of spectral theory. Such a prediction was tested for the resolved $\mathbb{C}^{3} / \mathbb{Z}_{5}$ orbifold in [27]. It would be interesting to test it in this example as well, as a function of the mass parameter. By integrating the right hand side of (3.59), we get the prepotential $\mathcal{F}_{0}$ in the MCF, up to an additive constant:

$$
\begin{aligned}
\mathcal{F}_{0}\left(\lambda_{1}, \lambda_{2}\right)= & \frac{1}{2} \lambda_{1}^{2} \log \left(\lambda_{1}\right)+\frac{1}{2} \lambda_{2}^{2} \log \left(\lambda_{2}\right)+\left(\frac{\alpha \lambda_{1}^{2}}{2}+\frac{\alpha \lambda_{2}^{2}}{2}+\beta \lambda_{1} \lambda_{2}\right)-\gamma\left(\lambda_{1}+\lambda_{2}\right) \\
& +\left(-\frac{16 \pi^{2} \lambda_{1}^{3}}{45 \sqrt{15}}+\frac{\pi^{2} \lambda_{1}^{2} \lambda_{2}}{\sqrt{15}}+\frac{\pi^{2} \lambda_{1} \lambda_{2}^{2}}{\sqrt{15}}-\frac{16 \pi^{2} \lambda_{2}^{3}}{45 \sqrt{15}}\right) \\
& +\left(\frac{866 \pi^{4} \lambda_{1}^{4}}{30375}+\frac{866 \pi^{4} \lambda_{2}^{4}}{30375}+\ldots\right)+\left(-\frac{102848 \pi^{6} \lambda_{1}^{5}}{1366875 \sqrt{15}}-\frac{102848 \pi^{6} \lambda_{2}^{5}}{1366875 \sqrt{15}}+\ldots\right) \\
& +\mathcal{O}\left(\lambda_{i}^{6}\right) .
\end{aligned}
$$

The coefficients of the prepotential in the second and third lines of (3.60) indeed agree with the numbers in (3.35). We proceed to the genus one standard topological string free energy in the MCF. It has the form,

$$
\mathcal{F}_{1}=-\frac{1}{12} \log \left(\Delta z_{1}^{a} z_{2}^{b}\right)-\frac{1}{2} \log \operatorname{det}\left(\frac{\partial \lambda_{i}}{\partial z_{j}}\right) .
$$

The two exponents $a$ and $b$ should be identical due to the symmetry of the geometry. Furthermore, we can use the first genus one MCF Gromov-Witten invariant in (3.35) to fix $a=8$. Plugging in the MCF mirror map (3.53), we have

$$
\begin{aligned}
\mathcal{F}_{1}= & -\frac{1}{12} \log \left(\lambda_{1} \lambda_{2}\right)+\left(\frac{16 \pi^{2} \lambda_{1}}{45 \sqrt{15}}+\frac{16 \pi^{2} \lambda_{2}}{45 \sqrt{15}}\right)+\left(-\frac{146 \pi^{4} \lambda_{1}^{2}}{30375}+\frac{533 \pi^{4} \lambda_{1} \lambda_{2}}{3375}-\frac{146 \pi^{4} \lambda_{2}^{2}}{30375}\right) \\
& +\left(-\frac{9472 \pi^{6} \lambda_{1}^{3}}{1366875 \sqrt{15}}-\frac{48256 \pi^{6} \lambda_{1}^{2} \lambda_{2}}{151875 \sqrt{15}}-\frac{48256 \pi^{6} \lambda_{1} \lambda_{2}^{2}}{151875 \sqrt{15}}-\frac{9472 \pi^{6} \lambda_{2}^{3}}{1366875 \sqrt{15}}\right)+\mathcal{O}\left(\lambda_{i}^{4}\right) .
\end{aligned}
$$

Again, the coefficients agree with all the genus one numbers in (3.35) perfectly.

\subsection{Spectral traces from Airy functions}

In this section we present a check of (2.58). On the one hand, since we have the integral kernels of the operators $\mathrm{A}_{1}, \mathrm{~A}_{2}$ associated to the $Y^{3,0}$ geometry, we can directly compute the fermionic traces $Z\left(N_{1}, N_{2} ; \hbar\right)$. In particular, the kernels $A_{1}(x, y)=\rho_{1,1, \xi}(x, y)$ and $A_{2}(x, y)$ given in (3.29) are tremendously simplified when $\hbar$ is a rational multiple of $2 \pi$. On the other hand, as already pointed in $[1,27],(2.58)$ gives a convenient way to compute $Z\left(N_{1}, N_{2} ; \hbar\right)$ from the conjectured form of the Fredholm determinant in terms of Airy functions. Let us review the Airy function method and generalize it slightly.

We first separate $J(\boldsymbol{\mu} ; \boldsymbol{\xi}, \hbar)$ into the perturbative part $J^{(\mathrm{p})}$, which is a cubic polynomial in $\mu$, and the nonperturbative part $J^{(\mathrm{np})}$, which is a power series in $\mathrm{e}^{-\mu}$. (2.58) implies that

$$
Z(\boldsymbol{N} ; \boldsymbol{\xi}, \hbar)=\int_{\mathcal{C}} \mathrm{e}^{J^{(\mathrm{p})}+J^{(\mathrm{np})}-\sum_{i=1}^{g_{\Sigma}} N_{i} \mu_{i}} \prod_{i=1}^{g_{\Sigma}} \frac{\mathrm{d} \mu_{i}}{2 \pi \mathrm{i}} .
$$




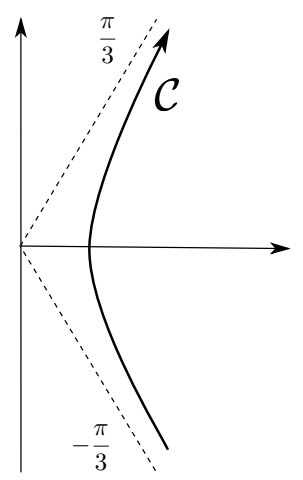

Figure 2. The integration contour $\mathcal{C}$ used in the computation of $Z(\boldsymbol{N} ; \boldsymbol{\xi}, \hbar)$.

Here the integration path $\mathcal{C}$ for each $\mathrm{d} \mu_{i}$ has been deformed so that it asymptotes to $\mathrm{e}^{ \pm \mathrm{i} \pi / 3} \infty$, as seen in figure 2. Let us ignore the nonperturbative contributions for the moment, and let us define

$$
Z^{(\mathrm{p})}(\boldsymbol{N} ; \boldsymbol{\xi}, \hbar)=\int_{\mathcal{C}} \mathrm{e}^{J^{(\mathrm{p})}-\sum_{i=1}^{g_{\Sigma}} N_{i} \mu_{i}} \prod_{i=1}^{g_{\Sigma}} \mathrm{d} \mu_{i}
$$

When $g_{\Sigma}=1$, this is nothing else but an Airy function, since the coefficients of the cubic terms are triple intersection numbers of the geometry and must therefore be real and positive. In the case of $g_{\Sigma}=2$, we can always find a linear combination of $\mu_{1,2}$

$$
\nu_{i}=\sum_{j=1}^{2} K_{i j} \mu_{j}, \quad i=1,2
$$

such that $J^{(\mathrm{p})}$ has the following form

$$
J^{(p)}=\sum_{i=1}^{2}\left(\frac{C_{i}}{3} \nu_{i}^{3}+D_{i} \nu_{i}^{2}+B_{i} \nu_{i}\right)+\zeta \nu_{1} \nu_{2}+A
$$

where the coefficient $C_{i}$ is proportional to $\hbar^{-1}$ and positive, while $D_{i}, B_{i}, A$ are functions of the mass parameters $\xi_{j}$ and $\hbar$ (the coefficient $\zeta$ should not be confused with other occurrences of the same symbol in this paper, in (2.60) and (2.75)). The Jacobian must not vanish, i.e.

$$
\left(\frac{\partial \mu_{i}}{\partial \nu_{j}}\right) \neq 0
$$

for the integral measure in (3.63) to be still meaningful after the coordinate transformation. In addition, we define $M_{i}$ by

$$
\sum_{i=1}^{2} \mu_{i} N_{i}=\sum_{i=1}^{2} \nu_{i} M_{i}
$$


If $\zeta=0$, the quadratic mixing term is absent, and $Z^{(\mathrm{p})}$ splits to a product of Airy functions

$$
\begin{aligned}
Z^{(\mathrm{p})}(\boldsymbol{N} ; \boldsymbol{\xi}, \hbar)=\left(\frac{\partial \mu_{i}}{\partial \nu_{j}}\right) \mathrm{e}^{A} & \prod_{i}\left(C_{i}\right)^{-1 / 3} \exp \left(D_{i} C_{i}^{-1}\left(M_{i}-B_{i}+\frac{2}{3} D_{i}^{2} C_{i}^{-1}\right)\right) \\
& \times \operatorname{Ai}\left(\left(C_{i}\right)^{-1 / 3}\left(M_{i}-B_{i}+D_{i}^{2} C_{i}^{-1}\right)\right) .
\end{aligned}
$$

This is the scenario discussed in [27]. Furthermore, let

$$
\mathrm{e}^{J^{(\mathrm{np})}}=\sum_{k_{i} \geqslant 0} P_{\boldsymbol{k}}(\boldsymbol{\mu} ; \boldsymbol{\xi}, \hbar) \prod_{i=1}^{n_{\Sigma}} z_{i}^{k_{i}}
$$

where $P_{\boldsymbol{k}}(\boldsymbol{\mu} ; \boldsymbol{\xi}, \hbar)$ is a polynomial in $\mu_{i}$. Then

$$
Z(\boldsymbol{N} ; \boldsymbol{\xi}, \hbar)=\sum_{k_{i} \geqslant 0}\left(\prod_{\ell} \xi_{\ell}^{-\sum_{i} k_{i} \alpha_{i \ell}}\right) P_{\boldsymbol{k}}\left(-\partial_{\boldsymbol{N}} ; \boldsymbol{\xi}, \hbar\right) \circ Z^{(\mathrm{p})}(\boldsymbol{N}+\boldsymbol{k} \cdot C ; \boldsymbol{\xi}, \hbar) .
$$

Here $P_{\boldsymbol{k}}\left(-\partial_{\boldsymbol{N}} ; \boldsymbol{\xi}, \hbar\right)$ is the differential operator obtained through replacing variable $\mu_{i}$ by $-\partial_{N_{i}}$ in the polynomial $P_{\boldsymbol{k}}(\boldsymbol{\mu} ; \boldsymbol{\xi}, \hbar)$, and we use $\circ$ to denote its action on $Z^{(\mathrm{p})}\left(\left\{N_{i}+\right.\right.$ $\left.\left.\sum_{j} k_{j} C_{j i}\right\} ; \boldsymbol{\xi}, \hbar\right)$.

If however the mixing term $\zeta \nu_{1} \nu_{2}$ is present, $Z^{(\mathrm{p})}(\boldsymbol{N} ; \boldsymbol{\xi}, \hbar)$ cannot be split to a product of conventional Airy functions. Instead we need to $\operatorname{expand} \exp \left(\zeta \nu_{1} \nu_{2}\right)$, and replace each term in the expansion by a differential operator that acts on the $Z^{(\mathrm{p})}$ with the mixing term removed, which now can be written as a product of conventional Airy functions, just like what one has done for the terms in $J^{(\mathrm{np})}$. In compact form, one finds

$$
\begin{aligned}
& Z^{(\mathrm{p})}(\boldsymbol{N} ; \boldsymbol{\xi}, \hbar) \\
= & \left(\frac{\partial \mu_{i}}{\partial \nu_{j}}\right) \mathrm{e}^{A} \exp \left(\zeta \partial_{M_{1}} \partial_{M_{2}}\right) \circ\left(\prod_{i}\left(C_{i}\right)^{-1 / 3} \exp \left(D_{i} C_{i}^{-1}\left(M_{i}-B_{i}+\frac{2}{3} D_{i}^{2} C_{i}^{-1}\right)\right)\right. \\
& \left.\operatorname{Ai}\left(\left(C_{i}\right)^{-1 / 3}\left(M_{i}-B_{i}+D_{i}^{2} C_{i}^{-1}\right)\right)\right) .
\end{aligned}
$$

The formula for computing $Z(\boldsymbol{N} ; \boldsymbol{\xi}, \hbar)$ in terms of derivatives of $Z^{(\mathrm{p})}(\boldsymbol{N} ; \boldsymbol{\xi}, \hbar)$ is the same. In actual computations, both the expansion of $\exp \left(\zeta \nu_{1} \nu_{2}\right)$ and the expansion of $\mathrm{e}^{J^{(\mathrm{np})}}$ have to be truncated, which greatly constrains the precision of the calculations one can reach. This is the situation one will encounter in section 5. We denote the orders of the two truncations by $\operatorname{deg} \zeta$ and $\operatorname{deg} z$, and call them the perturbative degree and the instanton degree respectively.

Fortunately for the geometry $Y^{3,0}$, when $\xi=1$, which is the only situation that we will consider here, the quadratic mixing term is absent. The perturbative grand potential $J^{(\mathrm{p})}$ is

$$
J^{(\mathrm{p})}(\boldsymbol{\mu} ; \hbar)=\frac{1}{12 \pi \hbar}\left(8 \mu_{1}^{3}-3 \mu_{1}^{2} \mu_{2}-3 \mu_{1} \mu_{2}^{2}+8 \mu_{2}^{3}\right)+\left(\frac{\pi}{3 \hbar}-\frac{\hbar}{12 \pi}\right)\left(\mu_{1}+\mu_{2}\right),
$$




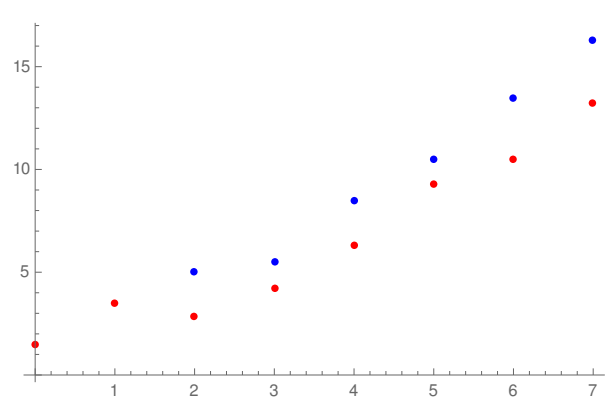

(a) $Z(1,0)$.

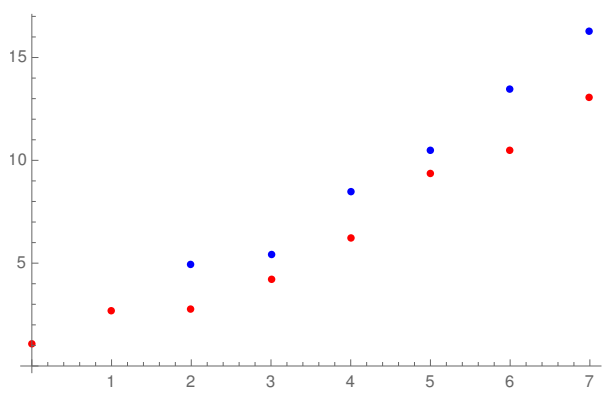

(b) $Z(2,0)$.

Figure 3. The relative matching degrees between $Z(1,0), Z(2,0)$ associated to $Y^{3,0}$ geometry $(\xi=1)$ computed with the Airy function method and the integral kernel results in (3.75), (3.76), plotted against the order of instanton corrections included in the former method. The blue and the red dots correspond to $\hbar=2 \pi$ and $\hbar=4 \pi / 3$ respectively.

and it splits with the following change of variables

$$
\left(\begin{array}{l}
\mu_{1} \\
\mu_{2}
\end{array}\right)=\left(\begin{array}{cc}
1 & \frac{7-3 \sqrt{5}}{2} \\
\frac{7-3 \sqrt{5}}{2} & 1
\end{array}\right)\left(\begin{array}{l}
\nu_{1} \\
\nu_{2}
\end{array}\right) .
$$

We will consider two particular cases. The first is the maximal supersymmetric case where $\hbar=2 \pi$. We have already seen that the integral kernel $\rho_{1,1, \xi}$ further simplifies in this case at the end of section 2.3. The first fermionic trace $Z(1,0)$ is identified with the trace of $\rho_{1,1, \xi}$, which is given by (2.99). When $\xi=1$, one has from (2.101),

$$
Z(1,0 ; \xi=1, \hbar=2 \pi)=\frac{1}{18},
$$

and by using the relation (2.29) between the fermionic traces and the spectral traces, we find in addition,

$$
Z(2,0 ; \xi=1, \hbar=2 \pi)=\frac{108-19 \sqrt{3} \pi}{1296 \sqrt{3} \pi} .
$$

We also use the Airy function method to compute these fermionic traces, including up to seven orders of instanton corrections in the modified grand potential $J(\boldsymbol{\mu} ; \xi=1, \hbar=2 \pi)$. To measure the degree of agreement between the results of the Airy function method and those using the integral kernel, we define the relative matching degree between two numbers $x$ and $y$ to be

$$
-\log _{10}\left|\frac{x-y}{y}\right|
$$

Roughly speaking it gives the number of identical digits between $x$ and $y$. We plot in figures 3 in blue dots the relative matching degrees between the Airy function results and the analytic results against the order of instanton corrections used in the former method. One finds very good agreement, and it improves consistently when more instanton contributions are included. 
The second case is $\hbar=4 \pi / 3$ so that $\mathrm{b}=\sqrt{2}$. The integral kernel also simplifies. We find

$$
\begin{aligned}
\rho_{1,1, \xi=1}(x, x) & =\frac{1}{2 \mathrm{~b} \cos (\pi / 6)} \mathrm{e}^{\frac{4}{3} \pi \mathrm{b} x} \frac{\Phi_{\mathrm{b}}\left(x+\frac{\mathrm{i}}{3} \mathrm{~b}^{-1}\right)}{\Phi_{\mathrm{b}}\left(x-\frac{\mathrm{i}}{3} \mathrm{~b}^{-1}\right)} \frac{\Phi_{\mathrm{b}}\left(x+\frac{2 \mathrm{i}}{3} \mathrm{~b}^{-1}\right)}{\Phi_{\mathrm{b}}\left(x-\frac{2 \mathrm{i}}{3} \mathrm{~b}^{-1}\right)} \\
& =\frac{1}{2 \mathrm{~b} \cos (\pi / 6)} \frac{\mathrm{e}^{\frac{4}{3} \pi \mathrm{b} x}}{1+\sqrt{3} \mathrm{e}^{2 \pi \mathrm{b}^{-1} x}+\mathrm{e}^{4 \pi \mathrm{b}^{-1} x}}
\end{aligned}
$$

so that the trace is

$$
Z(1,0 ; \xi=1, \hbar=4 \pi / 3)=\operatorname{Tr} \rho_{1,1, \xi=1}\left(\hbar=\frac{4}{3} \pi\right)=\frac{\cos \frac{\pi}{9}-\sqrt{3} \sin \frac{\pi}{9}}{3} .
$$

It is also possible to compute the double spectral trace by numerical integration, from which one gets the second fermionic trace, and it reads

$$
Z(2,0 ; \xi=1, \hbar=4 \pi / 3)=0.003565431804217254350 \ldots
$$

Similarly these fermionic traces can be computed by the Airy function method. We give the plots of the relative matching degrees in Figs 3 in red dots, with the order of instanton corrections used in the Airy function method increased up to seven. Once again one finds very good agreement between the two results.

\section{The resolved $\mathbb{C}^{3} / \mathbb{Z}_{6}$ orbifold}

In this section we study another genus two example, namely, the total resolution of the orbifold $\mathbb{C}^{3} / \mathbb{Z}_{6}$, where the action has weights $(4,1,1)$. This is an $A_{2}$ geometry studied in the first papers on local mirror symmetry [29, 41], and it engineers geometrically $\mathrm{SU}(3)$ Seiberg-Witten theory. It has also been studied in some detail in [46].

\subsection{Mirror curve and operator content}

The charge vectors of the geometry are

$$
Q^{1}=(-2,1,0,0,1,0), \quad Q^{2}=(1,-2,1,0,0,0), \quad Q^{3}=(0,0,0,1,-2,1) .
$$

We can parametrize the moduli space with the six coefficients $x_{i}, i=0, \cdots, 5$ of the mirror curve subject to three $\mathbb{C}^{*}$ scaling relations, or in terms of the Batyrev coordinates

$$
z_{1}=\frac{x_{1} x_{4}}{x_{0}^{2}}, \quad z_{2}=\frac{x_{0} x_{2}}{x_{1}^{2}}, \quad z_{3}=\frac{x_{3} x_{5}}{x_{4}^{2}} .
$$

It is convenient to set $x_{2}=x_{3}=x_{5}=1$, so that the mirror curve reads

$$
\mathrm{e}^{x}+\mathrm{e}^{y}+\mathrm{e}^{-4 x-y}+x_{4} \mathrm{e}^{-2 x}+x_{0} \mathrm{e}^{-x}+x_{1}=0 .
$$

It follows from the Newton polygon that $x_{0}, x_{1}$ are true moduli, while $x_{4}$ is a mass parameter [46]. We rename them to $\kappa_{2}, \kappa_{1}$ and $\xi$ respectively. The curve (4.3) gives then one canonical form of the curve. It is easy to see that the other canonical form is

$$
\mathrm{e}^{2 x}+\mathrm{e}^{y}+\mathrm{e}^{-y-2 x}+\xi \mathrm{e}^{-x}+\kappa_{1} \mathrm{e}^{x}+\kappa_{2}=0 .
$$


The operators associated to this geometry are obtained by Weyl quantization of the functions appearing in (4.3), (4.4) (this was already discussed in [27]):

$$
\begin{aligned}
& \mathrm{O}_{1}=\mathrm{e}^{\mathrm{x}}+\mathrm{e}^{\mathrm{y}}+\mathrm{e}^{-4 \mathrm{x}-\mathrm{y}}+\xi \mathrm{e}^{-2 \mathrm{x}}+\kappa_{2} \mathrm{e}^{-\mathrm{x}}, \\
& \mathrm{O}_{2}=\mathrm{e}^{2 \mathrm{x}}+\mathrm{e}^{\mathrm{y}}+\mathrm{e}^{-\mathrm{y}-2 \mathrm{x}}+\xi \mathrm{e}^{-\mathrm{x}}+\kappa_{1} \mathrm{e}^{\mathrm{x}} .
\end{aligned}
$$

It follows that

$$
\begin{aligned}
& \mathrm{O}_{1}^{(0)}=\mathrm{e}^{\mathrm{x}}+\mathrm{e}^{\mathrm{y}}+\mathrm{e}^{-4 \mathrm{x}-\mathrm{y}}+\xi \mathrm{e}^{-2 \mathrm{x}}, \\
& \mathrm{O}_{2}^{(0)}=\mathrm{e}^{2 \mathrm{x}}+\mathrm{e}^{\mathrm{y}}+\mathrm{e}^{-\mathrm{y}-2 \mathrm{x}}+\xi \mathrm{e}^{-\mathrm{x}} .
\end{aligned}
$$

The first operator is a perturbation of the operator $\mathrm{O}_{4,1}$, while the second operator can be regarded as a perturbation of $\mathrm{O}_{1,1}$, after rescaling the operator $\mathrm{x}$ to $\mathrm{x}^{\prime}=2 \mathrm{x}$ (this is of course equivalent to a rescaling of $\hbar$ to $\hbar^{\prime}=2 \hbar$ ). The reduced matrix (2.36) is again given by (3.16).

In order to calculate the generalized Fredholm determinant, we pick the operators (2.21). We choose $j=1$, and we denote $\mathrm{A}_{1 i}=\mathrm{A}_{i}, i=1,2$. These operators are given by

$$
\mathrm{A}_{1}=\rho_{1}^{(0)}, \quad \mathrm{A}_{2}=\mathrm{A}_{1} \mathrm{e}^{-\mathrm{x}} .
$$

Let us note that the operators (4.6) are not of the form (2.74), and the integral kernel of their inverses is not known for $\xi \neq 0$. In order to perform an analytic test of the correspondence between spectral theory and topological strings, it is convenient to set $\xi=0$. In this case,

$$
\mathrm{A}_{1}=\rho_{4,1},
$$

and the fermionic spectral traces, with $N_{1}=0$ or $N_{2}=0$, can be calculated from the matrix model $Z_{m, n}(N, \hbar)=Z_{m, n, \xi=0}(N, \hbar)$ studied in [19]. We then find,

$$
\begin{aligned}
& Z(N, 0 ; \hbar)=Z_{4,1}(N ; \hbar), \\
& Z(0, N ; \hbar)=Z_{1,1}(N ; 2 \hbar) .
\end{aligned}
$$

The 't Hooft expansion of these matrix integrals, perturbatively in $\lambda$, has been obtained in [19]. To obtain mixed traces, we need the integral kernel of $A_{2}$. We first need to convert the variables $(x, y)$ to $(p, q)$ by $(2.78)$, which specifies in this case to

$$
x=2 \pi \mathrm{b} \frac{2 p+q}{6}, \quad y=-2 \pi \mathrm{b} \frac{4 p+5 q}{6} .
$$

One finds,

$$
A_{2}\left(p, p^{\prime}\right)=\left(p\left|\rho_{4,1} \mathrm{e}^{-\mathrm{x}}\right| p^{\prime}\right)=\mathrm{e}^{-\frac{2 \pi}{3} \mathrm{~b} p} \mathrm{e}^{-\frac{\mathrm{i} \pi}{18} \mathrm{~b}^{2}} \rho_{4,1}\left(p, p^{\prime}+\frac{\mathrm{i} \pi}{6}\right) .
$$

In addition, one can use the classical potentials $V_{4,1}^{(0)}(u), V_{1,1}^{(0)}(u)$ associated to $\mathrm{O}_{4,1}, \mathrm{O}_{1,1}$ to obtain the first derivatives of $\mathcal{F}_{0}\left(\lambda_{1}, \lambda_{2}\right)$ when $\lambda_{1}=\lambda_{2}=0$ [19]. We note that these potentials can be obtained from the general perturbed potential (3.36) by sending $\widehat{\zeta}$ to $-\infty$, which corresponds to $\xi \rightarrow 0$. The evaluation of $V_{m, m}^{(0)}(u)$ at its minimum $u_{\star}$ gives [19]

$$
V_{m, n}^{(0)}\left(u_{\star}\right)=-\frac{m}{2 \pi} \log \chi_{m}-\frac{m+n+1}{2 \pi^{2}} \operatorname{Im~Li}_{2}\left(-q^{m+1} \chi_{m}\right),
$$


where

$$
\chi_{m}(q)=\frac{q^{m}-q^{-m}}{q-q^{-1}}, \quad q=\exp \left(\frac{\pi}{m+n+1}\right) .
$$

We finally obtain,

$$
\begin{aligned}
& \left.\frac{\partial \mathcal{F}_{0}}{\partial \lambda_{1}}\right|_{\lambda_{j}=0}=-V_{4,1}^{(0)}\left(u_{\star}\right)=\frac{3}{\pi^{2}} \operatorname{Im} \operatorname{Li}_{2}\left(-\mathrm{e}^{\frac{\pi \mathrm{i}}{3}}\right), \\
& \left.\frac{\partial \mathcal{F}_{0}}{\partial \lambda_{2}}\right|_{\lambda_{j}=0}=-2 V_{1,1}^{(0)}\left(u_{\star}\right)=\frac{3}{\pi^{2}} \operatorname{Im} \operatorname{Li}_{2}\left(-\mathrm{e}^{\frac{2 \pi \mathrm{i}}{3}}\right) .
\end{aligned}
$$

The factor of 2 in the second equation is due to the rescaling of $\hbar$. As in the previous example, this calculation can be related to the evaluation of the LRF A-periods at the corresponding conifold point, and we will test it in the next section.

\subsection{Topological string calculations}

We wish to perform the same tests as in the previous sections, namely, the LRF A-periods at the MCP, as well as the MCF free energies. First of all, we know that [32]

$$
\mathbf{B}=(0,0,0),
$$

so no additional signs need to be given to the Batyrev coordinates when comparing the topological string results with the operator analysis results.

From the charge vectors (4.1) we can write down the Picard-Fuchs operators

$$
\begin{aligned}
& L_{1}=\left(2 \theta_{2}-\theta_{1}\right)\left(2 \theta_{3}-\theta_{1}\right)-z_{1}\left(2 \theta_{1}-\theta_{2}+1\right)\left(2 \theta_{1}-\theta_{2}\right), \\
& L_{2}=-\theta_{2}\left(2 \theta_{1}-\theta_{2}\right)-z_{2}\left(2 \theta_{2}-\theta_{1}+1\right)\left(2 \theta_{2}-\theta_{1}\right), \\
& L_{3}=\theta_{3}^{2}-z_{3}\left(2 \theta_{3}-\theta_{1}+1\right)\left(2 \theta_{3}-\theta_{1}\right) .
\end{aligned}
$$

which annihilate the periods. Here $\theta_{i} \equiv z_{i} \partial_{z_{i}}$. Among all the A-periods, the one associated to $z_{3}$ is special. Since $z_{3}$ is a mass parameter, the corresponding flat coordinate $Q_{3}=$ $\exp \left(-t_{3}\right)$ is a rational function of $z_{3}$. In fact [46]

$$
z_{3}=\frac{Q_{3}}{\left(1+Q_{3}\right)^{2}}
$$

This relation is valid in any reference frame of the moduli space. The other two A-periods in the LRF are

$$
\begin{aligned}
& t_{1}=-\log \left(z_{1}\right)+\left(-2 z_{1}+z_{2}+z_{3}\right)+\left(-3 z_{1}^{2}+\frac{3 z_{2}^{2}}{2}+\frac{3 z_{3}^{2}}{2}\right)+\mathcal{O}\left(z^{3}\right) \\
& t_{2}=-\log \left(z_{2}\right)+\left(z_{1}-2 z_{2}\right)+\left(\frac{3 z_{1}^{2}}{2}-3 z_{2}^{2}\right)+\mathcal{O}\left(z^{3}\right)
\end{aligned}
$$


The two B-periods are

$$
\begin{aligned}
\frac{\partial F_{0}}{\partial t_{1}}= & \frac{2}{3} \log ^{2}\left(z_{1}\right)+\frac{2}{3} \log \left(z_{1}\right) \log \left(z_{2}\right)+\frac{2}{3} \log ^{2}\left(z_{2}\right)+\frac{2}{3} \log \left(z_{1}\right) \log \left(z_{3}\right)+\frac{1}{3} \log \left(z_{2}\right) \log \left(z_{3}\right) \\
& +2 \log \left(z_{1}\right) z_{1}+2 \log \left(z_{2}\right) z_{2}+\log \left(z_{3}\right)\left(z_{1}-\frac{2}{3} z_{3}\right)+2 z_{1}+\mathcal{O}\left(z^{2}\right), \\
\frac{\partial F_{0}}{\partial t_{2}}= & \frac{1}{3} \log ^{2}\left(z_{1}\right)+\frac{4}{3} \log \left(z_{1}\right) \log \left(z_{2}\right)+\frac{4}{3} \log ^{2}\left(z_{2}\right)+\frac{1}{3} \log \left(z_{1}\right) \log \left(z_{3}\right)+\frac{2}{3} \log \left(z_{2}\right) \log \left(z_{3}\right) \\
& +2 \log \left(z_{1}\right) z_{2}+4 \log \left(z_{2}\right) z_{2}+\log \left(z_{3}\right)\left(z_{2}-\frac{1}{3} z_{3}\right)+2 z_{2}+\mathcal{O}\left(z^{2}\right) .
\end{aligned}
$$

We also have, from the results in [46] (see also [32]),

$$
b_{i}^{\mathrm{NS}}=-b_{i}=-\frac{1}{6}, \quad i=1,2 .
$$

The discriminant of the Picard-Fuchs system reads in this case

$$
\begin{aligned}
\Delta= & 729 z_{1}^{4}\left(1-4 z_{3}\right)^{2} z_{2}^{4}+108 z_{1}^{3}\left(9 z_{2}-2\right)\left(4 z_{3}-1\right) z_{2}^{2}+\left(1-4 z_{2}\right)^{2} \\
& -4 z_{1}\left(36 z_{2}^{2}-17 z_{2}+2\right)+2 z_{1}^{2}\left(108\left(4 z_{3}+1\right) z_{2}^{3}-27\left(28 z_{3}-5\right) z_{2}^{2}\right. \\
& \left.+72\left(4 z_{3}-1\right) z_{2}-32 z_{3}+8\right)
\end{aligned}
$$

and it defines the conifold locus.

We want to compare now the topological string free energies in the appropriate MCF to the 't Hooft expansion of the fermionic spectral traces. Since these have been computed for $\xi=0$, the scaling limit for the mass parameter in the 't Hooft regime is no longer the one presented in (2.61). In this example, as in the local $\mathbb{F}_{2}$ model studied in [19], we want to keep $\xi=0$ fixed in this limit. This corresponds to $Q_{3}=-1$, therefore the value of $t_{3}$ is fixed to $\pm \pi \mathrm{i}$. However, this means that the scaled variable $T_{3}$ goes to zero as $\hbar \rightarrow \infty$. In other words, when computing the topological string free energy to compare with the 't Hooft limit of the spectral traces, we have to set $T_{3}=0$, which by (4.17) means setting

$$
z_{3}=\frac{1}{4}
$$

The conifold locus corresponding to this value of $z_{3}$ is plotted in figure 4 . The MCP occurs at the point of transversal intersection of the two branches of the locus, which takes place at

$$
\left(z_{1}, z_{2}\right)=\left(\frac{4}{27}, \frac{1}{4}\right)
$$

This can be tested numerically, by verifying that the conifold coordinates given in (2.67) vanish at that point.

We can now test that the prepotential, when expanded around the MCP, reproduces the expansion of the fermionic spectral traces. To compute the prepotential, we use special geometry. We choose the coordinates near the MCP to be $\rho_{1}, \rho_{2}$, which are related to $z_{1}, z_{2}$ by

$$
z_{1}=\rho_{1}+\frac{4}{27}, \quad z_{2}=\rho_{2}+\frac{1}{4} .
$$




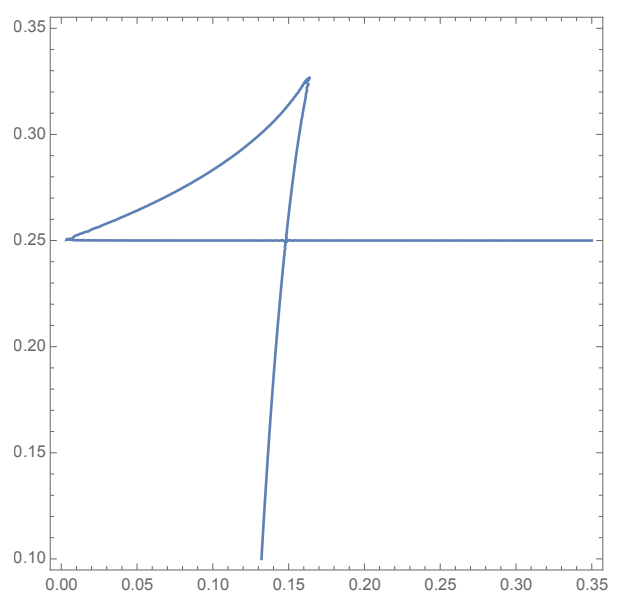

Figure 4. The discriminant locus of the resolution of local $\mathbb{C}^{3} / \mathbb{Z}_{6}$ with $z_{3}=1 / 4$. The two axes are $z_{1}$ and $z_{2}$.

The conifold A-periods $\sigma_{1}, \sigma_{2}$ are solved from the Picard-Fuchs equation, from which the mirror maps follow

$$
\begin{aligned}
& \rho_{1}=\left(\frac{2 \sigma_{1}}{27}+\frac{\sigma_{2}}{2}\right)+\left(\frac{\sigma_{1}^{2}}{6}+\frac{33 \sigma_{2}^{2}}{32}\right)+\mathcal{O}\left(\sigma_{i}^{3}\right), \\
& \rho_{2}=\frac{\sigma_{1}}{2}+\left(\frac{3 \sigma_{1}^{2}}{8}+\frac{27 \sigma_{1} \sigma_{2}}{32}\right)+\mathcal{O}\left(\sigma_{i}^{3}\right) .
\end{aligned}
$$

They satisfy the condition that the discriminant factorizes when expressed in terms of $\sigma_{1}, \sigma_{2}$. To compare these conifold A-periods with those that are identified with the 't Hooft parameters $\lambda_{1}, \lambda_{2}$, which appear in the large $\hbar$ expansion of the fermionic traces, we evaluate both $\sigma_{i}$ and $\lambda_{i}$ at several points between the LRP and the MCP. We find

$$
\begin{aligned}
\sigma_{i} & =r_{i} \lambda_{i}, & i & =1,2, \\
r_{1} & =-\frac{4 \pi^{2}}{3 \sqrt{3}}, & r_{2} & =-\frac{16 \pi^{2}}{9 \sqrt{3}} .
\end{aligned}
$$

Then we solve for the conifold B-periods $s_{1}, s_{2}$ by using again the Picard-Fuchs equations:

$$
\begin{aligned}
s_{1}= & \lambda_{1} \log \left(\lambda_{1}\right)+\left(-\frac{7 \pi^{2} \lambda_{1}^{2}}{6 \sqrt{3}}+\sqrt{3} \pi^{2} \lambda_{1} \lambda_{2}+\frac{\pi^{2} \lambda_{2}^{2}}{2 \sqrt{3}}\right) \\
& +\left(\frac{41 \pi^{4} \lambda_{1}^{3}}{54}-\frac{49}{12} \pi^{4} \lambda_{1}^{2} \lambda_{2}-\frac{1}{2} \pi^{4} \lambda_{1} \lambda_{2}^{2}-\frac{11 \pi^{4} \lambda_{2}^{3}}{108}\right)+\mathcal{O}\left(\lambda_{i}^{4}\right), \\
s_{2}= & \lambda_{2} \log \left(\lambda_{2}\right)+\left(\frac{1}{2} \sqrt{3} \pi^{2} \lambda_{1}^{2}+\frac{\pi^{2} \lambda_{1} \lambda_{2}}{\sqrt{3}}-\frac{\pi^{2} \lambda_{2}^{2}}{6 \sqrt{3}}\right) \\
& +\left(-\frac{49}{36} \pi^{4} \lambda_{1}^{3}-\frac{1}{2} \pi^{4} \lambda_{1}^{2} \lambda_{2}-\frac{11}{36} \pi^{4} \lambda_{1} \lambda_{2}^{2}+\frac{\pi^{4} \lambda_{2}^{3}}{486}\right)+\mathcal{O}\left(\lambda_{i}^{4}\right) .
\end{aligned}
$$

The conifold periods satisfy the special geometry relation

$$
\frac{\partial \mathcal{F}_{0}}{\partial \lambda_{i}}=\gamma_{i}+\sum_{j=1}^{2} \alpha_{i j} \lambda_{j}+s_{i}, \quad i=1,2,
$$


where $\gamma_{i}, \alpha_{i j}$ are constants. Integrating the right hand side, we obtain the conifold prepotential

$$
\begin{aligned}
\mathcal{F}_{0}= & \frac{1}{2} \lambda_{1}^{2} \log \left(\lambda_{1}\right)+\frac{1}{2} \lambda_{2}^{2} \log \left(\lambda_{2}\right)+q\left(\lambda_{1}, \lambda_{2}\right)+\left(-\frac{7 \pi^{2} \lambda_{1}^{3}}{18 \sqrt{3}}+\frac{1}{2} \sqrt{3} \pi^{2} \lambda_{1}^{2} \lambda_{2}+\frac{\pi^{2} \lambda_{1} \lambda_{2}^{2}}{2 \sqrt{3}}-\frac{\pi^{2} \lambda_{2}^{3}}{18 \sqrt{3}}\right) \\
& +\left(\frac{41 \pi^{4} \lambda_{1}^{4}}{216}-\frac{49}{36} \pi^{4} \lambda_{1}^{3} \lambda_{2}-\frac{1}{4} \pi^{4} \lambda_{1}^{2} \lambda_{2}^{2}-\frac{11}{108} \pi^{4} \lambda_{1} \lambda_{2}^{3}+\frac{\pi^{4} \lambda_{2}^{4}}{1944}\right)+\mathcal{O}\left(\lambda_{i}^{5}\right),
\end{aligned}
$$

where $q\left(\lambda_{1}, \lambda_{2}\right)$ is a quadratic polynomial involving $\gamma_{i}, \alpha_{i j}$ in (4.29). The coefficients of the prepotential can be compared with the predictions from the large $\hbar$ expansion of the fermionic traces, and we find a complete agreement: when $\lambda_{2}=0$, the expansion agrees precisely with the expansion obtained in [19] for the matrix model $Z_{4,1}(N, \hbar)$. When $\lambda_{1}=0$, the expansion agrees with that obtained for the matrix model $Z_{1,1}(N, \hbar)$. However, due to the rescaling of $\hbar$ in (4.9), in order to compare with the numbers presented in [19], we have to rescale $\lambda_{2} \rightarrow 2 \lambda_{2}$ and multiply the result by $1 / 4$. The crossed terms appearing in (4.30) can be also checked against the 't Hooft expansion of the mixed traces, similarly to what we did in the case of the $Y^{3,0}$ geometry. Finally, we note that, with the scaling we are using, the equation (2.71) simplifies to

$$
\frac{\partial \mathcal{F}_{0}}{\partial \lambda_{i}}=-\sum_{j=1}^{2} \frac{C_{i j}^{-1}}{2 \pi} T_{j}, \quad i=1,2 .
$$

When comparing to (4.29), we conclude that the coefficients $\gamma_{i}$ can be obtained by evaluating the LRF A-periods at the MCP. On the other hand, spectral theory gives the prediction (4.14) for their values. A numerical evaluation confirms indeed the validity of the prediction. (4.31) can be used to fix the values of the coefficients $\alpha_{i j}$ in (4.29).

As explained in [19], with the scaling we are using for the mass parameters, a test of (2.69) at next-to-leading order in $\hbar^{-1}$ involves both $\mathcal{F}_{1}$ and the derivatives of $\mathcal{F}_{0}$ w.r.t. the mass parameter. It would be interesting to perform such a test in this example.

\subsection{Spectral traces from Airy functions}

We now test the fermionic traces computed by the Airy function method against the direct integration of the integral kernel of the operators. To simplify the calculation, we set $\xi=0$, in which case the operators $\mathrm{A}_{1}, \mathrm{~A}_{2}$ reduce to $\rho_{4,1}$ and $\rho_{1,1}$ (the latter after the rescaling of $\hbar)$, and we can use the formulae (111) and (112) in [18] to compute the first two traces. The calculations are further simplified if we choose special values of $\hbar$, where the integral kernel is reduced to hyperbolic functions. For $\hbar=2 \pi$, the results of the first few traces are

$$
\begin{aligned}
& Z(1,0 ; \xi=0, \hbar=2 \pi)=\frac{1}{6 \sqrt{3}}, \\
& Z(0,1 ; \xi=0, \hbar=2 \pi)=\frac{1}{36} \\
& Z(2,0 ; \xi=0, \hbar=2 \pi)=\frac{1}{432} . \\
& Z(0,2 ; \xi=0, \hbar=2 \pi)=\frac{5}{324}-\frac{1}{12 \sqrt{3} \pi}
\end{aligned}
$$




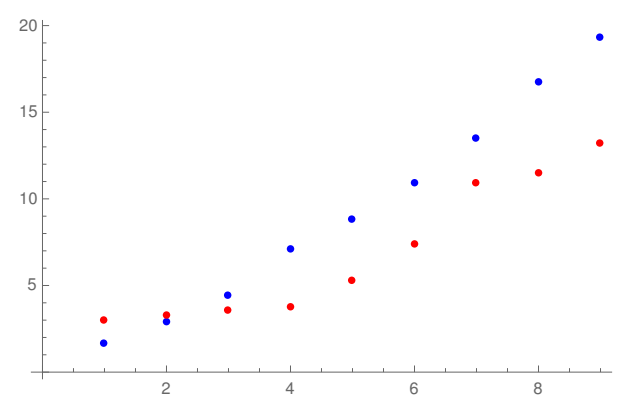

(a) $Z(1,0)$.

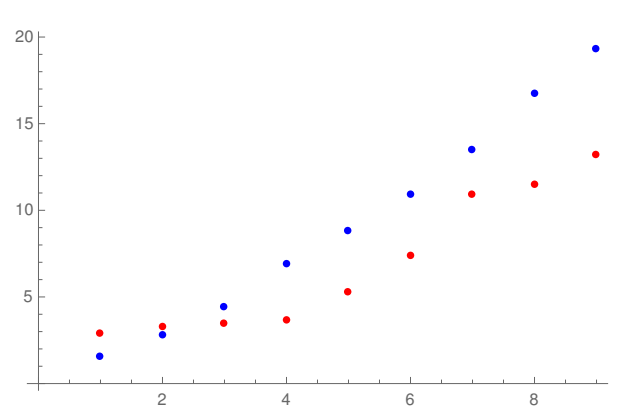

(c) $Z(2,0)$.

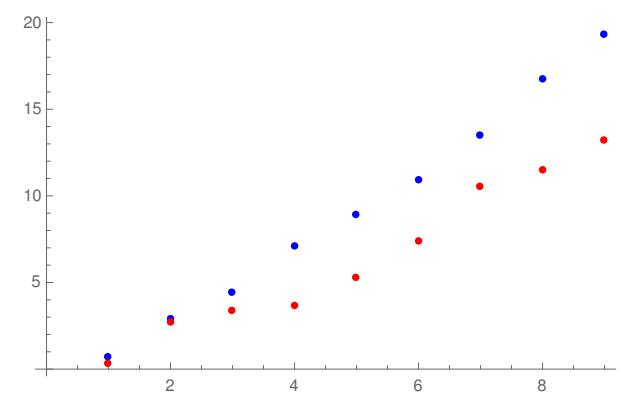

(b) $Z(0,1)$.

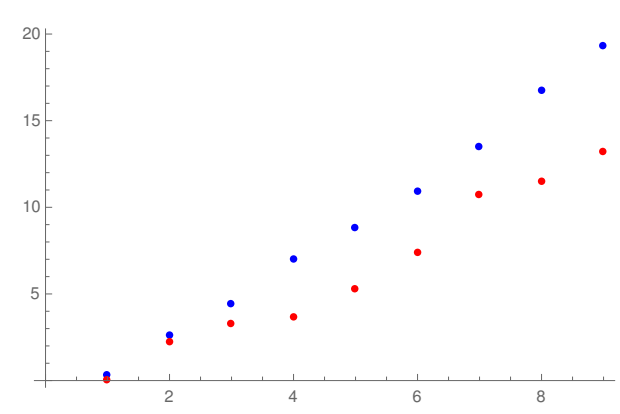

(d) $Z(0,2)$.

Figure 5. The relative matching degrees between the four fermionic traces $Z(1,0), Z(0,1), Z(2,0)$, $Z(0,2)$ associated to resolved $\mathbb{C}^{3} / \mathbb{Z}_{6}$ orbifold $(\xi=0)$ computed by the Airy function method and those by integrating the kernel, plotted against the order of instanton corrections used in the former method. The blue and red dots correspond to $\hbar=2 \pi, \pi$ respectively.

The results for $Z(0, N ; \xi=0, \hbar=2 \pi)$ can be also read from the results in [22] for the operator $\rho_{1,1}$ and $\hbar=4 \pi$. Equally simple is the case when $\hbar=\pi$, and we get

$$
\begin{aligned}
& Z(1,0 ; \xi=0, \hbar=\pi)=\frac{1}{2 \sqrt{3}}, \\
& Z(0,1 ; \xi=0, \hbar=\pi)=\frac{1}{9} \\
& Z(2,0 ; \xi=0, \hbar=\pi)=\frac{1}{36}, \\
& Z(0,2 ; \xi=0, \hbar=\pi)=-\frac{1}{81}+\frac{1}{12 \sqrt{3} \pi} .
\end{aligned}
$$

The calculation with the Airy function technique is relatively easy, as the quadratic mixing term is absent in the modified grand potential. The perturbative part of the grand potential is

$$
\begin{aligned}
J^{(\mathrm{p})}(\boldsymbol{\mu} ; \hbar)= & \frac{1}{6 \pi \hbar}\left(4 \mu_{1}^{3}-6 \mu_{1}^{2} \mu_{2}+3 \mu_{1} \mu_{2}^{2}+4 \mu_{2}^{3}\right) \\
& +\left(\frac{\pi}{3 \hbar}-\frac{\hbar}{12 \pi}\right)\left(\mu_{1}+\mu_{2}\right)-\frac{\left(\log Q_{3}\right)^{2}}{8 \pi \hbar} \mu_{1}-\frac{\left(\log Q_{3}\right)^{3}}{36 \pi \hbar} .
\end{aligned}
$$


We choose the following change of variables

$$
\left(\begin{array}{l}
\mu_{1} \\
\mu_{2}
\end{array}\right)=\left(\begin{array}{ll}
1 & \frac{1}{2} \\
0 & 1
\end{array}\right)\left(\begin{array}{l}
\nu_{1} \\
\nu_{2}
\end{array}\right),
$$

after which the modified grand potential becomes

$$
\begin{aligned}
J^{(\mathrm{p})}(\boldsymbol{\mu} ; \hbar)= & \frac{1}{12 \pi \hbar}\left(8 \nu_{1}^{3}+9 \nu_{2}^{3}\right)+\left(\frac{\pi}{6 \hbar}-\frac{\hbar}{24 \pi}\right)\left(2 \nu_{1}+3 \nu_{2}\right) \\
& -\frac{\left(\log Q_{3}\right)^{2}}{16 \pi \hbar}\left(2 \nu_{1}+\nu_{2}\right)-\frac{\left(\log Q_{3}\right)^{3}}{36 \pi \hbar} .
\end{aligned}
$$

Note that corresponding to $\xi=0$ in the operators, here we should plug in $Q_{3}=-1$, as we have discussed in the previous section. We compute the same four fermionic traces with both $\hbar=2 \pi$ and $\hbar=\pi$, with instanton corrections included up to order 9 . The relative matching degrees with the kernel results are plotted in figure 5. Yet again we see impressive agreement.

\section{$5 \quad$ Resonances and quantum spectral curves}

As we mentioned in the introduction, the operators arising from quantum mirror curves depend on the mass parameters of the geometry. One example is the operator (2.74) with $m=n=1$,

$$
\mathrm{O}_{1,1, \xi}=\mathrm{e}^{\mathrm{x}}+\mathrm{e}^{\mathrm{y}}+\mathrm{e}^{-\mathrm{x}-\mathrm{y}}+\xi \mathrm{e}^{-2 \mathrm{x}} .
$$

This operator occurs in the analysis of the quantum mirror curve for the $Y^{3,0}$ geometry considered in section 3.1. When $\xi>0$, the inverse of this operator is trace class, by an argument presented in [18]. Another example is the operator associated to the local $\mathbb{F}_{2}$ geometry,

$$
\mathrm{O}_{\mathbb{F}_{2}, \xi}=\mathrm{e}^{\mathrm{x}}+\mathrm{e}^{\mathrm{y}}+\mathrm{e}^{-2 \mathrm{x}-\mathrm{y}}+\xi \mathrm{e}^{-\mathrm{x}},
$$

which is a perturbation of the operator $\mathrm{O}_{2,1}$ (although different from (2.74)). The inverse operator is trace class when $\xi>-2$, as it can be seen by using the relation to the operator associated to local $\mathbb{F}_{0}[20,21]$.

What happens when the values of the parameters are negative? A first hint of their behavior can be obtained by considering the region of the phase space $(x, y)$ in which their classical counterparts are bounded by $\mathrm{e}^{E}$ (see for example [2] for more details on this type of argument). For the operator $\mathrm{O}_{1,1, \xi}$, we note that the value of the function

$$
\mathcal{O}_{1,1, \xi}(x, y)=\mathrm{e}^{x}+\mathrm{e}^{y}+\mathrm{e}^{-x-y}+\xi \mathrm{e}^{-2 x}, \quad(x, y) \in \mathbb{R}^{2}
$$

is unbounded from below along the directions

$$
x<y<-2 x, \quad x \rightarrow-\infty,
$$

as shown on the left in figure 6 . This suggests that the inverse operator $\rho_{2,1, \xi<0}$ is not compact. Similarly, the value of the function

$$
\mathcal{O}_{\mathbb{F}_{2}, \xi}(x, y)=\mathrm{e}^{x}+\mathrm{e}^{y}+\mathrm{e}^{-2 x-y}+\xi \mathrm{e}^{-x}, \quad(x, y) \in \mathbb{R}^{2},
$$



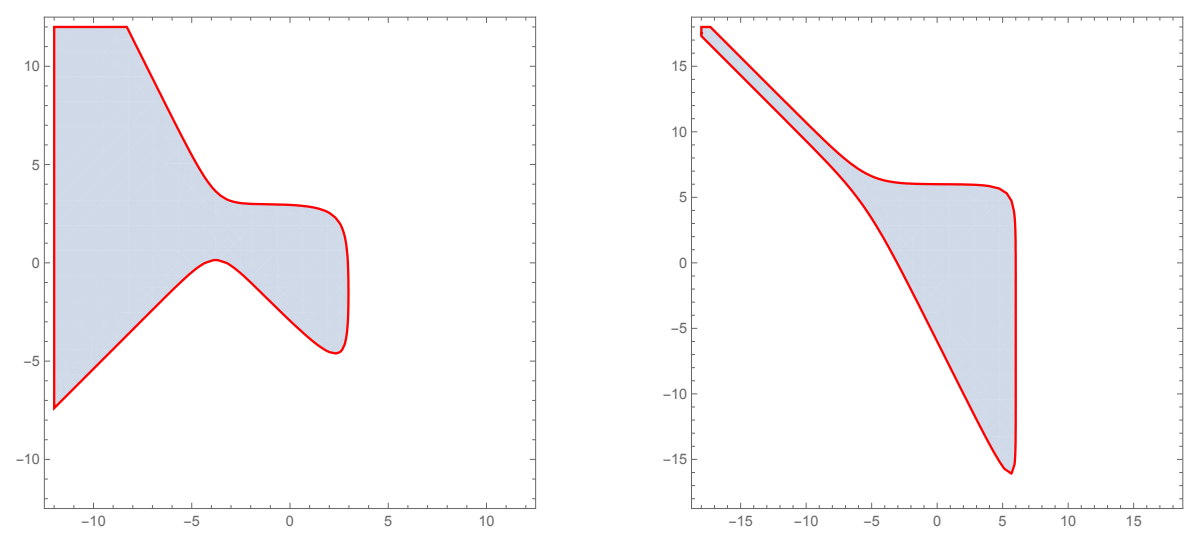

Figure 6. The regions in the $(x, y)$ plane where $\mathcal{O}_{1,1, \xi}(x, y) \leq \mathrm{e}^{E}$, with $E=3$ and $\xi=-1 / 100$ (left) and where $\mathcal{O}_{\mathbb{F}_{2}, \xi}(x, y) \leq \mathrm{e}^{E}$, with $E=6$ and $\xi=-5 / 2$ (right).

is unbounded from below along the direction

$$
y=-x, \quad x \rightarrow-\infty,
$$

when $\xi<-2$, as shown on the right in figure 6 . This suggests that $\rho_{\mathbb{F}_{2}, \xi<-2}=\mathrm{O}_{\mathbb{F}_{2}, \xi}^{-1}$ is not compact. The situation arising here is very similar to what happens in the quartic oscillator discussed in the introduction, since for negative coupling, the classical counterpart of the Hamiltonian (1.1) is also unbounded from below.

More generally, we can consider the operators (5.1) and (5.2) when the parameter $\xi$ takes arbitrary complex values. It turns out that the values of the spectral traces can be analytically continued to complex values for the mass parameter, in such a way that the conjectural formula (2.58) remains true. This is very satisfying, since after all the mass parameters in topological string theory are naturally complex numbers. As in the case of the quartic oscillator with $g<0$, we will see that, when $\xi$ is negative and the operators are non-compact, we can define a natural notion of resonance for the operators $\rho_{1,1, \xi<0}$, $\rho_{\mathbb{F}_{2}, \xi<-2}$, i.e. we will find an infinite, discrete set of complex eigenvalues. The spectral traces will be then given by sums over this resonant spectrum.

To see this in some detail, let us first look at the spectral traces of $\rho_{\mathbb{F}_{2}, \xi}$ and $\rho_{1,1, \xi}$ when $\xi>0$. These traces can be computed analytically, with the help of the Faddeev's quantum dilogarithm, especially when $\hbar=2 \pi$. For $\rho_{1,1, \xi}$, the first two traces have already been written down in (2.99) and (2.100). For convenience, we repeat the results here:

$$
\begin{aligned}
\operatorname{Tr} \rho_{1,1, \xi}(\hbar=2 \pi)= & \frac{\pi\left(2-\xi^{1 / 3}\right)+\sqrt{3} \xi^{1 / 3} \log \xi}{18 \pi\left(1-\xi^{1 / 3}+\xi^{2 / 3}\right)} \\
\operatorname{Tr} \rho_{1,1, \xi}^{2}(\hbar=2 \pi)= & -\frac{6 \sqrt{3}(1+\xi)+\pi\left(-4+4 \xi^{1 / 3}-13 \xi^{2 / 3}+6 \xi\right)}{108 \pi\left(1+\xi^{1 / 3}\right)\left(1-\xi^{1 / 3}+\xi^{2 / 3}\right)^{2}} \\
& +\frac{\xi^{2 / 3} \log \xi}{18 \sqrt{3} \pi\left(1-\xi^{1 / 3}+\xi^{2 / 3}\right)^{2}}+\frac{\xi^{2 / 3}(\log \xi)^{2}}{36 \pi^{2}\left(1+\xi^{1 / 3}\right)\left(1-\xi^{1 / 3}+\xi^{2 / 3}\right)^{2}} .
\end{aligned}
$$


For $\rho_{\mathbb{F}_{2}, \xi}$, we quote results from $[20,21]$ :

$$
\begin{aligned}
& \operatorname{Tr} \rho_{\mathbb{F}_{2}, \xi}(\hbar=2 \pi)=\frac{1}{4 \pi} \frac{\cosh ^{-1}(\xi / 2)}{\sqrt{\xi-2}}, \\
& \operatorname{Tr} \rho_{\mathbb{F}_{2}, \xi}^{2}(\hbar=2 \pi)=\frac{1}{16 \pi^{2}}\left[\left(2 \frac{\cosh ^{-1}(\xi / 2)}{\sqrt{\xi^{2}-4}}+1\right)^{2}-1-\frac{\pi^{2}}{\xi+2}\right] .
\end{aligned}
$$

One notices immediately that these functions of $\xi$ can be analytically continued to the whole complex plane with appropriate branch cuts. The traces of $\rho_{1,1, \xi}$ are multivalued functions with a branch cut starting at $\xi=0$, while the traces of $\rho_{\mathbb{F}_{2}, \xi}$ are multivalued functions of $\xi$ with a branch cut starting at $\xi=-2$. This is true for all the traces of the two operators: the traces of $\rho_{1,1, \xi}(\hbar=2 \pi)$ are rational functions of $\xi^{1 / 3}, \log \xi$, while the traces of $\rho_{\mathbb{F}_{2}, \xi}(\hbar=2 \pi)$ are always rational functions of $\cosh ^{-1}(\xi / 2), \sqrt{\xi-2}, \sqrt{\xi+2}$ (the apparent branch point at $\xi=2$ is always spurious). We now recall that the fermionic spectral traces can be recovered from the standard traces, by the standard formula

$$
Z(N)=\sum_{\left\{m_{\ell}\right\}}^{\prime} \prod_{\ell} \frac{(-1)^{(\ell-1) m_{\ell}}\left(\operatorname{Tr} \rho^{\ell}\right)^{m_{\ell}}}{m_{\ell} ! \ell^{m_{\ell}}},
$$

with the prime meaning we sum over all the partitions $\left\{m_{\ell}\right\}$ that satisfy

$$
\sum_{\ell} \ell m_{\ell}=N
$$

We can use this result to define the fermionic spectral traces as functions (with branch cuts) on the complex plane of the mass parameter $\xi$. This allows in turn to define an analytic continuation of the Fredholm determinants by using the fermionic spectral traces,

$$
\Xi_{\mathbb{F}_{2}, \xi}(\kappa)=1+\sum_{N=1}^{\infty} Z_{\mathbb{F}_{2}, \xi}(N ; \hbar) \kappa^{N}, \quad \Xi_{1,1, \xi}(\kappa)=1+\sum_{N=1}^{\infty} Z_{1,1, \xi}(N ; \hbar) \kappa^{N} .
$$

For general complex values of $\xi$, the operators are no longer of trace class, so the above formulae define just formal power series in $\kappa$. In the trace class case, the fermionic spectral traces decrease rapidly as $N$ increases, in such a way that the spectral determinants are entire functions of $\kappa$. We do not have a proof in spectral theory that this is still the case for the analytically continued fermionic traces. In order to make progress on this issue, we turn to topological string theory.

In topological string theory, the mass parameters are naturally complex. Therefore, the integral on the r.h.s. of the Airy function formula (2.58) can be computed for arbitrary complex mass parameters, in terms of topological string data. The resulting expression inherits the same branch cut structure discussed above, due to the form of the mirror map for the mass parameters. We can now ask whether the conjectural formula (2.58) remains true for arbitrary values of $\xi$, i.e. we can ask whether the analytically continued spectral traces can be still computed in terms of topological string theory. We plot in figures 7 and respectively in figures 8 the relative matching degrees between the first two spectral traces 


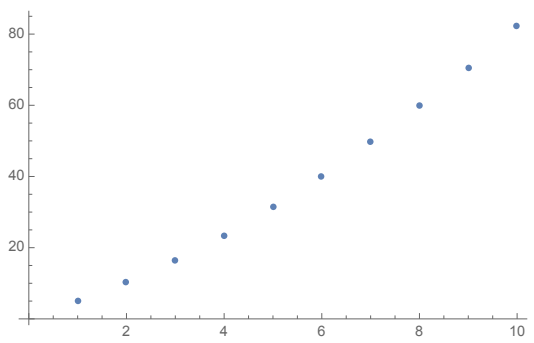

(a) $Z_{1}$.

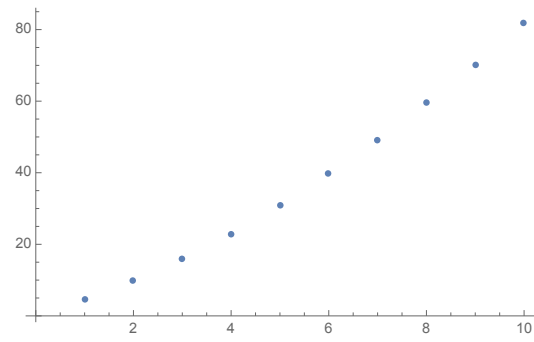

(b) $Z_{2}$.

Figure 7. The relative matching degrees between $\operatorname{Tr} \rho_{\mathbb{F}_{2}, \xi}, \operatorname{Tr} \rho_{\mathbb{F}_{2}, \xi}^{2}$ with $\xi=-3$ and $\hbar=2 \pi$ computed by the Airy function method and the analytic spectral traces in (5.8), plotted against the order of instanton corrections included in the calculation of the former.

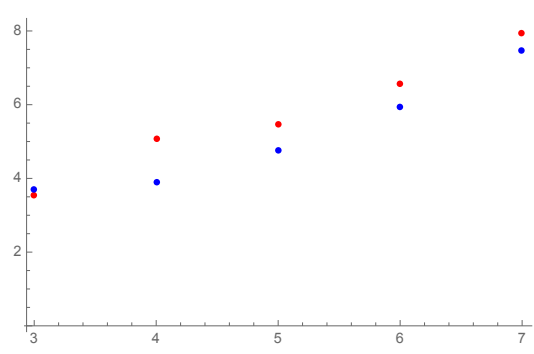

(a) Perturbative degree.

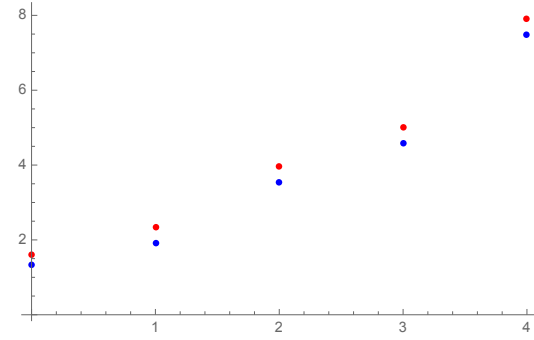

(b) Instanton degree.

Figure 8. The relative matching degrees between $\operatorname{Tr} \rho_{1,1, \xi}$ (red points), $\operatorname{Tr} \rho_{1,1, \xi}^{2}$ (blue points) with $\xi=-1$ and $\hbar=2 \pi$ computed by the Airy function method and the spectral traces in (5.7), plotted (a) against the perturbative degree, with fixed instanton degree 4, and (b) against the instanton degree, with fixed perturbative degree 7 , used in the former method.

$\operatorname{Tr} \rho, \operatorname{Tr} \rho^{2}$ computed by the Airy function method, and the results from the integral kernel, for the operators $\rho_{\mathbb{F}_{2}, \xi=-3}$ and $\rho_{1,1, \xi=-1}$ with $\hbar=2 \pi .{ }^{8}$ We find very good agreement. ${ }^{9}$

We conclude that, at least in these examples (and others we have tested) the spectral traces are still given by the r.h.s. of (2.58). It is natural to conjecture that the formula (2.58) remains true for generic values of $\xi$. Assuming this is the case, we can deduce some properties of the analytically continued spectral traces, in particular their growth rate. We will do this analysis for both geometries simultaneously, eliminating the corresponding labels. When $N$ is large, $Z(N)$ is dominated by the perturbative component $Z^{(\mathrm{p})}(N)$. Invoking the Airy function formula (3.69), roughly speaking we have

$$
|Z(N)| \sim\left|\mathrm{e}^{D \cdot C^{-1} N}\right| \operatorname{Ai}\left(C^{-1 / 3} N\right), \quad N \gg 1
$$

\footnotetext{
${ }^{8}$ In calculating these values, we have made a consistent choice of sign for the imaginary part of the multivalued functions.

${ }^{9}$ When applying the Airy function method in the case of $\rho_{1,1, \xi}$, the quadratic mixing term $\zeta \nu_{1} \nu_{2}$ does not disappear as long as $\xi \neq 1$. Therefore, as discussed in section 3.4, we have to perform both the perturbative expansion and the non-perturbative expansion. In figures 8 , the relative matching degree for $\rho_{1,1, \xi=-1}$ is plotted against not only the instanton degree but also the perturbative degree. The double expansion truncation greatly constrains the numerical precision we can reach, and in the end, as one finds in the plot, we get at most approximately $7 \sim 8$ matching digits.
} 
The coefficients $C=C(\hbar)$ and $D=D(\xi, \hbar)$ have been defined in the perturbative part of the modified grand potential $J^{(\mathrm{p})}$ in $(3.66)$. Of the two, $C(\hbar)$ is proportional to the classical triple intersection number in the CY, and for any positive real value of $\hbar$ it is always a positive constant. By using the asymptotic bound of the Airy function, we have

$$
|Z(N)| \lesssim\left|\mathrm{e}^{D \cdot C^{-1} N}\right| \frac{\mathrm{e}^{-\frac{2}{3} C^{-1 / 2} N^{3 / 2}}}{2 \sqrt{\pi} C^{-1 / 12} N^{1 / 4}} .
$$

The growth of $Z(N)$ is dominated by $\mathrm{e}^{-\frac{2}{3} C^{-1 / 2} N^{3 / 2}}$, therefore the corresponding Fredholm determinant is an entire function, regardless of the value of the mass $\xi$. The coefficient $D(\xi, \hbar)$ is a function of $\xi$, but it only affects the growth behavior at subleading order. We conclude that the functions (5.11), defined for generic $\xi$ as formal power series, are entire.

In fact, by using topological string theory, we can be more precise about the structure of the Fredholm determinant. A useful theorem in [45] shows that the entire function $\Xi_{\rho}(\kappa)$ has the infinite product form

$$
\Xi_{\rho}(\kappa)=\prod_{j=1}^{\infty}\left(1-\kappa z_{j}^{-1}\right),
$$

if the following three conditions are satisfied:

1. $\Xi_{\rho}(0)=1$;

2. $\sum_{j=1}^{\infty}\left|z_{j}\right|^{-1}<\infty$;

3. for any $\epsilon>0,\left|\Xi_{\rho}(\kappa)\right| \leqslant C(\epsilon) \exp (\epsilon|\kappa|)$.

Let us now use the information provided by topological string theory to see if these assumptions are still valid for arbitrary complex mass parameters. The first condition is satisfied by construction (the Airy function method is normalized by $Z(0)=\Xi_{\rho}(0)=1$ ). The second condition roughly speaking says the first coefficient $Z(1)$ is finite, and we have shown it by actual computation for $\rho_{\mathbb{F}_{2}, \xi}$ and $\rho_{1,1, \xi}$. The last condition puts a stricter bound on the growth of the coefficients. So we continue our estimate of $Z(N)$ from (5.12). Using the fact that asymptotically

$$
n^{1 / 2}>\alpha \log n+\beta, \forall \alpha \in \mathbb{R}_{+}, \beta \in \mathbb{R},
$$

and that

$$
\log n !<n \log n,
$$

we have

$$
\begin{aligned}
\left|\Xi_{\rho}(\kappa)\right| \leqslant \sum_{N=0}^{\infty}|Z(N)||\kappa|^{N} & \lesssim \sum_{N}\left(\left|\mathrm{e}^{D \cdot C^{-1}}\right||\kappa|\right)^{N} \mathrm{e}^{-\frac{2}{3} C^{-1 / 2} N\left(\frac{3}{2} C^{1 / 2} \log N+\beta\right)} \\
& \left.\lesssim \sum_{N} \frac{1}{N !}\left(\mathrm{e}^{\left|D \cdot C^{-1}\right|-\frac{2}{3} C^{-1 / 2} \beta}|\kappa|\right)\right)^{N} \\
& =\exp \left(\mathrm{e}^{\left|D \cdot C^{-1}\right|-\frac{2}{3} C^{-1 / 2} \beta}|\kappa|\right)
\end{aligned}
$$




\begin{tabular}{|lll|}
\hline $\mathrm{n}$ & $\operatorname{Re} \lambda$ & $\operatorname{Im} \lambda$ \\
\hline 0 & $0.08232509967018131347 \ldots$ & $-0.02921973315814942282 \ldots$ \\
1 & $0.01825649964473641110 \ldots$ & $-0.00352353844067812079 \ldots$ \\
2 & $0.00618641344745375484 \ldots$ & $-0.00092900112363282145 \ldots$ \\
\hline
\end{tabular}

Table 1. The resonant eigenvalues $\lambda \in \Delta_{\mathbb{F}_{2}, \xi}$ when $\xi=-3$ and $\hbar=2 \pi$.

Since we can tune the constant $\beta$ for the factor $\mathrm{e}^{\left|D \cdot C^{-1}\right|-\frac{2}{3} C^{-1 / 2} \beta}$ to be as (positively) small as possible, the Fredholm determinant grows in $\kappa$ more slowly than any exponential (this is in agreement with the $\exp \left((\log \kappa)^{3}\right)$ behavior noted in (109) and (110) of [2]). The last condition of the theorem is also satisfied. Note that this argument does not depend on the value of the mass parameter $\xi$, and so the infinite product form of the Fredholm determinant is valid even when the mass parameters are complex.

We conclude that, even for complex values of the mass parameter, the Fredholm determinants of the operators $\rho_{\mathbb{F}_{2}, \xi}, \rho_{1,1, \xi}$ have an infinite discrete set of complex zeros on the complex $\kappa$ plane. These zeros can be used to define the sets,

$$
\begin{aligned}
& \Delta_{\mathbb{F}_{2}, \xi}=\left\{-z_{j}^{-1}: \Xi_{\mathbb{F}_{2}, \xi}\left(z_{j}\right)=0\right\}, \\
& \Delta_{1,1, \xi}=\left\{-z_{j}^{-1}: \Xi_{1,1, \xi}\left(z_{j}\right)=0\right\} .
\end{aligned}
$$

As a concrete example, we list in table 1 the first few entries (sorted by decreasing real components) of the set $\Delta_{\mathbb{F}_{2}, \xi}$ for $\xi=-3$ and $\hbar=2 \pi$. When up to order nine instanton corrections are used in the computation of the Fredholm determinant, these entries have up to 70 stabilized digits.

When the value of $\xi$ is such that the corresponding operators are of trace class, the sets (5.18) are simply the spectra of the operators. When the operators are not compact, we regard these values as resonances, or resonant eigenvalues, of the operators. They are indeed natural analytic continuations of the eigenvalues occurring in the trace class case. In addition, one can verify that the resonant eigenvalues obtained in this way (like those listed in table 1) agree with the solutions of the quantization conditions of $[1,28,32]$ for complex values of $\xi$. It would be very interesting to see if these resonances can be obtained directly from the operators, by using generalizations of the complex dilatation techniques that are so powerful for Schrödinger operators.

\section{Quantum mirror curves and cluster integrable systems}

We defined in section 2.1 the quantum mirror curve as an operator obtained by quantizing the mirror curve to a toric CY threefold $X$ :

$$
\mathrm{W}_{X, i}=\mathrm{O}_{i}+\kappa_{i}=\mathrm{O}_{i}^{(0)}+\sum_{j} \kappa_{j} \mathrm{P}_{i j}
$$

This can be regarded as a tool to encode the operators $\mathrm{O}_{i}$ or $\mathrm{O}_{i}^{(0)}$, which are usually the three-term operators $\mathrm{O}_{m, n}$ or their perturbations. There is another spectral problem that 
can be associated to a toric CY manifold. Goncharov and Kenyon [31] assign to every $2 \mathrm{~d}$ convex Newton polygon $\mathcal{N}$ an integrable system called cluster integrable system. The number of Poisson commuting Hamiltonians $H_{i}\left(q_{k}, p_{k}\right)$ is the same as the number $g_{\Sigma}$ of inner integral vertices in $\mathcal{N}$. If we choose $\mathcal{N}$ to be the $2 \mathrm{~d}$ support of the toric fan of a toric CY threefold $X$, it turns out that the spectral curve of the classical integrable system coincides with the mirror curve of topological string theory on $X$. The $g_{\Sigma}$ true moduli are identified with the conserved Hamiltonians, while the $r_{\Sigma}$ mass parameters are identified with the Casimir parameters, up to signs.

As an example, let us revisit the $Y^{N, 0}$ geometry. By setting $q=0$ in the mirror curve for the general $Y^{N, q}$ geometry in (3.1), we get the mirror curve to the $Y^{N, 0}$ geometry ${ }^{10}$

$$
a_{1} \mathrm{e}^{y}+a_{2} \mathrm{e}^{N x-y}+\sum_{i=0}^{N} b_{i} \mathrm{e}^{i x}=0 .
$$

The mirror curve has genus $g_{\Sigma}=N-1$, corresponding to the true moduli $b_{i}, i=1, \ldots, N-$ 1. The other coefficients $a_{1}, a_{2}, b_{0}, b_{N}$ are mass parameters, while the $\mathbb{C}^{*}$ actions reduce their degrees-of-freedom to one. The associated cluster integrable system is nothing else but the relativistic, periodic $\widehat{A}_{N-1}$ Toda lattice [65-67]. The spectral curve is (6.2) with the following identification

$$
a_{1}=a_{2}=R^{N}, \quad b_{i}=(-1)^{N-i} H_{N-i}, \quad i=0,1, \ldots, N,
$$

where $H_{0}=H_{N}=1$ are trivial. Indeed the true moduli are identified with the non-trivial Hamiltonians $H_{i}, i=1, \ldots, N-1$, while the mass parameter is identified with the only Casimir parameter $R$. Let us now follow the convention in section 3 , rename the true moduli $b_{i}$ by $\kappa_{N-i}$ for $i=1, \ldots, N-1$, the mass parameter $b_{0}$ by $\xi$, and set $a_{1}, a_{2}, b_{N}$ to 1. After proper scaling, the identification (6.3) can be written as

$$
\xi=(-1)^{N} R^{-N}, \quad \kappa_{i}=(-1)^{i} R^{-i} H_{i}, \quad i=1, \ldots, N-1 .
$$

Furthermore, the Batyrev coordinates $z_{i}$ can also be expressed in terms of $H_{i}$ and $R$, and they read

$$
\begin{aligned}
z_{i} & =\frac{H_{i-1} H_{i+1}}{H_{i}^{2}}, \quad i=1, \ldots, N-1, \\
z_{N} & =(-1)^{N} R^{2 N} .
\end{aligned}
$$

It is straightforward to quantize the classical cluster integrable system. The dynamic variables $p_{k}, q_{k}$ are promoted to operators subject to the usual commutation relation

$$
\left[\mathrm{q}_{i}, \mathrm{p}_{j}\right]=\mathrm{i} \hbar \delta_{i, j}
$$

The $g_{\Sigma}$ Hamiltonian functions $H_{i}\left(p_{k}, q_{k}\right)$ are accordingly promoted to $g_{\Sigma}$ mutually commuting Hamiltonian operators

$$
\left[\mathrm{H}_{i}\left(\mathrm{p}_{k}, \mathrm{q}_{k}\right), \mathrm{H}_{j}\left(\mathrm{p}_{k}, \mathrm{q}_{k}\right)\right]=0 .
$$

\footnotetext{
${ }^{10}$ In contrast to (3.1), we use the symbol $y$ in place of $p$ since the latter has been reserved as the momentum variable of the underlying integrable system.
} 
Solving the quantum cluster integrable system means finding the spectra and eigenfunctions of these Hamiltonians. In $[28,32]$ it was conjectured that the spectra can be obtained from exact quantization conditions, akin to those obtained in [4] for the Toda lattice, and involving topological string data. It turns out that, when $g_{\Sigma}=1$, the single Hamiltonian of the cluster integrable system is precisely the (single) operator $\mathrm{O}$ obtained from the mirror curve. The quantization condition of $[28,32]$ reduces in that case to the one proposed in [33], which has been checked to be equivalent to the vanishing of the Fredholm determinant of [1].

When $g_{\Sigma}>1$, the two spectral problems associated to a toric CY involve two very different sets of operators. In particular, when mass parameters/Casimir parameters are present, sometimes the Hamiltonian operators $H_{i}$ and the operators $\mathrm{O}_{i}^{(0)}$ have real positive spectrum in different regions of the parameter space. This is the most conspicuous in the example of the $Y^{N, 0}$ geometry/ $\widehat{A}_{N-1}$ Toda lattice when $N$ is odd. In the integrable system interpretation, the Casimir $R$ has to be positive for the Hamiltonians $\mathrm{H}_{i}$ to have a positive real spectrum, while in the operator interpretation, the mass parameter $\xi$ should be non-negative for the operators $\mathrm{O}_{1}^{(0)}$ in $(3.8)$ to have a positive spectrum bounded from below. The dictionary (6.4) dictates that the two scenarios are mutually exclusive.

In spite of these differences, it turns out that, even in the higher genus case, both spectral problems are closely related. In particular, the spectra of the Hamiltonians of the cluster integrable system are located in the zero locus of the generalized Fredholm determinant $\Xi_{X}(\boldsymbol{\kappa} ; \boldsymbol{\xi}, \hbar)$. Let us give a heuristic argument for this. First, we set the Casimirs $R_{i}$ to positive values, as required by the integrable system interpretation. As we have seen in section 5 , even if the corresponding mass parameters are negative, we can analytically continue the conjectural Fredholm determinant $\Xi_{X}(\boldsymbol{\kappa} ; \boldsymbol{\xi}, \hbar)$ constructed from topological string amplitudes into this region of the parameter space, and interpret it as the Fredholm determinant in terms of the resonant eigenvalues. Let us now write the quantum mirror curve as

$$
\mathrm{W}_{X, i}=\mathrm{O}_{i}^{(0)}\left(1+\sum_{j=1}^{g_{\Sigma}} \kappa_{j} \mathrm{~A}_{i j}\right) .
$$

If the generalized spectral determinant vanishes, i.e. if

$$
\operatorname{det}\left(1+\sum_{i=1}^{g_{\Sigma}} \kappa_{j} \mathrm{~A}_{i j}\right)=0
$$

and assuming that $\mathrm{O}_{i}^{(0)}$ has a trivial kernel, there should be a state $|\Psi\rangle$ annihilated by the quantum mirror curve: $W_{X, i}|\Psi\rangle=0$. We can interpret this condition as a quantum Baxter equation for the cluster integrable system, arising from the quantization of the spectral curve. The values of the quantum Hamiltonians must be such that this equation is satisfied. Therefore, the vanishing of the generalized spectral determinant gives one constraint for the spectra of the quantum integrable system. In order to find the precise spectra, one needs $g_{\Sigma}-1$ additional conditions.

In [32] it was noted, in a genus two example, that one can impose an additional condition to determine the spectrum, involving the generalized Fredholm determinant but in terms of "rotated" variables. This observation has been extended in [34], where it 


\begin{tabular}{|ccccc|}
\hline$\left(n_{1}, n_{2}\right)$ & $\operatorname{Re} \Xi$ & $\operatorname{Im} \Xi$ & slope Re $\Xi=0$ & slope $\operatorname{Im} \Xi=0$ \\
\hline$(0,0)$ & $-3.70 \times 10^{-8}$ & $-1.24 \times 10^{-7}$ & -2.846040 & -2.846038 \\
$(1,0)$ & $6.76 \times 10^{-6}$ & $-4.46 \times 10^{-6}$ & -6.920725 & -6.920736 \\
\hline
\end{tabular}

Table 2. Values of $\operatorname{Re} \Xi\left(H_{1}, H_{2}\right)$ and $\operatorname{Im} \Xi\left(H_{1}, H_{2}\right)$ of relativistic $\widehat{A}_{2}$ Toda lattice $(\hbar=2 \pi, R=1)$ when the eigenvalues of $\mathrm{H}_{1}, \mathrm{H}_{2}$ are plugged in, as well as the slopes of the two curves $\operatorname{Re} \Xi\left(H_{1}, H_{2}\right)=$ 0 and $\operatorname{Im} \Xi\left(H_{1}, H_{2}\right)=0$ at the points corresponding to the eigen-energies on the $\left(H_{1}, H_{2}\right)$ plane.

has been proposed to use the vanishing of $g_{\Sigma}-1$ additional functions, obtained from the generalized Fredholm determinant of [27] but with different choices of the B-field.

In some cases one can consider much simpler conditions, namely, that the eigenvalues of the Hamiltonians should all be real and positive. We will now use the example of the $Y^{3,0}$ geometry/relativistic $\widehat{A}_{2}$ Toda lattice to demonstrate that, in genus two, the spectrum of the cluster integrable system can be sometimes obtained from the vanishing equation of the Fredholm determinant, together with the positive reality condition.

We use the dictionary between the geometric moduli and the quantities of the integrable system in (6.3) specified to $N=3$, and the $\mathbf{B}$-field given by (3.48). To simplify the calculation, we consider $\hbar=2 \pi$, and choose $\xi=\mathrm{e}^{\mathrm{i} \pi}=-1$ or equivalently $R=1$ in order to compare with the results in [28]. Then the computation of the coefficients $Z(\boldsymbol{N} ; \boldsymbol{\xi}, \hbar)$ is just a continuation of that at the end of section 5 . Limited by computer capacities, we can reach at most the truncation at the perturbative degree 7 and the instanton degree 4 , which as one finds in the precision plots figures 8 limits the number of accurate digits of the results of $Z(\boldsymbol{N} ; \boldsymbol{\xi}, \hbar)$ to about seven or eight.

We computed $Z\left(N_{1}, N_{2} ; \xi=\mathrm{e}^{\mathrm{i} \pi}, \hbar=2 \pi\right)$ up to $N_{1}+N_{2}=10$. Since these traces have complex values, the vanishing equation of the Fredholm determinant together with the positive reality conditions of $H_{1}, H_{2}$ is equivalent to two equations, the vanishing of both the real part and the imaginary part of the Fredholm determinant. To check that these two equations are enough to reproduce the spectrum of the $\widehat{A}_{2}$ Toda lattice in [28], we construct the real and the imaginary determinants

$$
\begin{aligned}
& \operatorname{Re} \Xi\left(H_{1}, H_{2}\right) \equiv \operatorname{Re} \Xi\left(H_{1}, H_{2} ;-1,2 \pi\right)=\sum_{N_{1}, N_{2}} \operatorname{Re} Z\left(N_{1}, N_{2} ;-1,2 \pi\right)\left(-H_{1}\right)^{N_{1}} H_{2}^{N_{2}}, \\
& \operatorname{Im} \Xi\left(H_{1}, H_{2}\right) \equiv \operatorname{Im} \Xi\left(H_{1}, H_{2} ;-1,2 \pi\right)=\sum_{N_{1}, N_{2}} \operatorname{Im} Z\left(N_{1}, N_{2} ;-1,2 \pi\right)\left(-H_{1}\right)^{N_{1}} H_{2}^{N_{2}} .
\end{aligned}
$$

We expect both of them to vanish when the eigenvalues of $\mathrm{H}_{1}, \mathrm{H}_{2}$ are plugged in. Indeed when the eigenvalues at levels $\left(n_{1}, n_{2}\right)=(0,0),(1,0)$, which are computed in [28] and reproduced in the last rows of tables 3 , are plugged in, the absolute values of $\operatorname{Re} \Xi\left(H_{1}, H_{2}\right)$ and $\operatorname{Im} \Xi\left(H_{1}, H_{2}\right)$, as seen in table 2 , are within the error margins of the Fredholm determinant estimated from the precisions of the fermionic traces, thus it is consistent with the previous statement. We also plot in figures 9 the curves $\operatorname{Re} \Xi\left(H_{1}, H_{2}\right)=0$ and $\operatorname{Im} \Xi\left(H_{1}, H_{2}\right)=0$ in the real plane of $\left(H_{1}, H_{2}\right)$, and find that the points corresponding to the eigen-energies at $\left(n_{1}, n_{2}\right)=(0,0),(1,0)$ lie on both curves. 


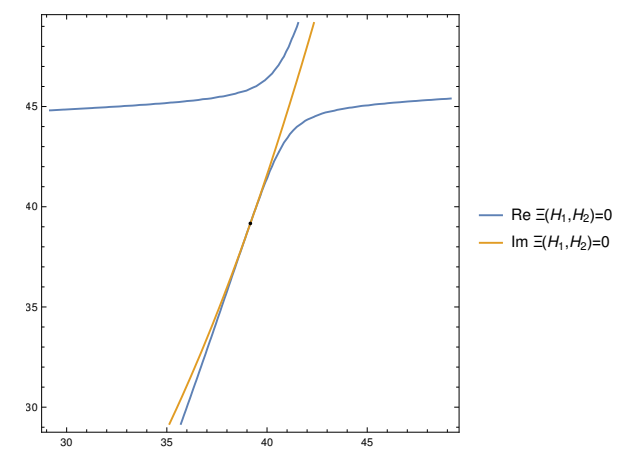

(a) $\left(n_{1}, n_{2}\right)=(0,0)$.

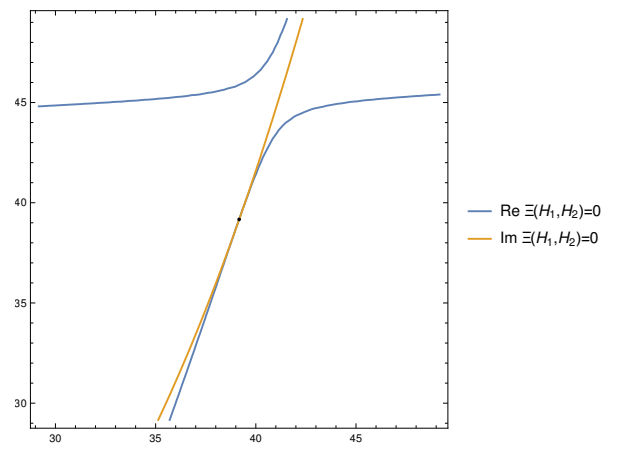

(b) $\left(n_{1}, n_{2}\right)=(1,0)$.

Figure 9. The two curves $\operatorname{Re} \Xi\left(H_{1}, H_{2}\right)=0$ and $\operatorname{Im} \Xi\left(H_{1}, H_{2}\right)=0$ on the $\mathbb{R}^{2}$ plane parametrized by $H_{1}, H_{2}$ of relativistic $\widehat{A}_{2}$ Toda lattice $(\hbar=2 \pi, R=1)$, zoomed in around the points (black) corresponding to the eigen-energies at levels $(0,0)$ and $(1,0)$.

In practice, computing the spectrum of $\widehat{A}_{2}$ Toda lattice from the double vanishing equations of $\operatorname{Re} \Xi\left(H_{1}, H_{2}\right)$ and $\operatorname{Im} \Xi\left(H_{1}, H_{2}\right)$ may not be preferable, as the two curves $\operatorname{Re} \Xi\left(H_{1}, H_{2}\right)=0$ and $\operatorname{Im} \Xi\left(H_{1}, H_{2}\right)=0$ intersect tangentially at the points of eigenenergies, as seen in figures 9 . This is confirmed by the numerical calculation of the slopes of the two curves at the points of eigen-energies, as seen in table 2. Here we compute the slope $k_{f}\left(H_{1}, H_{2}\right)$ of a curve $f\left(H_{1}, H_{2}\right)=0$ by

$$
k_{f}\left(H_{1}, H_{2}\right) \equiv \frac{\mathrm{d} H_{2}\left(H_{1} ; f\right)}{\mathrm{d} H_{1}}=-\frac{\partial_{H_{1}} f\left(H_{1}, H_{2}\right)}{\partial_{H_{2}} f\left(H_{1}, H_{2}\right)} .
$$

Therefore starting from the Fredholm determinant, we can use any two of the following three equations to compute the spectrum of $\widehat{A}_{2}$ quantum Toda lattice

$$
\begin{aligned}
& \operatorname{Re} \Xi\left(H_{1}, H_{2}\right)=0, \\
& \operatorname{Im} \Xi\left(H_{1}, H_{2}\right)=0 \text {, } \\
& k_{\operatorname{Re} \Xi}\left(H_{1}, H_{2}\right)=k_{\operatorname{Im} \Xi}\left(H_{1}, H_{2}\right) \text {. }
\end{aligned}
$$

As seen in table 3 , all three combinations give solutions consistent with the eigen-energies given in [28], while the two combinations including the identical slope equation provide solutions with better precision.

\section{Conclusions and discussion}

In this paper we continued the program started in [1, 27], and we studied the spectral theory of trace-class operators associated to mirror curves of toric CY threefolds. We focused on two geometries with genus two mirror curves and nontrivial mass parameters: the $Y^{3,0}$ geometry, and the resolved $\mathbb{C}^{3} / \mathbb{Z}_{6}$ orbifold. We tested various conjectures put forward in $[1,27]$. In particular, we checked the expression (2.58) for the fermionic spectral traces in various cases, and we verified that their 't Hooft expansion reproduces the free energies of the standard topological string in the so-called maximal conifold frame. We 


\begin{tabular}{|ccll|}
\hline$\left(n_{1}, n_{2}\right)$ & & $H_{1}$ & $H_{2}$ \\
\hline \multirow{4}{*}{$(0,0)$} & $\operatorname{Re} \Xi=0, \operatorname{Im} \Xi=0$ & $39.1604 \ldots$ & $39.1468 \ldots$ \\
& $\operatorname{Re} \Xi=0, k_{\operatorname{Re} \Xi}=k_{\operatorname{Im} \Xi}$ & $39.167844 \ldots$ & $39.167912 \ldots$ \\
& $\operatorname{Im} \Xi=0, k_{\operatorname{Re} \Xi}=k_{\operatorname{Im} \Xi}$ & $39.167834 \ldots$ & $39.167873 \ldots$ \\
\cline { 2 - 4 } & numerical & $39.16781907 \ldots$ & $39.16781907 \ldots$ \\
\multirow{2}{*}{$(1,0)$} & $\operatorname{Re} \Xi=\operatorname{Im} \Xi=0$ & $61.82 \ldots$ & $151.41 \ldots$ \\
& $\operatorname{Re} \Xi=0, k_{\operatorname{Re} \Xi}=k_{\operatorname{Im}} \Xi$ & $61.9680 \ldots$ & $152.3712 \ldots$ \\
& $\operatorname{Im} \Xi=0, k_{\operatorname{Re} \Xi}=k_{\operatorname{Im}} \Xi$ & $61.9700 \ldots$ & $152.3858 \ldots$ \\
\cline { 2 - 4 } & numerical & $61.966419 \ldots$ & $152.359672 \ldots$ \\
\hline
\end{tabular}

Table 3. The eigen-energies of $\mathrm{H}_{1}, \mathrm{H}_{2}$ of relativistic quantum $\widehat{A}_{2}$ Toda lattice $(\hbar=2 \pi, R=1)$ computed from the Fredholm determinant, using any two of the combinations of (6.12). The numerical results are quoted from [28].

were also able to write down the integral kernel of a large class of perturbations of the three-termed operator $\mathrm{O}_{m, n}$ studied in [18], and this allowed us to perform precision tests of the conjectures in the case of the $Y^{3,0}$ geometry.

We studied certain perturbations of the $\mathrm{O}_{m, n}$ operators in which the mass parameters no longer satisfy the relevant positivity conditions. As a consequence, the resulting operators are not in general of trace class. Using the correspondence with topological string theory, we obtained an analytic continuation of the Fredholm determinant. This in turn allowed us to define an infinite set of complex valued "resonant" eigenvalues of the operators, in analogy to what happens in ordinary quantum mechanics.

We also explored the connection between the spectral theory of quantum mirror curves, and the cluster integrable systems arising from toric CY threefolds. We showed that, in some cases, when the spectral curve is of genus two, the spectrum of the integrable system can be obtained from the generalized Fredholm determinant, after imposing positive reality conditions for the spectra.

There are many important questions remaining. For example, in our discussion of the resonant eigenvalues associated to mirror curves, we used an analytic continuation of the spectral traces and the Fredholm determinant. This is equivalent to a continuous deformation of the operator in the complex plane, which is one of the key ideas underlying the rigorous definition of resonances for Schrödinger operators (see for example [39]). It would be interesting to put our definition of resonances in quantum spectral curves on a rigorous footing. More pragmatically, one would like to calculate the resonant eigenvalues directly from the operator, as in the method of complex dilatation in ordinary quantum mechanics.

Another important open problem is to find the eigenfunctions of the quantum mirror curves. A general strategy to address this issue, extending the ideas of [1], has been recently put forward in [68]. ${ }^{11}$ This has led to exact formulae for the eigenfunctions in the

\footnotetext{
${ }^{11}$ See $[69,70]$ for other attempts to obtain the eigenfunctions.
} 
maximally supersymmetric case of local $\mathbb{F}_{0}$. However, much more work is needed in order to have concrete results for general geometries, and in particular for the higher genus case.

Of course, the key puzzle is the origin of the conjectured form of the Fredholm determinant in terms of topological string free energies. Recently, this has been achieved when the $\mathrm{CY}$ is local $\mathbb{F}_{0}$, in a certain $4 \mathrm{~d}$ limit [24], by showing that both sides of the conjectural formula (2.56) are solutions to a certain Painlevé equation. It would be interesting to generalize this approach to the $5 \mathrm{~d}$ case. In particular, the ubiquitous presence of mass parameters in toric CY threefolds indicates a certain hierarchy of Painlevé difference equations probably related to [71].

Finally, there are still some mysteries concerning the relationship between the Fredholm theory of quantum mirror curves and the quantization conditions for cluster integrable systems. The compatibility between both approaches, explored in detail in [33, 34], requires an intriguing relationship between the refined and the conventional topological free energies, as well as a set of B-fields providing additional vanishing functions on moduli space. This set has been found in many examples in a rather ad hoc manner. It would be interesting to give a first-principle explanation for the existence of this set, and to understand better the relationships between the free energies. Work in this direction will appear in [72].

\section{Acknowledgments}

We would like to thank Gian-Michele Graf, Alba Grassi, Yasuyuki Hatsuda, Rinat Kashaev, Amir-Kian Kashani-Poor, Thorsten Schimannek and Szabolcs Zakany for valuable discussions. SC and MM are supported in part by the Fonds National Suisse, subsidies 200021-156995 and 200020-141329, and by the NCCR 51NF40-141869 "The Mathematics of Physics" (SwissMAP). JG is supported by the grant ANR-13-BS05-0001.

Open Access. This article is distributed under the terms of the Creative Commons Attribution License (CC-BY 4.0), which permits any use, distribution and reproduction in any medium, provided the original author(s) and source are credited.

\section{References}

[1] A. Grassi, Y. Hatsuda and M. Mariño, Topological Strings from Quantum Mechanics, Annales Henri Poincaré 17 (2016) 3177 [arXiv: 1410.3382] [INSPIRE].

[2] M. Mariño, Spectral Theory and Mirror Symmetry, arXiv:1506.07757 [INSPIRE].

[3] M. Aganagic, R. Dijkgraaf, A. Klemm, M. Mariño and C. Vafa, Topological strings and integrable hierarchies, Commun. Math. Phys. 261 (2006) 451 [hep-th/0312085] [InSPIRE].

[4] N.A. Nekrasov and S.L. Shatashvili, Quantization of Integrable Systems and Four Dimensional Gauge Theories, arXiv:0908.4052 [INSPIRE].

[5] A. Mironov and A. Morozov, Nekrasov Functions and Exact Bohr-Zommerfeld Integrals, JHEP 04 (2010) 040 [arXiv:0910.5670] [INSPIRE].

[6] M. Aganagic, M.C.N. Cheng, R. Dijkgraaf, D. Krefl and C. Vafa, Quantum Geometry of Refined Topological Strings, JHEP 11 (2012) 019 [arXiv:1105.0630] [INSPIRE]. 
[7] J. Kallen and M. Mariño, Instanton effects and quantum spectral curves, Annales Henri Poincaré 17 (2016) 1037 [arXiv:1308.6485] [INSPIRE].

[8] M.-x. Huang and X.-f. Wang, Topological Strings and Quantum Spectral Problems, JHEP 09 (2014) 150 [arXiv:1406.6178] [INSPIRE].

[9] M. Mariño and P. Putrov, Exact Results in ABJM Theory from Topological Strings, JHEP 06 (2010) 011 [arXiv: 0912 .3074] [INSPIRE].

[10] N. Drukker, M. Mariño and P. Putrov, From weak to strong coupling in ABJM theory, Commun. Math. Phys. 306 (2011) 511 [arXiv:1007.3837] [INSPIRE].

[11] M. Mariño and P. Putrov, ABJM theory as a Fermi gas, J. Stat. Mech. 1203 (2012) P03001 [arXiv:1110.4066] [INSPIRE].

[12] Y. Hatsuda, S. Moriyama and K. Okuyama, Exact Results on the ABJM Fermi Gas, JHEP 10 (2012) 020 [arXiv: 1207.4283] [INSPIRE].

[13] Y. Hatsuda, S. Moriyama and K. Okuyama, Instanton Effects in ABJM Theory from Fermi Gas Approach, JHEP 01 (2013) 158 [arXiv:1211.1251] [InSPIRE].

[14] Y. Hatsuda, S. Moriyama and K. Okuyama, Instanton Bound States in ABJM Theory, JHEP 05 (2013) 054 [arXiv: 1301.5184] [INSPIRE].

[15] Y. Hatsuda, M. Mariño, S. Moriyama and K. Okuyama, Non-perturbative effects and the refined topological string, JHEP 09 (2014) 168 [arXiv:1306.1734] [INSPIRE].

[16] Y. Hatsuda, S. Moriyama and K. Okuyama, Exact instanton expansion of the ABJM partition function, PTEP 2015 (2015) 11B104 [arXiv:1507.01678] [INSPIRE].

[17] M. Mariño, Localization at large- $N$ in Chern-Simons-matter theories, arXiv:1608.02959 [INSPIRE].

[18] R. Kashaev and M. Mariño, Operators from mirror curves and the quantum dilogarithm, Commun. Math. Phys. 346 (2016) 967 [arXiv:1501.01014] [InSPIRE].

[19] M. Mariño and S. Zakany, Matrix models from operators and topological strings, Annales Henri Poincaré 17 (2016) 1075 [arXiv: 1502.02958] [INSPIRE].

[20] R. Kashaev, M. Mariño and S. Zakany, Matrix models from operators and topological strings, 2, Annales Henri Poincaré 17 (2016) 2741 [arXiv: 1505. 02243] [INSPIRE].

[21] J. Gu, A. Klemm, M. Mariño and J. Reuter, Exact solutions to quantum spectral curves by topological string theory, JHEP 10 (2015) 025 [arXiv: 1506.09176] [INSPIRE].

[22] K. Okuyama and S. Zakany, TBA-like integral equations from quantized mirror curves, JHEP 03 (2016) 101 [arXiv: 1512.06904] [INSPIRE].

[23] A. Laptev, L. Schimmer and L.A. Takhtajan, Weyl type asymptotics and bounds for the eigenvalues of functional-difference operators for mirror curves, arXiv:1510.00045 [INSPIRE].

[24] G. Bonelli, A. Grassi and A. Tanzini, Seiberg-Witten theory as a Fermi gas, Lett. Math. Phys. 107 (2017) 1 [arXiv: 1603.01174] [INSPIRE].

[25] A. Grassi, Spectral determinants and quantum theta functions, J. Phys. A 49 (2016) 505401 [arXiv: 1604.06786] [INSPIRE].

[26] Y. Sugimoto, Geometric transition in the nonperturbative topological string, Phys. Rev. D 94 (2016) 055010 [arXiv:1607.01534] [INSPIRE]. 
[27] S. Codesido, A. Grassi and M. Mariño, Spectral Theory and Mirror Curves of Higher Genus, Annales Henri Poincaré 18 (2017) 559 [arXiv: 1507.02096] [INSPIRE].

[28] Y. Hatsuda and M. Mariño, Exact quantization conditions for the relativistic Toda lattice, JHEP 05 (2016) 133 [arXiv:1511.02860] [INSPIRE].

[29] S.H. Katz, A. Klemm and C. Vafa, Geometric engineering of quantum field theories, Nucl. Phys. B 497 (1997) 173 [hep-th/9609239] [INSPIRE].

[30] A. Iqbal and A.-K. Kashani-Poor, $\mathrm{SU}(N)$ geometries and topological string amplitudes, Adv. Theor. Math. Phys. 10 (2006) 1 [hep-th/0306032] [INSPIRE].

[31] A.B. Goncharov and R. Kenyon, Dimers and cluster integrable systems, arXiv:1107.5588 [INSPIRE].

[32] S. Franco, Y. Hatsuda and M. Mariño, Exact quantization conditions for cluster integrable systems, J. Stat. Mech. 1606 (2016) 063107 [arXiv: 1512.03061] [INSPIRE].

[33] X. Wang, G. Zhang and M.-x. Huang, New Exact Quantization Condition for Toric Calabi-Yau Geometries, Phys. Rev. Lett. 115 (2015) 121601 [arXiv:1505.05360] [INSPIRE].

[34] K. Sun, X. Wang and M.-x. Huang, Exact Quantization Conditions, Toric Calabi-Yau and Nonperturbative Topological String, JHEP 01 (2017) 061 [arXiv: 1606.07330] [INSPIRE].

[35] M.-X. Huang, A. Klemm and M. Poretschkin, Refined stable pair invariants for E-, $M$ - and [p,q]-strings, JHEP 11 (2013) 112 [arXiv:1308.0619] [INSPIRE].

[36] M.-x. Huang, A. Klemm, J. Reuter and M. Schiereck, Quantum geometry of del Pezzo surfaces in the Nekrasov-Shatashvili limit, JHEP 02 (2015) 031 [arXiv:1401.4723] [INSPIRE].

[37] A. Voros, The Zeta Function Of The Quartic Oscillator, Nucl. Phys. B 165 (1980) 209 [INSPIRE].

[38] K. Konishi and G. Paffuti, Quantum Mechanics: A new introduction, Oxford University Press, Oxford U.K. (2009).

[39] M. Reed and B. Simon, Analysis of operators, Academic Press, (1978).

[40] S. Hosono, A. Klemm and S. Theisen, Lectures on mirror symmetry, Lect. Notes Phys. 436 (1994) 235 [hep-th/9403096] [INSPIRE].

[41] T.M. Chiang, A. Klemm, S.-T. Yau and E. Zaslow, Local mirror symmetry: Calculations and interpretations, Adv. Theor. Math. Phys. 3 (1999) 495 [hep-th/9903053] [INSPIRE].

[42] D.A. Cox and S. Katz, Mirror symmetry and algebraic geometry, American Mathematical Society, Providence U.S.A. (1999).

[43] K. Hori et al., Mirror symmetry, Clay Mathematics Monographs, American Mathematical Society, Providence U.S.A. (2003).

[44] M. Aganagic, A. Klemm and C. Vafa, Disk instantons, mirror symmetry and the duality web, Z. Naturforsch. A 57 (2002) 1 [hep-th/0105045] [INSPIRE].

[45] B. Simon, Notes on infinite determinants of Hilbert space operators, Adv. Math. 24 (1977) 244.

[46] A. Klemm, M. Poretschkin, T. Schimannek and M. Westerholt-Raum, Direct Integration for Mirror Curves of Genus Two and an Almost Meromorphic Siegel Modular Form, arXiv: 1502.00557 [INSPIRE]. 
[47] M.-x. Huang, On Gauge Theory and Topological String in Nekrasov-Shatashvili Limit, JHEP 06 (2012) 152 [arXiv: 1205.3652] [INSPIRE].

[48] M. Bershadsky, S. Cecotti, H. Ooguri and C. Vafa, Kodaira-Spencer theory of gravity and exact results for quantum string amplitudes, Commun. Math. Phys. 165 (1994) 311 [hep-th/9309140] [INSPIRE].

[49] M.-x. Huang and A. Klemm, Direct integration for general $\Omega$ backgrounds, Adv. Theor. Math. Phys. 16 (2012) 805 [arXiv: 1009.1126] [INSPIRE].

[50] D. Krefl and J. Walcher, Extended Holomorphic Anomaly in Gauge Theory, Lett. Math. Phys. 95 (2011) 67 [arXiv: 1007.0263] [INSPIRE].

[51] R. Gopakumar and C. Vafa, M theory and topological strings. 2., hep-th/9812127 [INSPIRE].

[52] A. Iqbal, C. Kozcaz and C. Vafa, The refined topological vertex, JHEP 10 (2009) 069 [hep-th/0701156] [INSPIRE].

[53] J. Choi, S. Katz and A. Klemm, The refined BPS index from stable pair invariants, Commun. Math. Phys. 328 (2014) 903 [arXiv:1210.4403] [InSPIRE].

[54] N. Nekrasov and A. Okounkov, Membranes and Sheaves, arXiv:1404.2323 [INSPIRE].

[55] M. Aganagic, V. Bouchard and A. Klemm, Topological Strings and (Almost) Modular Forms, Commun. Math. Phys. 277 (2008) 771 [hep-th/0607100] [INSPIRE].

[56] S. Garoufalidis and R. Kashaev, Evaluation of state integrals at rational points, Commun. Num. Theor. Phys. 09 (2015) 549 [arXiv:1411.6062] [INSPIRE].

[57] C.A. Tracy and H. Widom, Proofs of two conjectures related to the thermodynamic Bethe ansatz, Commun. Math. Phys. 179 (1996) 667 [solv-int/9509003] [INSPIRE].

[58] P. Putrov and M. Yamazaki, Exact ABJM Partition Function from TBA, Mod. Phys. Lett. A 27 (2012) 1250200 [arXiv:1207.5066] [InSPIRE].

[59] L.D. Faddeev and R.M. Kashaev, Quantum Dilogarithm, Mod. Phys. Lett. A 9 (1994) 427 [hep-th/9310070] [INSPIRE].

[60] L.D. Faddeev, Current-like variables in massive and massless integrable models, hep-th/9408041 [INSPIRE].

[61] L.D. Faddeev, Discrete Heisenberg-Weyl group and modular group, Lett. Math. Phys. 34 (1995) 249 [hep-th/9504111] [INSPIRE].

[62] A. Brini and A. Tanzini, Exact results for topological strings on resolved $Y^{* *} p, q$ singularities, Commun. Math. Phys. 289 (2009) 205 [arXiv:0804.2598] [InSPIRE].

[63] F. Rodriguez Villegas, Modular Mahler measures, I, in Topics in number theory, Kluwer Acad. Publ., Dordrecht, The Netherlands (1999), p. 17.

[64] C. Doran and M. Kerr, Algebraic K-theory of toric hypersurfaces, Commun. Number Theory Phys. 5 (2011) 397 [arXiv:0809.4669].

[65] R. Eager, S. Franco and K. Schaeffer, Dimer Models and Integrable Systems, JHEP 06 (2012) 106 [arXiv:1107.1244] [INSPIRE].

[66] A. Marshakov, Lie Groups, Cluster Variables and Integrable Systems, J. Geom. Phys. 67 (2013) 16 [arXiv:1207.1869] [INSPIRE].

[67] V.V. Fock and A. Marshakov, Loop groups, Clusters, Dimers and Integrable systems, arXiv: 1401.1606 [INSPIRE]. 
[68] M. Mariño and S. Zakany, Exact eigenfunctions and the open topological string, arXiv: 1606.05297 [INSPIRE].

[69] A.-K. Kashani-Poor, Quantization condition from exact WKB for difference equations, JHEP 06 (2016) 180 [arXiv:1604.01690] [INSPIRE].

[70] A. Sciarappa, Bethe/Gauge correspondence in odd dimension: modular double, non-perturbative corrections and open topological strings, JHEP 10 (2016) 014 [arXiv: 1606.01000] [INSPIRE].

[71] H. Sakai, Rational surfaces associated with affine root systems and geometry of the Painlevé equations, Commun. Math. Phys. 220 (2001) 165.

[72] A. Grassi and J. Gu, BPS relations from spectral problems and blowup equations, arXiv: 1609.05914 [INSPIRE]. 